RAND

conrogarion

\title{
Evaluation of the Emergency Education Response for Syrian Refugee Children and Host Communities in Jordan
}

Shelly Culbertson, Tom Ling, Marie-Louise Henham, Jennie Corbett, Rita Karam, Paulina Pankowska, Catherine Saunders, Jacopo Bellasio, Ben Baruch

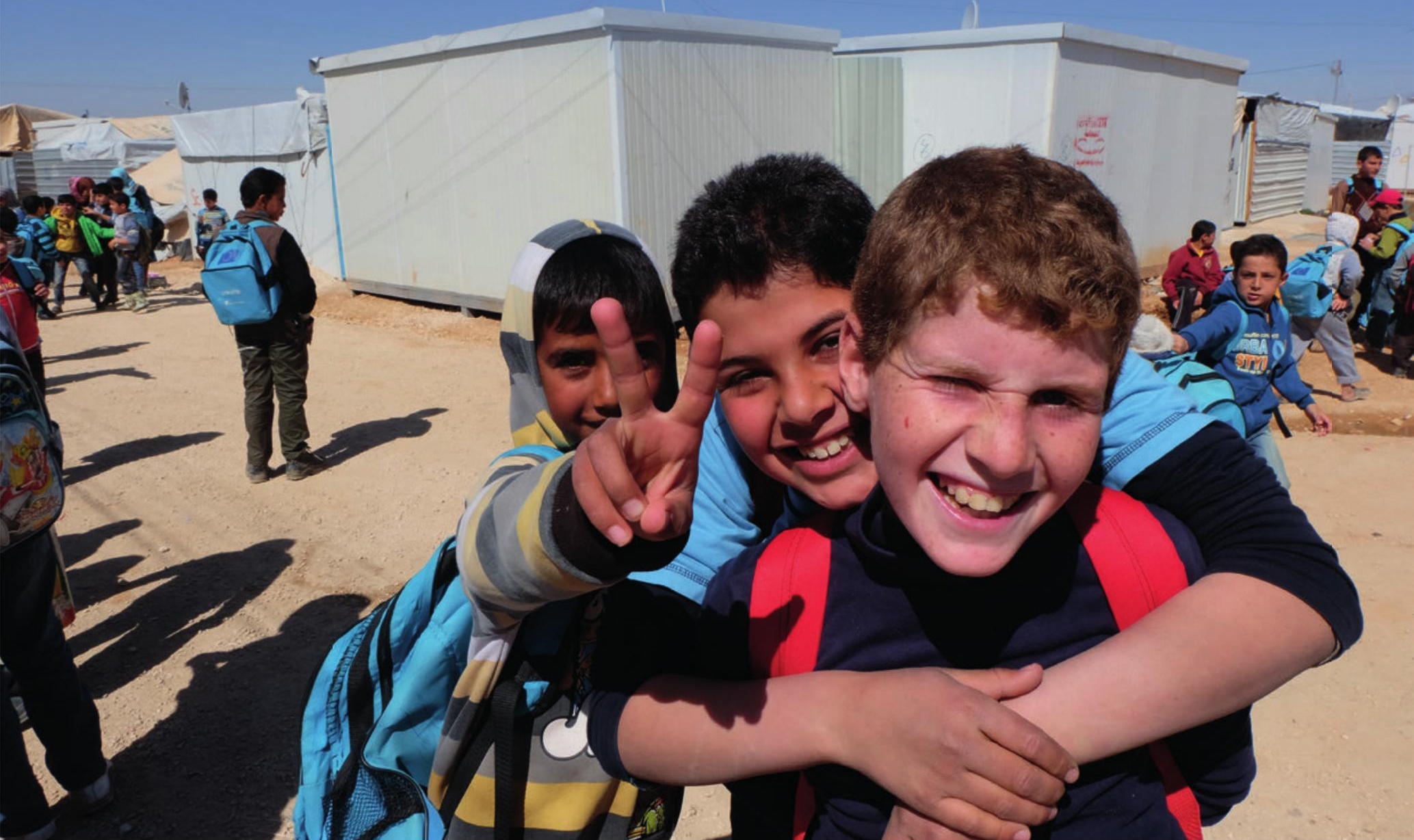


For more information on this publication, visit www.rand.org/t/rr1203

Published by the RAND Corporation, Santa Monica, Calif., and Cambridge, UK

RAND $^{\circledR}$ is a registered trademark.

(C) 2016 UNICEF

Image credits

Photovoice (Chapters 3 and 5): UNICEF, all others: Marie Louise Henham

The RAND Corporation is a research organization that develops solutions to public policy challenges to help make communities throughout the world safer and more secure, healthier and more prosperous. RAND is nonprofit, nonpartisan, and committed to the public interest.

All rights reserved. No part of this book may be reproduced in any form by any electronic or mechanical means (including photocopying, recording, or information storage and retrieval) without permission in writing from the sponsor.

Support RAND

Make a tax-deductible charitable contribution at www.rand.org/giving/contribute 


\section{Preface}

The crisis in Syria has displaced nearly half of Syria's population, with citizens fleeing internally or to neighbouring countries. A key responsibility and challenge for host nations is providing education for the refugees. The 'Emergency Education Response for Displaced Syrian Children and Host Communities in Jordan' programme implemented by UNICEF, the Government of Jordan and their partners, aims to provide Syrian refugee children with access to appropriate, quality education. Contracted by UNICEF, this evaluation, conducted by RAND, reviews the progress achieved in implementing the programme and identifies key successes, effective practices and gaps that need to be addressed.

For more information about UNICEF or this report, contact Michiru Sugi. Sugi can be reached by email at msugi@unicef.org; by phone at +962 65502452 ; or by mail at UNICEF Jordan, Building 15, Abdulqader Al-Abed Street, Tla'a Al-Ali, Amman, Jordan.
The RAND Corporation is a non-profit institution that helps improve policy and decisionmaking through research and analysis. RAND focuses on the issues that matter most, such as education, health, national security, international affairs, law and business, the environment and more. Through research and analysis, RAND helps public and private decisionmakers solve problems, tackle challenges and identify ways to make society safer, smarter and more prosperous. Shelly Culbertson and Tom Ling were the principal investigators of this evaluation. Culbertson can be reached by e-mail at shellyc@rand.org; by phone at +1 412-683-2300 x4666; or by mail at the RAND Corporation, 4570 Fifth Ave, Su-ite 600, Pittsburgh, Pennsylvania 15213, USA. Ling can be reached by email at tling@rand.org; by phone at +44(0)1223 222744; or by mail at RAND Europe, Westbrook Centre, Milton Road, Cambridge CB4 1YG, UK. 

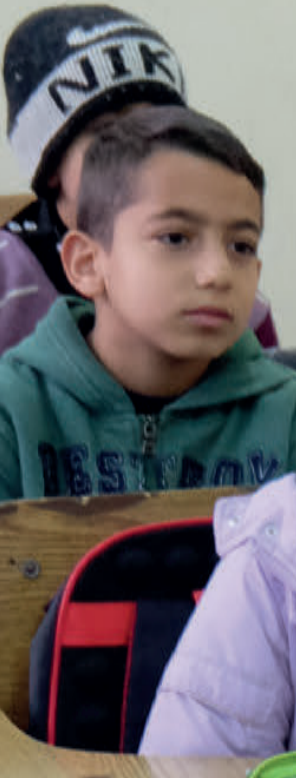

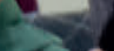

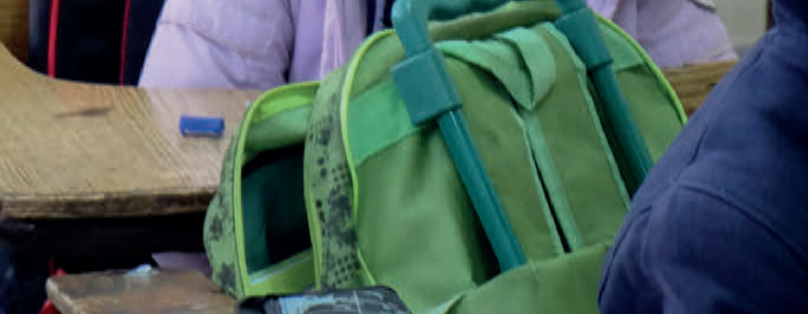

$\frac{1}{3}$

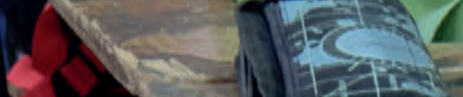
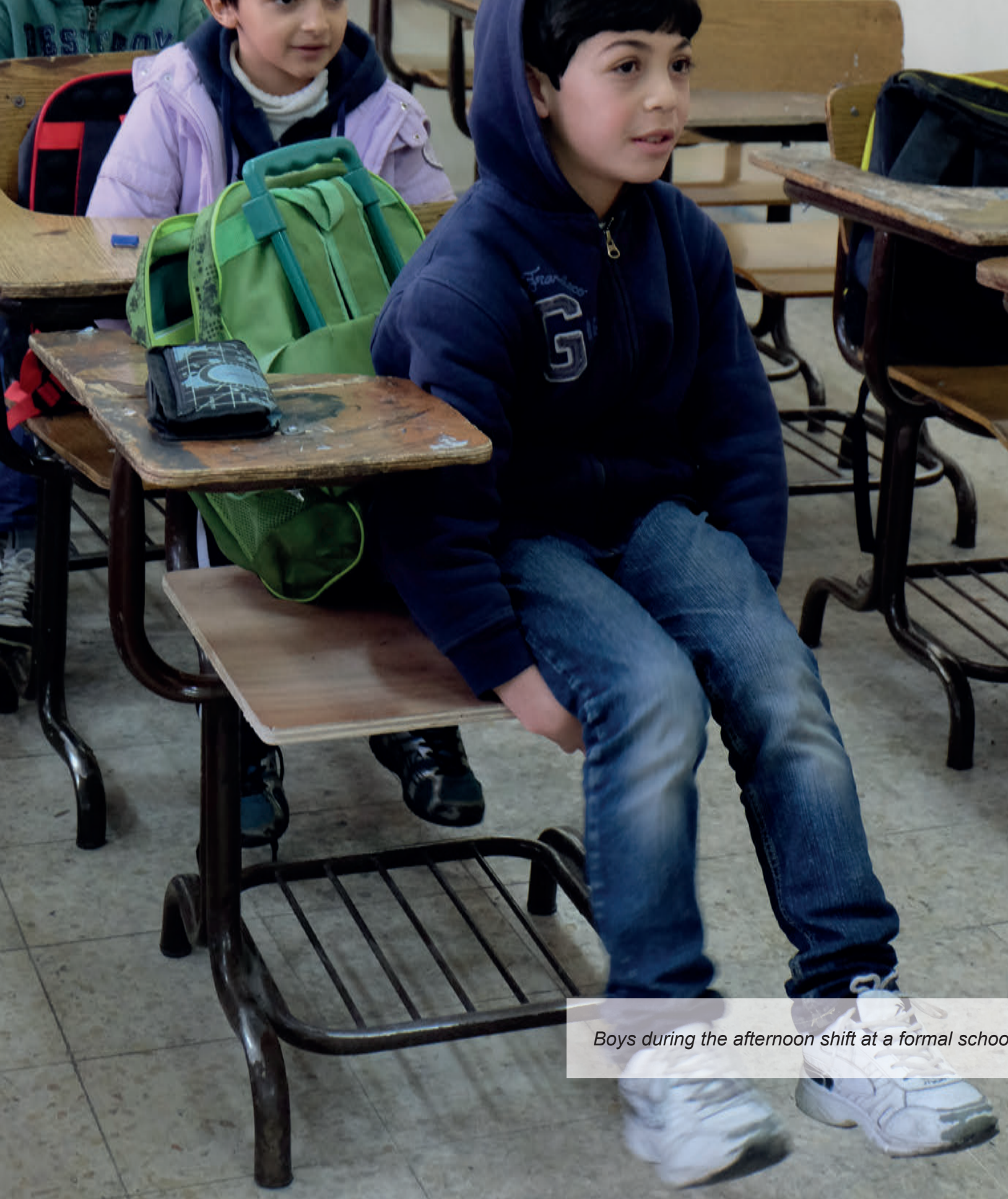

12
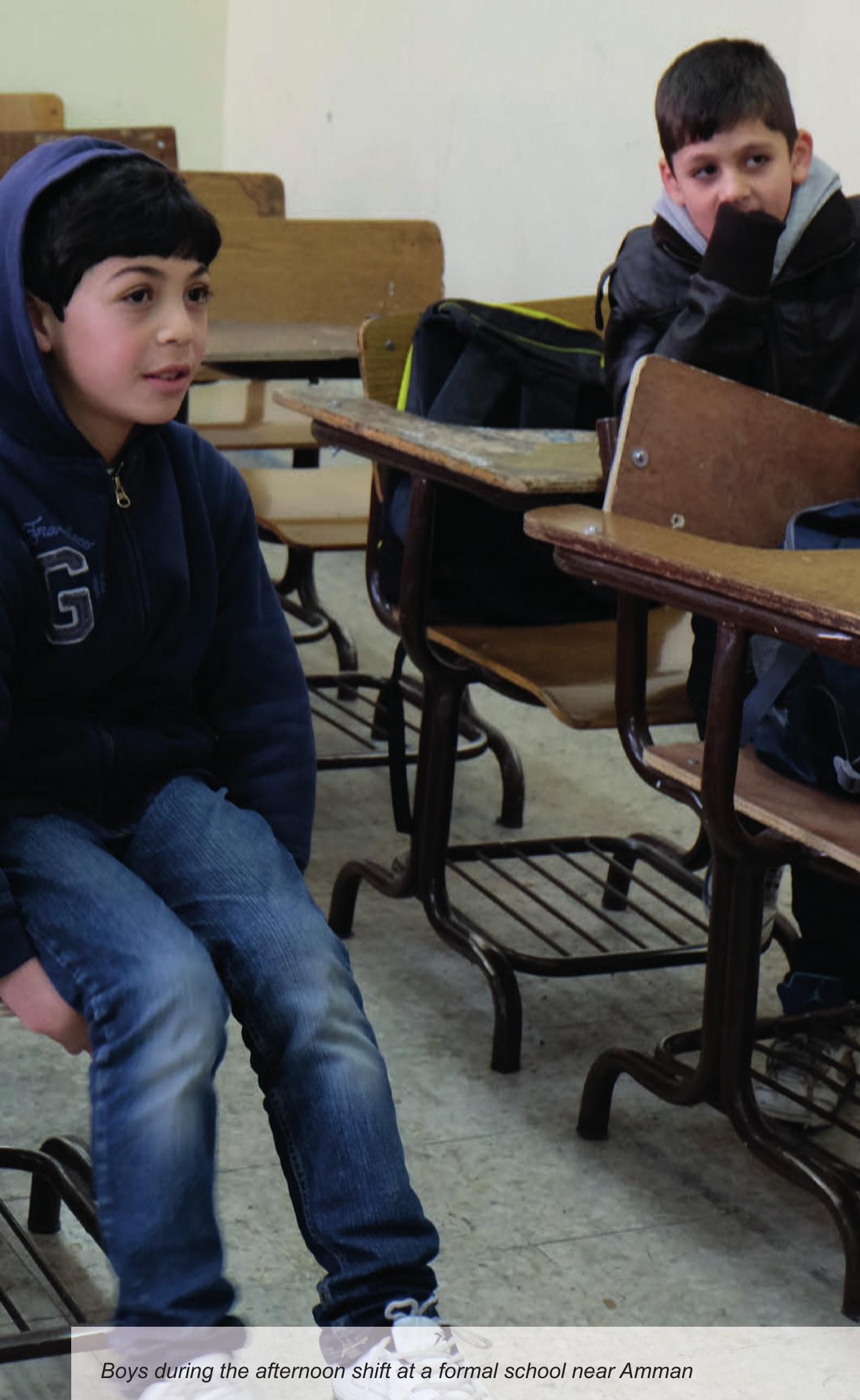


\section{Table of contents}

Preface $\quad$ i

Table of contents iii

Figures and tables $v$

Executive summary vii

Acknowledgements $\quad$ xiii

Abbreviations $\quad$ xv

$\begin{array}{ll}\text { Chapter One. Introduction, background and approach } & 1\end{array}$

1.1. Background to the Emergency Education Response Programme 1

1.2. Evaluation purpose and objectives 4

1.3. Evaluation scope and focus 4

1.4. Methodology and limitations $\quad 6$

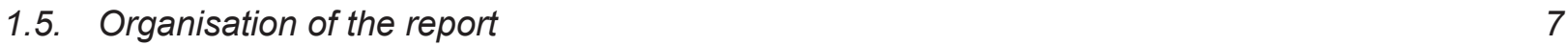

$\begin{array}{ll}\text { Chapter Two. Findings: relevance } & \mathbf{9}\end{array}$

2.1. The EER lacks an explicit theory of change, limiting adaptation to a changing strategic context

2.2. There are significant challenges to the EER being and remaining relevant 11

2.3. Developing a theory of change 14

2.4. Summary and conclusions 15

$\begin{array}{ll}\text { Chapter Three. Findings: effectiveness } & 17\end{array}$

3.1. Access to formal education 17

3.2. Quality of formal education $\quad 27$

3.3. Access, quality and strategy of alternative education 34

3.4. Summary and conclusions 39

$\begin{array}{ll}\text { Chapter Four. Findings: efficiency } & \mathbf{4 1}\end{array}$

4.1. Identify needs and efficient delivery mechanisms 43

4.2. Justifying budgets and securing funding 46

4.3. Coordinating and aligning for maximum efficiency 46

4.4. Deliver value for money and manage for results 49

4.5. Monitor, evaluate and learn to improve and build capacity 51

4.6. Summary and conclusions $\quad 52$

Chapter Five. Findings: cross-cutting issues $\quad \mathbf{5 5}$

5.1. Coordination and cross-sectoral cooperation $\quad 55$

5.2. Gendered differences and approaches $\quad 57$

5.3. Children with special needs and disabilities 61

5.4. Health and nutrition 63 
5.5. Child protection $\quad 65$

5.6. WASH

$\begin{array}{ll}\text { 5.7. Summary and conclusions } & 70\end{array}$

$\begin{array}{ll}\text { Chapter Six. Findings: sustainability } & 73\end{array}$

6.1. Making trade-offs $\quad 73$

6.2. Advocacy and policy dialogue $\quad 75$

6.3. Capacity building 76

6.4. Funding

6.5. Community engagement and empowerment 77

$\begin{array}{ll}\text { 6.6. Summary and conclusions } & 78\end{array}$

Chapter Seven. Recommendations $\quad 79$

7.1. Build upon significant accomplishments $\quad 79$

7.2. Develop a clearly articulated medium-term (5 to 10-year) vision 80

7.3. Develop a medium-term outlook to funding 80

7.4. Prioritise expansion of formal education and creation of school spaces through investing in school infrastructure, teacher salaries and supportive planning 81

7.5. Create formal education at the scale needed for Syrian and Jordanian children who have missed years of school 83

7.6. Create a new delivery model to support short-term rapid expansion of education while building long-term sustainable options for out-of-school children 83

7.7. Invest in MOE capacity and provide direct operational support to the MOE 85

7.8. Improve the performance of double-shifted schools to meet the specific needs of both Jordanians and Syrians

7.9. Improve the quality and safety of the learning environment in MoE formal schools in camps and host communities

7.10. Improve access and quality by addressing social challenges and targeting the different needs of girls and boys

7.11. Prioritise programmes and spending based on options analyses 91

7.12. Strengthen sectoral coordination and management 92

7.13. Ensure flexibility and adaptability as circumstances change 93

7.14. Develop a phased implementation plan with 'quick wins' 93

$\begin{array}{ll}\text { References } & 95\end{array}$

$\begin{array}{ll}\text { Annex 1: Evaluation questions and summary findings } & 103\end{array}$

Annex 2: Further detail on methodology 107

Sampling and geographical coverage $\quad 107$

$\begin{array}{ll}\text { Preparatory work for participatory photography } & 108\end{array}$

Limitations to the focus group and survey data analysis methodologies 108

Ethical issues 109

$\begin{array}{ll}\text { Human rights and gender equality } & 109\end{array}$

Annex 3: Summaries of challenges, effective practices and remaining needs in key areas of the evaluation

Annex 4: Comparison (where possible) of budgeting requirements between 3RP and RRP6 


\section{Figures and tables}

Figure 1.1: Components of the education response, evaluation criteria and questions, and data sources

Figure 2.1: The proposed Emergency Education Response ToC for this evaluation

Figure 3.1: Attendance rates to formal education (among Syrian refugee children 6-17 years old) in host communities and Zaatari Camp

Figure 3.2: Variation in attendance of Syrian refugee children in formal education by district in Zaatari Camp and by governorate in host communities

Figure 3.3: Enrolment of Syrian girls and boys in host communities and Zaatari Camp

Figure 4.1: The EER cycle of delivering efficient services

Figure 5.1: Enrolment rates of children with and without disabilities in Zaatari and host communities

Figure 6.1: Factors Affecting Sustainability of the Emergency Education Response

Figure 6.2: Trade-offs affecting sustainability

Table 2.1: Relevance - overview of evaluation questions and findings

Table 3.1: Effectiveness - overview of evaluation questions and findings

Table 3.2: Syrian refugee children attending types of education and out of school in 2014-2015

Table 3.3: Factors associated with children's access to education

Table 3.4: Double-shifted schools for Syrians by location

Table 4.1: Efficiency - overview of evaluation questions and findings

Table 4.2: Summary comparison (where possible) of budgeting requirements between 3RP (2015) and RRP6 (2014, January to June only)

Table 4.3: Cost per child of key alternative education activities interventions 2014-2015 (US\$)

Table 4.4: Comparison of estimates of instructional time per programme

Table 5.1: Cross-cutting - overview of evaluation questions and findings 56

Table 6.1: Sustainability - overview of evaluation questions and findings 74

Table A2.1: Methods, sampling and geographical coverage of the evaluation 
Table A3.1: Summary table - relevance

Table A3.2: Summary table - effective provision of access to formal education opportunities

Table A3.3: Summary table - effective approaches to improve the quality of formal education provision

Table A3.4: Summary table - addressing the needs of children excluded from formal education opportunities accessing alternative learning activities in community and camp settings

Table A3.5: Summary table - efficiency

Table A3.6: Summary table - gendered differences and approaches

Table A3.7: Summary table - children with special needs and disabilities

Table A3.8: Summary table - health and nutrition

Table A3.9: Summary table - child protection and psychosocial support

Table A3.10: Summary table - WASH

Table A3.11: Summary table - sustainability

Table A3.12: Summary table - recommendations 


\section{Executive summary}

This evaluation reviews the Emergency Education Response (EER) programme in Jordan of 2012-2014, with consideration of events in early 2015 . It provides an analysis of the EER, and the contribution made by stakeholders including the Government of Jordan (GOJ), UNICEF, donors, implementing partners and other UN agencies, with particular attention to the role of UNICEF. The purpose of this evaluation is to provide reliable evidence on the EER to support judgements about its performance, describe examples of good practice and lessons learned, and offer recommendations to guide future actions.

Specifically, the evaluation assesses the EER's performance in providing formal education in both camps and host communities, alternative programmes, supportive programmes and systems, and the minimisation of impact on the Jordanian host community. It does this using the OECD DAC evaluation criteria ${ }^{1}$ of programme relevance, effectiveness, efficiency and sustainability, as well as specific evaluation questions developed for this study. The work is further shaped by recognising the importance of each child's rights, while being sensitive to the realities of an emergency response with limited resources in a challenging context.

The evaluation relies on both quantitative and qualitative methods of data collection and analysis including: a document and literature review; stakeholder interviews (with the Government of Jordan, UN agencies, donors, NGOs, municipal and camp education authorities, and school principals); school visits; focus groups with children, parents, and teachers; and RAND's secondary analysis of Zaatari and host community Joint Education Needs Assessment (JENA) survey data.

\section{Overview of the Emergency Education Response}

The EER aims to contribute safe and appropriate services for Syrian refugees in Jordan and to ensure free formal education and additional relevant education services for Syrian refugee children. ${ }^{2}$ In 2012, the Jordanian Government made a decision to provide education through the formal Ministry of Education system to Syrian refugee children in both host communities and camps. UNICEF provided planning, implementation, technical support, capacity building, and monitoring and evaluation. UNICEF is also working with its partners in refugee camps and host communities to create and develop alternative education opportunities for children. NGO partners provide implementation and technical expertise. Donors, including the United States, the EU and others, provide funding. The achievements of the EER are considerable and include: structured formal education for 130,000 children, informal and non-formal education for 35,000 children; 69 schools with furniture and equipment and 65 schools with prefab classrooms; 98 additional double-shifted schools that expanded access; training for 2,100 teachers; outreach and information services; measures to target vulnerable children (such as psychosocial support); mobilising significant resources for the education response; and engaging the skills of stakeholders including the Government of Jordan, UNICEF and NGOs. 


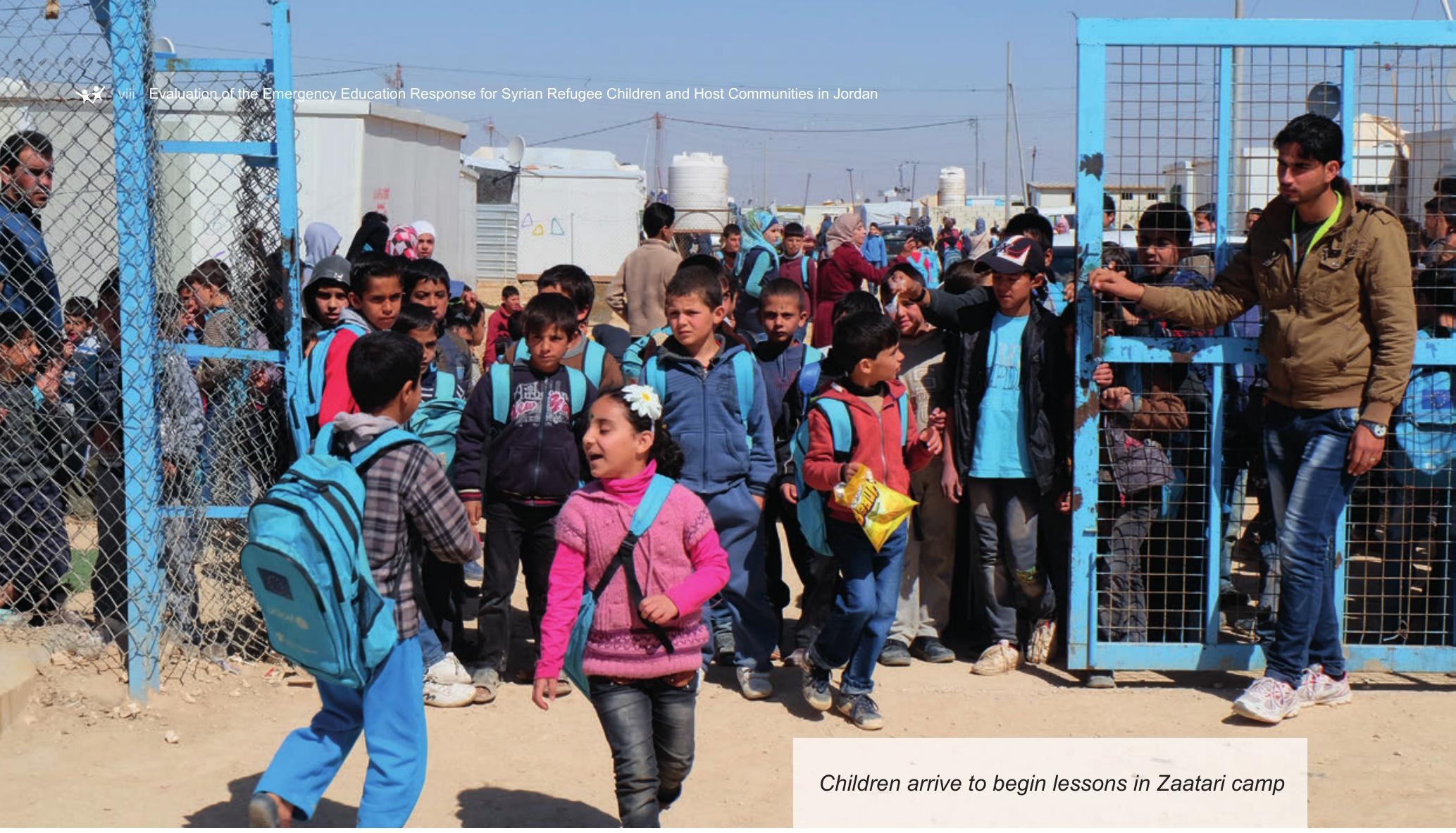

However, substantial problems have yet to be resolved. Some 40 per cent of children to not have access to formal education (see data in Chapter 3); there is widespread overcrowding in both host community and camp schools; bullying is widespread; teachers struggle with the challenges they face in the classroom; and few are trained in dealing with the psychosocial problems of many of the pupils. In double-shifted schools, instructional time is shortened and amenities are poor.

The partners delivering education for these children are progressively identifying and responding to not only issues of access but also issues of quality; as the crisis becomes longer term, a short-term response to an immediate crisis is developing into an effort to build resilience and sustainability.

\section{Was the EER relevant?}

The first responses to the Syrian refugee crisis in 2012-2013 were relevant to immediate education needs, delivering a rapid response that enabled many children to enrol in school. However, as the Syrian crisis continued, the EER, and UNICEF in particular, had to adapt.
With the transition towards providing education in the medium-term, strategic efforts and planning lagged behind events on the ground. High level documents (RRP6, 3RP, NRP and JRP) describe objectives, needs and activities, but there is no single document that outlines the EER's strategy or Theory of Change. Creating a relevant strategic response has faced challenging factors including: complex, interacting components in managing the education response; rapidly changing circumstances and growth in numbers of refugees; lack of consensus on the time frame of the crisis; patchy transition from emergency response to resilience; disparate agendas, values and goals among stakeholders; lack of strategic and budget prioritisation for longer-term systems strengthening; the need for new skills and approaches to a largely urban crisis in a middle-income country; and tensions between a nationality-based approach and a vulnerability or rights-based approach.

\section{Was the EER effective?}

Our analysis of effectiveness considered the goals of the EER as articulated in the RRP6 and 3RP: access to education, quality of education and meeting the needs of vulnerable Jordanians. 
Providing access to formal education for 130,000 Syrian children is a significant accomplishment of the EER. However, having at least 97,000 Syrian children out of formal school requires urgent action. Improving access depends on understanding determinants of access that include location, characteristics of education provision, and family and child characteristics. Factors related to location and educational provision can be addressed through educational policy. Factors related to the family and child may require a multi-sectoral approach with key actors collaborating to prioritise and develop solutions.

Quality of education provided to Syrian refugees has been a secondary consideration to access. It has been below desired levels, with quality challenges also affecting Jordanians. There has been limited use of quality measures: test scores are low; classrooms are crowded; teachers find environments challenging; facilities are poorly maintained; Syrian students struggle with transitioning to Jordanian curricula; grade placement policies are inconsistent; and unclear pathways to adulthood demotivate students. In some ways, double-shifting has provided opportunities to expand access and meet the quality needs of both Syrians and Jordanians, but the double shifts in Jordan (now 15 per cent of Jordan's education provision) work worse than elsewhere as they offer decreased instructional time and do not provide equal resources to both shifts. Additional influxes of students into Jordan's formal system have crowded out resources for quality improvements for Jordanians, and classrooms themselves have become more crowded.

Alternative education has been provided for 35,000 Syrian refugee children, and providing such access has been more effective in camps than in host communities. Alternative provision is perceived to provide high-quality, relevant, child-centred and flexible education to Syrians. However, there is a lack of a clear framework for pathways between alternative education and formal education.

\section{Was the EER efficient?}

This evaluation identified good examples of stepping towards greater efficiency. There are improved data for strategic decisionmaking, and examples of learning and adaptation (for example, Makani centres). There is a base upon which to build, which includes good macro-level data (especially in camps), useful project-level data, and partners who know and trust each other. Data have been 'pulled through' into decisionmaking. There have also been improving data on implementing partners' activities, and examples of learning and adaptation. In particular, the Government of Jordan continues to support the EER by providing education resources. UNICEF has played a key role in improving the efficiency of the EER by ensuring that the distribution of needs is accurately analysed, a balanced portfolio of appropriate activities is established, an outcomes-focused management approach is adopted, and learning is supported by monitoring and evaluation. The EER has ensured the availability of information about the nature and distribution of needs, and such data were often relevant, sufficient and credible.

However, there are several areas where efficiency could be improved. The evaluation found little evidence that options are fully appraised, with optimal approaches adopted in the face of resource constraints. As a planning and fund-raising tool, key planning documents (e.g. RRP6 and 3RP) are limited by cynicism regarding the amounts of funding requested, the view that it was not prioritised, and the perceived lack of transparency for donors. For some stakeholders, coordination between UNICEF and the Government of Jordan has been characterised by grey areas of accountability related to UNICEF's changing role in the response over time and ownership of education services in camps. Based on the available data, a cost effectiveness analysis is not possible. Developing this evidence base should be a focus in the future; many of the components required to deliver value for money are either in 
place or being developed, and these should be communicated across the EER within an overall strategy. In general, feedback and learning mechanisms are weak or under-resourced and use of outcome data is limited. Further donor confidence would be achieved by sharpening the focus of monitoring towards delivering outcomes.

\section{How effective were cross-cutting activities?}

A range of cross-cutting issues interact with education, and impact upon access, quality and delivery of services under the EER. These issues include gender and children with special needs and disabilities. Cross-cutting sectors include health and nutrition, child protection (including psychosocial support) and water, sanitation and hygiene (WASH).

Efforts around gender mainstreaming and capacity building, enhanced coordination and disaggregated reporting, show that gender is an EER priority. However, crucial gaps remain: programmatic focus is still overwhelmingly on outreach and support for girls, undervaluing the severe challenges boys face, such as high rates of child labour and low classroom engagement. A lack of adequate psychosocial support in schools and unsafe school environments, with bullying as a particular problem, mean that some children face severe risks. Effective practice initiatives such as Makani centres show promise; however, strategic action is needed to address gaps in the protective learning environment of $\mathrm{MOE}$ schools. Efforts to build government capacity around WASH in schools and develop genderappropriate facilities and infrastructure have had significant reach, but infrastructure problems persist in some schools and in others WASH facilities fall short in terms of cleanliness. Health interventions and assessments in schools have reached many children. However, enhanced coordination is needed as access to healthcare remains a challenge and some children go to school hungry. Furthermore, while cross-sectoral collaboration has strengthened significantly over time thanks to investment in coordination structures and key initiatives, increased investment is needed to address gaps in operational synergies. While Programme efforts have been impressive, with so many children out of school and in compromised learning environments, the EER is falling short of fulfilling UNICEF's Core Commitments for Children in Humanitarian Action, a framework based on international human rights and humanitarian laws and standards.

\section{Is the EER sustainable?}

Now that it is clear that the EER will have a continuing role over the medium term, the question of its sustainability is more urgent. It is likely that many Syrian refugees will be in Jordan for at least the next decade, perhaps longer. It is also accepted that international funding may not be sufficient to meet all needs; aid may decline over time, and programmes may increasingly rely on local actors (the Government of Jordan, Jordanian civil society and Syrians themselves). This future needs to be planned for.

Sustainability involves: making trade-offs and priorities where objectives outstrip the capacity to deliver them; advocacy, policy dialogue and coordination; building capacity in national entities; creating longer-term funding mechanisms; and engaging the communities. Making trade-offs has been constrained by the different values and priorities of stakeholders. While there are significant coordination structures, there are missed opportunities for donors to pool advocacy. Funding has been on short-term cycles. Capacity building has started, but there is a further need to build MOE management capability, sustainably pay teachers, build infrastructure and expand MOE quality assurance programmes for schools. While the inclusion of Syrian and Jordanian community voices was not prioritised from the outset, interviews reflect stakeholders' acknowledgement of its importance going forward, for both community cohesion and 
not enough school spaces in Jordan, and over 500 schools are already double-shifted to meet Jordanian and Syrian needs. Options to scale up education provision rapidly include: more double shifts; prefabricated schools; renting and repurposing other buildings; and using school buses. In the longer term, donors and the GOJ should invest in school buildings. The provision of teacher salaries by donors to expand formal education is also a priority. This also requires creating formal education at scale for children who have missed years of school. To support the short-term rapid expansion of education while building long-term sustainability for out-of-school children, there should be a new delivery model. This will involve UN agencies and partners supporting the development of programmes, with a planned transition to the MOE taking a greater role. This model will balance the urgent need to scale up education with the promotion of sustainability. At the same time, there is a need to invest in MOE capacity. Sustainability requires the strengthening of Jordanian institutions.

Next, there are ways to improve the performance of double-shifted schools to meet the needs of both Jordanians and Syrians. In particular, these include increasing the number of school days to accommodate for reduced teaching time in shifts and keeping resources in both shifts equivalent. To promote quality, fairness and coping for both Syrians and Jordanians, this evaluation provides criteria for when to use integrated classrooms, separated classrooms, single shifts and double shifts.

Steps also need to be taken to improve the quality and safety of school learning environments. Understandably, quality of education has taken lower priority than access to education during the emergency's early stages. However, now planning should shift towards quality. Options emerging from this evaluation focus on reducing class sizes, school monitoring, mixing experienced and inexperienced teachers, paying teachers adequately, hiring Syrian teachers, training, developing pathways between education and adulthood, creating additional structured psychosocial support in both camps and host communities, addressing bullying, engaging parents, and investing in the classroom environment.

Girls and boys face different gendered challenges both in the classroom and outside, and responses need to be targeted to different gendered needs. Boys have lower access to formal education at all levels. For girls, access to education is affected by early marriage, concerns about safety in transportation and sexual and gender-based violence. Much of this is related to wider societal issues, as opposed to education policy. An approach that the education community, in collaboration with the GOJ, can take is advocacy with stakeholders in other sectors in order to improve upon these circumstances.

To address these multiple priorities, there is a need to prioritise programmes and spending based on options analyses. Steps should be taken to strengthen management, such as developing standardised cost and performance data. Given uncertainty about the future needs and circumstances of Syrian refugees in Jordan, planning needs to explicitly recognise key unknowns and be phased. Scenario planning can be used to create plans relevant in multiple future scenarios when there are significant uncertainties, but also strongly held values and commitments. A maturity model addresses where programmes are now, where they could be, and the steps that would move them towards excellence. An implementation plan, with emphasis on early 'quick wins' should be developed. 


\section{Acknowledgements}

The authors would like to thank UNICEF for sponsoring this evaluation and would especially like to acknowledge the continued support of Susan Ayari, Farhod Khamidov, Michiru Sugi, Randa Nubani, Farrukh Mirza and Faizah Samat from UNICEF.

Furthermore, the authors would like to thank Muna Haddad, Raghda Quandour, Sirsa Qursha and Zina Khoury for conducting focus groups with teachers, parents and children. Merissa Khurma also provided the team with support and assistance when conducting stakeholder interviews in Jordan, and the team would like to extend their thanks to her. The team would also like to thank the NGO partners who helped the evaluation team to set up focus groups (the Islamic Charity Center Society, the Norwegian Refugee Council, Mercy Corps, Questscope Relief International and Save the Children Jordan) and all the teachers, parents and children who took the time to attend and contribute to the focus group discussions.
In addition, the authors would like to thank the following stakeholders for participation in interviews, sharing documents and data, and contribution of ideas: Jordan's Ministry of Education, Jordan's Ministry of Planning and International Cooperation, UNICEF, UNESCO, UNHCR, Canadian Development Aid, the EU delegation to Jordan the US Department of State's Bureau of Population, Refugees, and Migration (BPRM), the Islamic Charity Center Society, Madrasti, MECI Institute, Mercy Corps, the Norwegian Refugee Council, Relief International, Save the Children Jordan, Yarmouk Baqaa Club and the teachers and principals of schools interviewed in Amman and Zarqa.

Finally, we would like to thank $\mathrm{Dr}$ Christian van Stolk and Alexandra Pollitt for their RAND Quality Assurance review of the report. 



\section{Abbreviations}

Bureau of Population, Refugees, and Migration

BPRM

Core Commitments for Children

CCC

Division for Programme Support and Management

DPSM

Education Management Information System

EMIS

Education Reform for Knowledge Economy Project

Erfke

Evaluation Reference Group

ERG

Education Sector Working Group

ESWG

Finn Church Aid

FCA

Government of Jordan

GOJ

Human Subjects Protection Committee

HSPC

Implementing Partners

IPs

Institute for Family Health

IFH

Inter-Agency Task Force

IATF

Inter-Agency Working Group on Children's Participation

IAWGCP

Inter-Sector Working Group

ISWG

Joint Education Needs Assessment

JENA

Millennium Development Goals Report

MDGR

Ministry of Education

MOE

Ministry of Planning and International Cooperation

MOPIC

Monitoring and Evaluation

M\&E

Monitoring Results for Equity System

MORES

Non-Food Items

$\mathrm{NFI}$

Non-Governmental Organisation

NGO

Noor Al-Hussein Foundation

NHF

Norwegian Refugee Council

NRC

National Resilience Plan

NRP 
Organisation for Economic Co-operation and Development

Operational Solutions and Transition Section

OSTS

Out-Of-School Children

OOSC

Programme Cooperation Agreements

PCAs

Research Ethics Advisory Group

REAG

Regional Refugee And Resilience Plan

3RP

Regional Response Plan 6

RRP6

Sexual and Gender-Based Violence

SGBV

Trends In International Mathematics and Science Study

TIMSS

Theory of Change

$\mathrm{ToC}$

United Nations

UN

United Nations High Commissioner for Refugees

UNHCR

United Nations Children's Fund

UNICEF

United Nations Educational, Scientific and Cultural Organization

UNESCO

Water, Sanitation and Hygiene

WASH 


\section{Introduction, background and approach}

Since the start of the crisis in Syria in the spring of 2011, nearly half of Syria's population of 23 million has been displaced, either internally or as refugees. Over 3.9 million Syrians have fled Syria to escape violence in their country. ${ }^{3}$ As of May 2015, 630,000 Syrian refugees have registered with the UN High Commissioner for Refugees (UNHCR) in Jordan, ${ }^{4}$ representing a 10 per cent increase in Jordan's population since 2011. The Government of Jordan (GOJ) estimates that actual numbers may be higher, with up to 1.4 million Syrians in Jordan, if unregistered refugees are considered. ${ }^{5}$ The flow of people has not stopped. Throughout this humanitarian crisis, Jordan has demonstrated generosity by keeping its borders open and by extending services to Syrian refugees. Jordan's national government and municipal governments, Jordanian citizens, UN agencies, national and international NGOs and foreign donors have all contributed towards stemming the crisis.

In response to increasing educational needs, UNICEF, the GOJ and multiple partners launched the Emergency Education Response (EER) Programme in April 2012. ${ }^{6}$ A significant accomplishment of the Programme is the provision of formal education to 130,000 Syrian children and alternative education to 35,000

Syrian children (out of the 210,000 Syrian school-age children in Jordan). However, important needs remain in providing access to education to the Syrians, improving quality and increasing efficiency. Furthermore, the substantial numbers of refugees are perceived to have had adverse consequences on the Jordanian education system, with particular impact born by vulnerable Jordanians.

To understand the achievements to date, assess lessons learned, describe remaining needs and advise on future steps in providing education to the Syrian refugees, this evaluation of the EER Programme was commissioned by UNICEF.

\subsection{Background to the Emergency Education Response Programme}

The EER Programme aims to contribute safe and appropriate services for vulnerable Syrian refugees living in Jordan and to ensure free formal education and additional relevant education services are available to Syrian refugee children. ${ }^{7}$ In 2012, the GOJ made a decision to provide education through the formal Ministry of Education (MOE) system to Syrian refugee children in both host communities and 


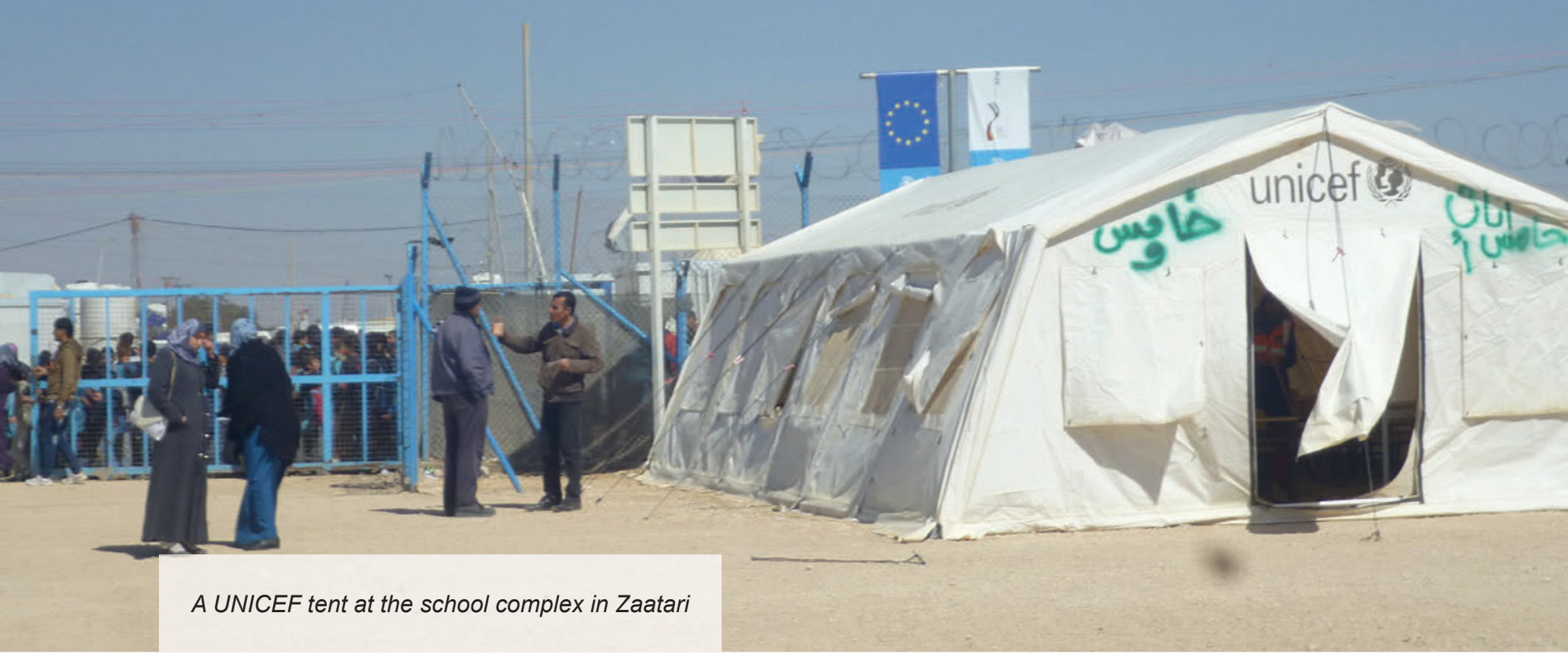

camps. UNICEF Education Officers provided planning, implementation, technical support, capacity building, and monitoring and evaluation. UNICEF is also working with its partners in refugee camps and in host communities to create and develop alternative education opportunities for children (further detailed below). NGO partners provide implementation and technical expertise. Donors, including the United States, the EU and others, provide funding.

From April 2012 until the early part of 2013, many anticipated that an end to the Syrian crisis and the return of Syrian refugees to their own country was imminent. However, as the numbers of refugees increased rapidly, and the likelihood of a quick solution diminished, longerterm educational needs became apparent. Of the registered refugees in Jordan, 36 per cent are school-age boys and girls (aged 5-17). ${ }^{8} \mathrm{~A}$ future stable and prosperous region depends on ensuring that these children are educated. Increasing access to schools in camps and host communities thus became a top priority. In March 2013 only 40 per cent of children eligible for formal education were enrolled ${ }^{9}$; attendance rates were probably lower. Low enrolment was linked to limited spaces in schools, psychosocial issues, ineligibility due to months of missed education, missing the start of the school year, transportation costs and factors related to early marriage for girls and seeking work for boys. ${ }^{10}$ These issues are discussed more fully Chapter 3 .

In response, the EER delivered a number of activities through a variety of organisations. By 
the 2014-2015 school year, enrolment in formal education reached nearly 60 per cent. ${ }^{11} \mathrm{~A}$ number of factors drove the improvements to access to formal education and other learning opportunities.

First, the Programme supported expansion of formal education. New school facilities were developed, with three new schools in camps and prefabricated classrooms erected in 28 schools outside the camps. Some 98 schools in host communities were double-shifted. During 2013 almost 2,000 recently appointed teachers working the afternoon shift in doubleshifted schools received training and $450 \mathrm{MOE}$ officials received capacity building training. ${ }^{12}$ The EER also provided school supplies. UNICEF orchestrated a successful campaign to encourage enrolment (implemented by Save the Children Jordan).

Second, the Programme provided alternative education (both non-formal and informal) to 35,000 vulnerable children in both camps and host communities. Non-formal education provides a certified formal education for older students through a drop-out programme which started in early 2015 , an adult literacy programme which started in early to mid2015 (both provided by UNICEF), and homeschooling, evening studies and summer study programmes (provided by NGOs). Nonformal education has been expanded through Questscope's 47 Centres (co-managed by MOE), and Islamic Relief Worldwide has an accelerated learning programme (ALP) which includes a contribution towards rent per child attending. Informal programmes include a variety of activities promoting life skills, numeracy and literacy; some programmes have a community focus as well as an educational one. In 2015, the
Makani initiative was established to reach 90,000 children excluded from education and exposed to risks. This is planned to be rolled out through 200 centres nationwide with assistance from a variety of NGOs. ${ }^{13}$

Third, the Programme addresses gender, special learning and disability needs through its programmes and builds on the capacity of the GOJ and NGOs to provide inclusive formal, informal and special education. The Programme also provides psychosocial and WASH support in both camp and host community education settings.

Fourth, efforts were made to improve quality and management. At the national level, these efforts are coordinated by the MOE and relevant government partners. The Education Sector Working Group (ESWG), chaired by UNICEF and Save the Children International and supported by the MOE, coordinated the activities of partners implementing the Programme. In early 2015 the ESWG adopted the INEE Minimum Standards for Education ${ }^{14}$ (although the impact of this is yet to be seen).

Finally, an Education Management Information System (EMIS) is being developed by UNESCO (following a MOE request) to support educational data needs.

Despite improving access, substantial problems have yet to be resolved. These are detailed more fully later but, in brief, 40 per cent of children remain without access to formal education; there is widespread overcrowding in both host community and camp schools - teacher/ student ratios of 1:60 are not unusual ${ }^{15}$; in the host communities, schools may be located too far from homes and journeys may be

11 UNICEF (2013d); UNICEF (2014a).

12 UNESCO (2013).

13 UNICEF (2015).

14 Education Sector Working Group (2015).

15 MOPIC (2014a). 
expensive or unsafe; bullying is widespread; teachers struggle with challenges they face in the classroom; and few are trained in dealing with the psychosocial problems of their pupils. In double-shifted schools, instructional time is shortened and amenities are poor. More recent efforts to increase school capacity have resulted in a diverging approach between two major organisations: the MOE prefers donors to fund the building of new schools in areas with large refugee populations, while UNICEF prefers an approach based on improving existing facilities and building prefabricated classrooms.

However, interviews and focus group discussions conducted for this evaluation show that Syrian children and their parents very often remain convinced of the importance of education and participate when the opportunity arises. The partners in delivering education for these children are progressively identifying and responding to not only issues of access but also issues of quality, and as the crisis becomes longer term, moving from a short-term response to an immediate crisis towards efforts to build resilience and sustainability. How successfully this is being managed is addressed in the following chapters.

\subsection{Evaluation purpose and objectives}

The purpose of this evaluation is to provide reliable evidence on the EER to support judgments about its current performance, give examples of good practice, and identify lessons to strengthen future improvements. The primary audiences are the GOJ (particularly the MOE), UNICEF and its implementing partners, other UN agencies, donors and the ESWG. The findings will be relevant to all those with an interest in assisting, sustaining and building the resilience of Syrian refugee children in Jordan through improved access to, quality and coordination of education provision. The findings will also be relevant to stakeholders in refugee crises in other countries.
This evaluation provides an analytical review of progress achieved in implementing the EER to date. It assesses performance against the criteria of relevance, effectiveness, efficiency and sustainability, and in line with UNICEF's Core Commitments for Children in Humanitarian Action. It examines cross-cutting issues and crosssectoral linkages including human rights-based approaches, gender equality, protection and psychosocial support, and WASH. The evaluation is underpinned by a focus on achieving improved outcomes for Syrian children in Jordan.

However, the yardstick by which to measure a programme of this sort is neither self-evident nor unchallengeable. If success were measured against the high standards humanitarian agencies set for themselves, then the answer would almost certainly be that the programme has failed, as evidenced by children who remain out of school, poor learning environments in schools, limited alternatives to formal school, child labour and early marriage. On the other hand, if the focus is on what was feasible and achievable during a massive crisis with limited resources available, then the answer is very different, as evidenced by the remarkable expansion of school places, creative alternatives to education, psychosocial support for many children, resources raised and so on. This evaluation remains agnostic between these approaches but will form judgments based on an understanding of the realities of delivering support in difficult circumstances as well as the relevance of a vision of a future in which children's rights are fully realised.

\subsection{Evaluation scope and focus}

This evaluation assesses the EER Programme's performance in providing access to formal education in camps and host communities, the development of alternative educational programmes, the creation of supportive programmes and systems, and the minimisation of impact on the Jordanian host community. It assesses these components of the education 

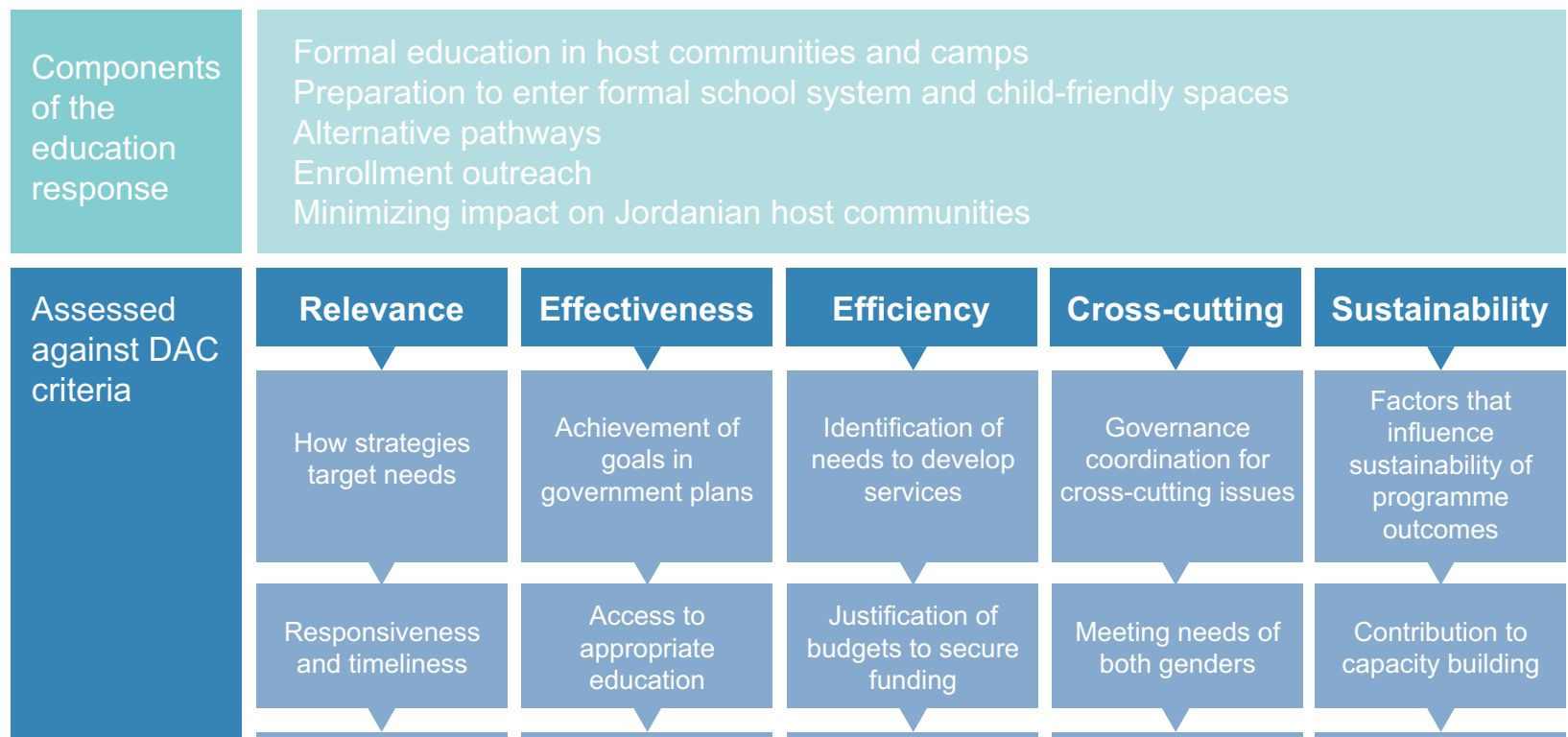

Contribution to

capacity building
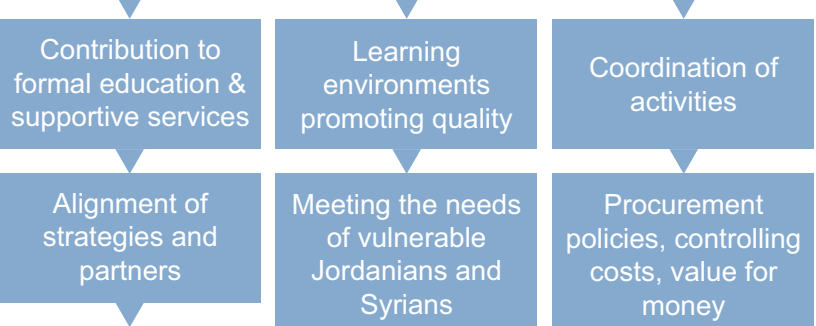

Promoting a

protective learning

environment

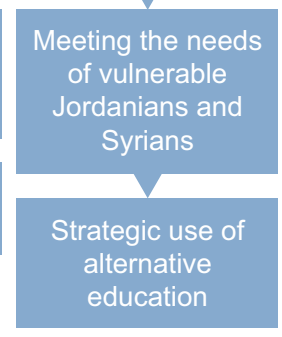

Procurement

policies, controlling

costs, value for

money

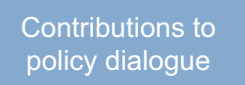

policy dialogue
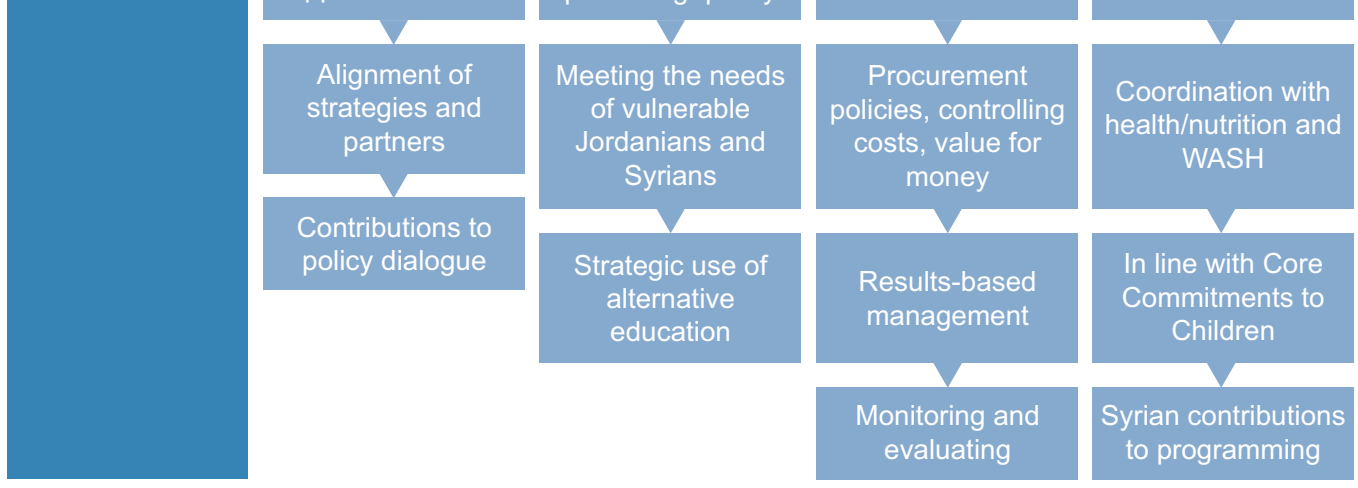

UNICEF's efforts to

ensure staff capability

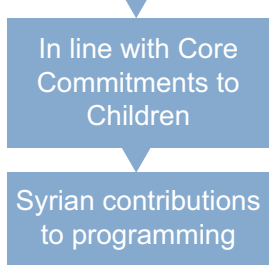

Monitoring and

evaluating

to programming
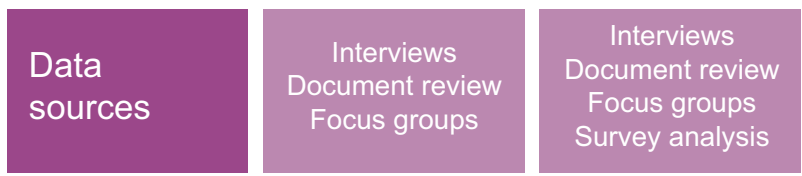

Interviews

Document review

Focus groups

Survey analysis

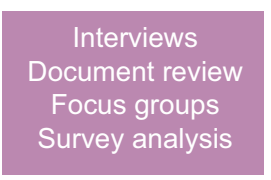

Interviews

Document review

Focus groups

Figure 1.1. Components of the education response, evaluation criteria and questions, and data sources

response against the commonly used DAC evaluation criteria ${ }^{16}$ of programme relevance/ appropriateness, effectiveness, efficiency and sustainability, while taking into account the evolving context. Within each of the DAC evaluation criteria, the evaluation addresses a number of specific points agreed with UNICEF, summarised in the blue boxes in Figure 1.1 and further elaborated in Chapters 2-6. Annex 1 presents a consolidated summary table of evaluation questions and findings. 


\subsection{Methodology and limitations}

This evaluation relied on both quantitative and qualitative methods. Figure 1.1 depicts how methodology contributed to evaluation questions; further detail on methodology and ethics is given in Annex 2.

First, the evaluation drew on a review of publically available literature, historical practice review, UNICEF internal documents and documents from partners.

Second, it was informed by 21 interviews with 39 education stakeholders from the GOJ, UN agencies, donors, local and international NGOs, community and religious groups operating in camp and urban settings, municipal education authorities, education camp managers, and principals of schools with Syrian refugees as students.

Third, 62 focus groups were conducted with children, parents and teachers. These were used to contribute to analysis of access to and quality of formal, non-formal and informal education, as well as supportive and cross-cutting services. Participatory photography (based on the principles of PhotoVoice ${ }^{17,18}$ ) followed by group dialogue in some of the focus groups allowed the evaluation team to deepen their understanding of the Syrian refugee children's experience pertaining to schooling and education. Photographs taken by Syrian refugee children, accompanied with the child's description as a caption, are interspersed within the report.
Fourth, the evaluation includes RAND's secondary analysis of Zaatari and host community Joint Education Needs Assessment (JENA) survey data. For this, the evaluation statisticians conducted basic, largely descriptive statistical analysis to portray the education landscape in Zaatari and host communities. The analysis was limited by the original data, ${ }^{19}$ but nevertheless provides useful contextual evidence. In many cases, results confirmed findings from other studies and from elsewhere in this evaluation. By triangulating evidence from multiple sources, a rich picture of refugee education has emerged.

The evaluation's data collection and use was supervised with formal ethical review by RAND's Human Subjects Protection Committee (HSPC). The evaluation is also in line with UN principles for integrating human rights and gender equality in evaluation.

This evaluation has several limitations. First, it focuses on registered refugees; due to the challenges of accessing and identifying unregistered refugees, this group is not included in the evaluation. Second, it allows judgments to be formed about the performance of the EER as a whole, but it does not evaluate it in detail in each of Jordan's 12 governorates, as data were not designed or available to analyse how the situation varies among locations in Jordan. This evaluation drew on data from on two refugee camps (Zaatari and Azraq) and the five governorates of Irbid, Mafraq, Amman, Karak and Zarqa.

17 PhotoVoice (2015)

18 The evaluation team is aware of the UNHCR TRACKS 'Picture This' project which equips, trains and encourages refugee children in camps to document their daily lives through photography and keeping photo journals and would like to thank the workshop leader Brendan Bannon for his advice. See UNHCR TRACKS (2015).

19 The Zaatari Camp data included a relatively small sample size, with a consequently smaller sample size for particular groups. However, the sample size was sufficiently large to allow multivariable analysis as a predictor of attendance in formal education. Zaatari Camp Needs Assessment used random sampling of households with children aged 3-18 based on GPS coordinates (sample size: 1,125 children). In the host community data, data collectors took a non-probability approach to the sampling of Syrian households, based on a sampling frame from the World Food Programme, a keyinformant network, and snowball sampling of households within communities (sample size: 9,085 children from 3,327 households). This is not an appropriate approach for formal statistical inference; however it was a pragmatic and practical approach to identifying the relevant population given the context. 


\subsection{Organisation of the report}

This report is organised into seven chapters. In line with the DAC evaluation criteria, Chapters 2-6 discuss findings and draw broad conclusions, integrating the document and literature review, stakeholder interviews, focus groups and survey data analysis. Chapter 2 discusses findings about relevance, calling for a shift to a longer-term, evidence-based strategy. Chapter 3 provides analysis about effectiveness, concluding that there is a crisis of access for out-of-school children, that there are ways to improve quality, and that alternative programmes should be better targeted. Chapter 4 discusses efficiency, making suggestions for prioritising budget categories, improving funding transparency and gathering data to better compare value for money. Chapter 5 examines how cross-cutting issues interact with education, suggesting additional approaches to gender, psychosocial provision and disability support. Chapter 6 delineates factors enabling sustainability, proposing a sustainable 'platform' of supporting systems and describing trade-offs. Finally, Chapter 7 concludes with recommendations based on findings from the previous chapters. 



\section{Findings: relevance}

The DAC criteria $^{20}$ define relevance in development assistance as: 'The extent to which the aid activity is suited to the priorities and policies of the target group, recipient and donor'; they include consideration of whether objectives are valid and whether activities support those objectives. To this end, the specific evaluation questions discussed in this chapter (shown in Table 2.1) focus on the strategy of the Emergency Education Response. The chapter starts by discussing availability of strategy and Theory of Change (ToC) documents. Then it describes factors that influenced and constrained the development of a strategy that targets relevant needs. It concludes that EER efforts resulted in a significant and rapid provision of structured education; the initial response was highly appropriate However, with the transition towards providing education for a long-term crisis, strategic efforts and planning lag behind events on the ground. Lack of a clear, prioritised and coordinated strategy going forward will not enable the Programme to meet objectives.

Table 2.1 below provides a quick-reference overview of how the Programme has met its objectives in relation to relevance, structured around the evaluation questions posed by this report. These ideas are explored in more detail in the sections that follow.

Table 2.1: Relevance - overview of evaluation questions and findings

\section{Evaluation questions}

- How strategies target needs

- Relevance and appropriateness in responding to needs of displaced Syrians and vulnerable Jordanians

- Responses to unforeseen contingencies, and timeliness when coordinating with other agencies

- Strategy contribution to formal education and supporting services

- Contribution to policy dialogue

- How strategies target needs of service providers

- $\quad$ Alignment of Jordanian, UN and wider regional strategies

- Alignment of donors and partners

\section{Overview of findings}

Activities in 2012-2013 were highly relevant, creating a rapid response to educational needs enabling many displaced Syrian children to enrol in school with supporting services. The scope and protracted nature of the crisis were not foreseen. As time passed, strategic development did not keep pace with events.

While there are high-level documents (RRP6 and 3RP) that describe objectives, needs and activities, there is no single document that explicitly lays out a strategy or Theory of Change for the education response, formal education and supporting services.

The GOJ, donors, UNICEF and service providers developed structures to discuss policies, as well as overarching strategy documents of RRP6 and 3RP. Strategy development, contribution to policy dialogue, alignment with wider regional and national strategies, and alignment of donors and partners (GOJ, UN agencies and services providers) were influenced and constrained by: complex, interacting components; rapidly changing circumstances; lack of consensus on time frame and approach to what is relevant in the longer term; being relevant to a largely urban crisis in a middle-income country; and tensions between a nationality-based approach and a vulnerability or rights-based approach. These factors posed challenges to adapting the approach and activities in the face of changing circumstances and needs. 


\subsection{The EER lacks an explicit theory of change, limiting adaptation to a changing strategic context}

The EER evolved during its first 18 months from meeting urgent needs to focusing on resilience and sustainability. A series of planning documents lay out overarching strategies and approaches to the overall, multi-sectoral refugee response. The Regional Response Plan 6 (RRP6), drafted in 2013, presents a programme for 2014. The Regional Refugee \& Resilience Plan (3RP), drafted in 2014, presented a plan for 2015. The National Resilience Plan (NRP) 2014-2016 and Jordan Response Plan (JRP) of 2015 also present multi-sectoral plans (with the JRP superseding the NRP in 2015). ${ }^{21,22}$

For education, these strategic documents describe broad objectives, needs, a series of programmes, and budgetary requirements. In 2013, as described in Section 1.2, the continued scale and likely duration of the situation in Syria became clearer, The initial phase of opening borders and schools to Syrian refugees was followed by a strategy of scaling up to strengthen access, quality and protection. In response, the RRP6 described two primary objectives for emergency education in Jordan:

1. 'Children and youth have sustained access to appropriate education opportunities.'

2. 'Children and youth benefit from learning environments that promote quality education, protection and their well-being. ${ }^{23}$

The overarching education objective of the 3RP is similar, describing goals of access, quality and protection, while adding emphasis to the needs of vulnerable Jordanians as well: 'to ensure sustained quality educational services for all refugees and for vulnerable Jordanians affected by the crisis. ${ }^{24}$ The $3 R P$ describes the greatest education sector problems as overcrowding of schools in high population density areas, shortages of qualified teachers, concerns about declining quality, violence amongst schoolchildren, and expansion of alternative education opportunities. The NRP signals a greater effort 'to complement and underpin humanitarian efforts by promoting a longer-term, development oriented approach' (p.12). The JRP reinforces this strategic shift, stating: 'The Jordan Response Plan (JRP) seeks to bridge the divide between resilience and humanitarian systems, and reconcile the programming objectives, funding mechanisms and operating systems that often run parallel to each other in addressing short-term people-centred needs, in addition to medium and longer-term systemic and institutional considerations' (p.6).

Furthermore, the Terms of Reference for this evaluation highlight three areas of emphasis for the EER:

1. 'Vulnerable school aged children access formal education opportunities and receive psychosocial support at schools in urban and camp settings;

2. Children excluded from formal education opportunities access alternative learning activities at the community level and in camp settings;

3. Effective monitoring and evaluation system established in collaboration with the MOE to monitor progress towards Educational and learning activities.'

\footnotetext{
21 See Regional Refugee \& Resilience Plan (2015) and Hashemite Kingdom of Jordan (2014). The NRP 2014-2016 can be accessed here: http://www.un.org.jo/sites/default/files/NRP.pdf

22 OECD (n.d.).

23 UN. (2013).

24 Hashemite Kingdom of Jordan (2014).
} 
In the early stages of the crisis, the relevance of the EER depended upon whether it met the initial perceived need to provide access and a place of safety. By 2014-2015, however, the relevance of the EER depended on wider agenda including access, quality, protection (including psychosocial support), alternative learning approaches, meeting needs of vulnerable Jordanians and 'medium to longer-term systemic and institutional considerations' that include monitoring and evaluation.

The EER is an example of a complex intervention. ${ }^{25}$ Good practice in both designing and evaluating complex programmes includes development of a theoretical understanding of the strategy (a Theory of Change ( $\mathrm{ToC}$ )) and of how the intervention causes change, so that weak links in the causal chain can be identified and strengthened. ${ }^{26}$ The benefits of developing a shared strategy and $\mathrm{ToC}$ across collaborating organisations include creating greater clarity and providing a tool to demonstrate to funders and other stakeholders the measurable value of the programme.

However, an explicitly articulated ToC or strategy for the EER is absent from its various planning documents. There are three dimensions to this. First, to provide coordination and alignment across the sector, the implications of the strategic shift towards resilience and longer-term institutional considerations needs to be explicitly taken into account in planning documents. Second, review of documents showed their content to be at a high level, without the detail needed to align activities and to connect the evolving high-level strategic goals with the specific education activities in camps and host communities. Stakeholders echoed this sentiment during interviews for this evaluation. For example, an NGO official noted in an interview that there had never been co- development of a strategy, although the 3RP process has committees planning to address some of these gaps in the future. A donor interviewee called for a strategy to 'look at how we can link up education and emergency and the formal systems in Jordan and the region.' A UN official called for a roadmap to align the plans of all partners, so that education actors could coordinate their activities. Third, documents lack prioritised budgets. While some interviewed for this evaluation thought that the 3RP was groundbreaking in creating a governmentled, unified and overarching strategy for the multi-sectoral refugee response, some donors criticised it as 'nonsense' and a 'wish list and not a strategy', with too much emphasis on conducting activities favoured by the service providers as opposed to focusing on evidencebased needs.

Despite this, it is important to recognise that, as described in Section 1.2, the EER partners did achieve a shift of focus onto improved training, addressing issues of bullying and violence, identifying infra-structural issues, and exploring longer-term solutions to the problems faced by children in and out of formal education. Training of teachers and the development of minimum standards (also described in Section 1.2) are further indications of how the EER has remained relevant to a changing national strategic focus. However, efforts to remain relevant within a changing strategic context have faced challenges, and these are outlined in the following section.

\subsection{There are significant challenges to the EER being and remaining relevant}

According to the document review and stakeholder interviews, several factors, discussed below, created challenges in

25 Ling (2012).

26 Moore et al. (2014). 
developing and maintaining a relevant strategy. These factors influenced and constrained policy dialogue, alignment with strategy documents, alignment of donors and partners, and meeting the needs of service providers.

\section{Complex, interacting components}

Programme relevance depends upon aligning interacting components, including: advocacy, fund-raising, identification of needs, design of appropriate and prioritised responses to these needs, and creation of funding mechanisms to ensure responsiveness, quality programming and sustainability. Multiple organisations contribute, including the GOJ, UNICEF and other UN agencies, donors, and UNICEF implementing partners. The GOJ, the ESWG and UNICEF all have a coordinating role in keeping this diverse set of agencies aligned within a single, relevant programme.

Relevance of decisions made is likewise challenged by disparate and at times conflicting agendas, values and goals among donors, UN agencies, the government and implementing partners. While all stakeholders want to do the best for the education of children, there are significant unwritten differences in priorities and values, and interviews indicate that communication between key stakeholders at this level is often lacking. Among some UN interviewees, there was a sense that differing government and UN priorities led to inconsistent requests to donors and disparate donor agendas.

\section{Rapidly changing circumstances}

As described in the previous section, the environment in Jordan has changed significantly, and continues to evolve. The circumstances of the creation of the Programme called for a fast response and did not allow for the development of an explicit ToC that kept pace with the changes on the ground and unforeseen contingencies. With a large number of actual and potential partners, with challenges facing effective parental and community participation, and with donors confronting increasing demands on their resources, remaining relevant through a flexible and aligned response has proved difficult (as discussed in more detail in the Chapter 4).

\section{Lack of consensus on time frame and approach to what Is relevant in the longer term}

What might be relevant as a short-term response might be less relevant over a 5- or 10-year period. According to MSF, 'The scale of the crisis was initially under-recognised, mistakes were made in the planning stages... and also the relief effort lagged behind the numbers and needs.... ${ }^{27}$ This has undercut efforts to achieve agreement on the infrastructural investments needed, whether investment in new schools or the rehabilitation of schools with double-shifting. Addressing the longer-term specific material, legal, educational and psychological needs of Syrian children and affected Jordanian children entails 'making decisions focused on the long-term operational relevance of emergency interventions', as concluded by a report on Syrian refugee education published by Oxford University. ${ }^{28}$

A UNICEF planning meeting document concluded: 'Given the scope and scale of the humanitarian response for Syrian refugees, there is a growing sense that it is not possible to continue the response as is. A greater shift to system strengthening is required, making it possible for the existing systems and institutions 


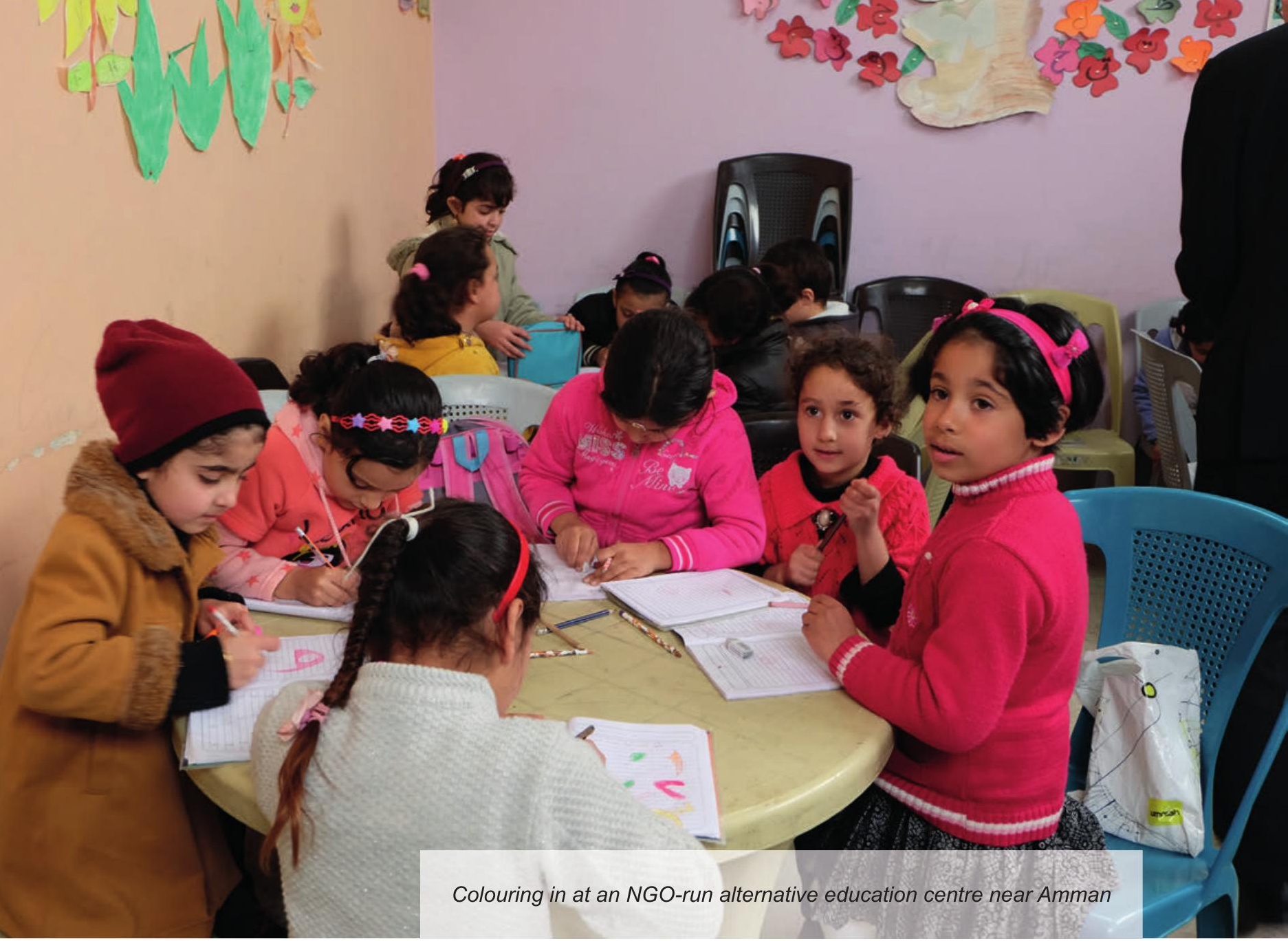

in the country to provide services to Jordanians, Syrians and other children alike. Accordingly partnership should move from a project-based to a more system-wide, holistic approach. ${ }^{29}$ The emergency response invested in short-term partner programmes that provided much needed capability and a fast response; however, a 'more system wide, holistic approach' with longer-term goals has yet to emerge. This will become more urgent should donor funding diminish.

It is in this context that many UN, NGO and government stakeholders interviewed who recognised the need for such a strategic shift noted that it is not underway. As one government stakeholder stated: 'We need to work together with the donors, UNICEF and the government, to speak the same language... to speak the needs of Jordan.' Despite the dialogue around a shift from emergency to resilience, interviews indicate that there has been no clear approach to transition. Short donor cycles limit the ability to invest in longer-term programmes, affecting efficiency, and were raised across interviews as a major barrier to sustainable planning and programming. Formal education is missing a long-term explicit commitment from donors and an acceptance of shared burden for longterm improvements. Implementing partners are incentivised to conduct visible projects of their own as opposed to building local capacity. As one NGO partner commented, 'Longer term planning would allow for more sustainable, long-term impact and better quality... it should be part of our goal to change education policy and training, but we're still in the emergency mode. It needs to change to a development response.' What is meant by a system-wide, holistic approach in this context is discussed in Chapter 6.

\section{Being relevant to a largely urban crisis in a middle-income country}

In many historical situations of refugee crises (for example, in Somalia or Afghanistan), already poor communities in failing states were displaced, seeking refuge in other weak states. Jordan is a middle-income country with 
a well-functioning state, and before the war Syria was a middle-income country. In Jordan, the vast majority of the registered Syrian refugee population (84 per cent) lives in host communities, outside of the refugee camps that have been established. ${ }^{30}$ This means a different kind of response is needed for Jordan than in other situations and that the bulk of the response is needed for urban areas, in addition to camps. UNHCR issued guidance on adapting emergency responses to urban areas in 2009, ${ }^{31}$ and several years later found challenges transitioning the international skill base, developed in camps, to urban areas. ${ }^{32}$

As an NGO partner noted in interview, the response community was used to working in refugee situations in Africa and other parts of Asia and were bringing those skills to Jordan, with both advantages and disadvantages. Another NGO official said, 'The humanitarian sector has come with a package that was African.... It is getting better, but these NGOs have packages which are based on research based elsewhere.' Another elaborated, 'Implementing partners need further understanding of community needs to provide relevant services.' The advantages included the international community knowing how to operate camps and NGOs knowing how to offer parallel services. The international community is less familiar with supporting the existing capabilities of national organisations in a functioning state with a robust, albeit imperfect education system. Specifically, this has resulted in tensions over infrastructural spending, described above, and a concern that Jordanian NGOs and civil society organisations have been bypassed in favour of international agencies (outlined in Chapter 6).

\section{Tensions between a nationality-based approach and a vulnerability or rights- based approach}

Understandably, the refugee education response focuses on Syrians. However, government and NGO interviewees noted that there are vulnerable Jordanians as well who do not get the same attention. Tensions over how assistance is allocated are exacerbated by the fact that the Syrians and Jordanians often live integrated together in urban settings. The GOJ has mandated that a share of programmes for the Syrians also target vulnerable Jordanians. According to a wide range of interviewees, there still remain tensions between a 'vulnerabilitybased' and a 'nationality-based' approach to programming. As the conflict in Syria continues, this emphasis will become increasingly important to the sustainability and stability of educational options and of Jordanian society as a whole. UNICEF's for Children (CCC) in Humanitarian Action $^{33}$ frame its efforts to protect the rights of children in all its activities, more specifically those enshrined in the Convention on the Rights of the Child. ${ }^{34}$

\subsection{Developing a theory of change}

According to good practice in the evaluation literature, the evaluability of complex programmes $^{35}$ in the absence of a clearly articulated ToC can be compromised. Outlining

30 As of September 2014, there are 521,500 refugees residing outside of camps. As stated in the Jordan Response Plan Refugee Pillar Needs Assessment 061014 (provided to evaluation team).

$31 \quad$ UN Refugee Agency (2009)

32 Morand et al. (n.d.)

33 UNICEF (2010a).

34 Office of the High Commissioner for Human Rights (1989).

35 Davies (2013). 
a ToC for the Emergency Education Response therefore involves making what is implicit explicit and is a necessary framing device for this evaluation. However, it is less satisfactory than using an existing and agreed $\mathrm{ToC}$ developed by the stakeholders involved.

To develop an explicit Theory of Change for the Emergency Education Response, the evaluation draws from several key documents: the Regional Response Plan 6 (RRP6), the Regional Refugee \& Resilience Plan (3RP), ${ }^{36}$ the National Resilience Plan (NRP) 2014-2016 and the Jordan Response Plan (JRP) 2015, ${ }^{37,38}$ UNICEF internal planning documents, and the Paris DAC Declaration. ${ }^{39}$ Figure 2.1 lays out the proposed Emergency Education Response Theory of Change based on analysis of these documents, including a description of the vision of the refugee response, the vision of the Programme, medium-term and immediate change goals, strategies, drivers and barriers, inputs and principles. The $\mathrm{ToC}$ also includes considerations for longer-term systems strengthening.

\subsection{Summary and conclusions}

The DAC criteria for relevance include whether objectives remain relevant over time and how well activities target those objectives. Considering these criteria as well as the specifics of this evaluation (shown in Table 2.1), this evaluation describes how the EER Programme was relevant to the needs of a large number of Syrians arriving unexpectedly in Jordan. Once the scale and likely longevity of the situation became apparent, activities were scaled up. As the strategic context evolved the organisations in the EER recognised the need for a re-orientation towards resilience and development but, faced with significant barriers, progress towards this was incomplete. Given these challenges, there are particular ways in which the Programme's strategy and objectives need to be adjusted for relevance to meet changed circumstances:

- Clarifying longer-term roles and responsibilities.

- Recognising the medium- to long-term nature of refugee education needs in Jordan.

- Moving from short-term emergency responses to longer-term resilience and development planning, including strengthening systems in Jordan in addition to immediate service provision.

- Creation of a shared set of strategic expectations and trade-offs among stakeholders.

- Prioritisation of activities based on availability of resources.

- Applying skills, resources and approaches in a way that recognises the predominantly urban nature of the crisis, in addition to camps.

- Balancing approaches to target vulnerability instead of nationality, acknowledging that most of the vulnerability is among the Syrians while there is also vulnerability among the Jordanians.

No single strategy document lays this out for the education sector currently. Such a document would strengthen the relevance of the programme, support the alignment of different agencies and better enable the work of service providers. It would provide, at this moment of transition, an opportunity to step back and take stock. The following chapters discuss these issues in more detail.

\footnotetext{
36 See Regional Refugee \& Resilience Plan (2015) and Hashemite Kingdom of Jordan (2014).

37 The NRP 2014-2016 can be accessed here: http://www.un.org.jo/sites/default/files/NRP.pdf and the JRP here: http://www.undp.org/content/dam/rbas/doc/SyriaResponse/3\%20RP\%20Jordan.pdf

38 OECD (n.d.).

39 OECD (n.d.)
} 
Vision of Syrian

programme

Vision of EER
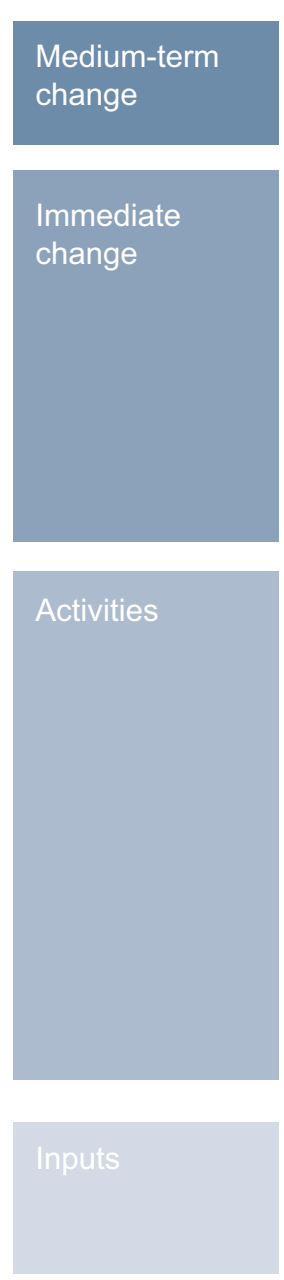

All those affected by the Syrian crisis in Jordan are protected and empowered

All affected children and youth have sustained access to appropriate education opportunities Children and youth benefit from learning environments that promote quality education, protection and their well-being

A coordinated, efficient and prioritised multi-agency education response delivers inclusive and participatory services, efficiently and sustainably meeting humanitarian standards across all sectors, targeted on greatest need and equitable across all sectors

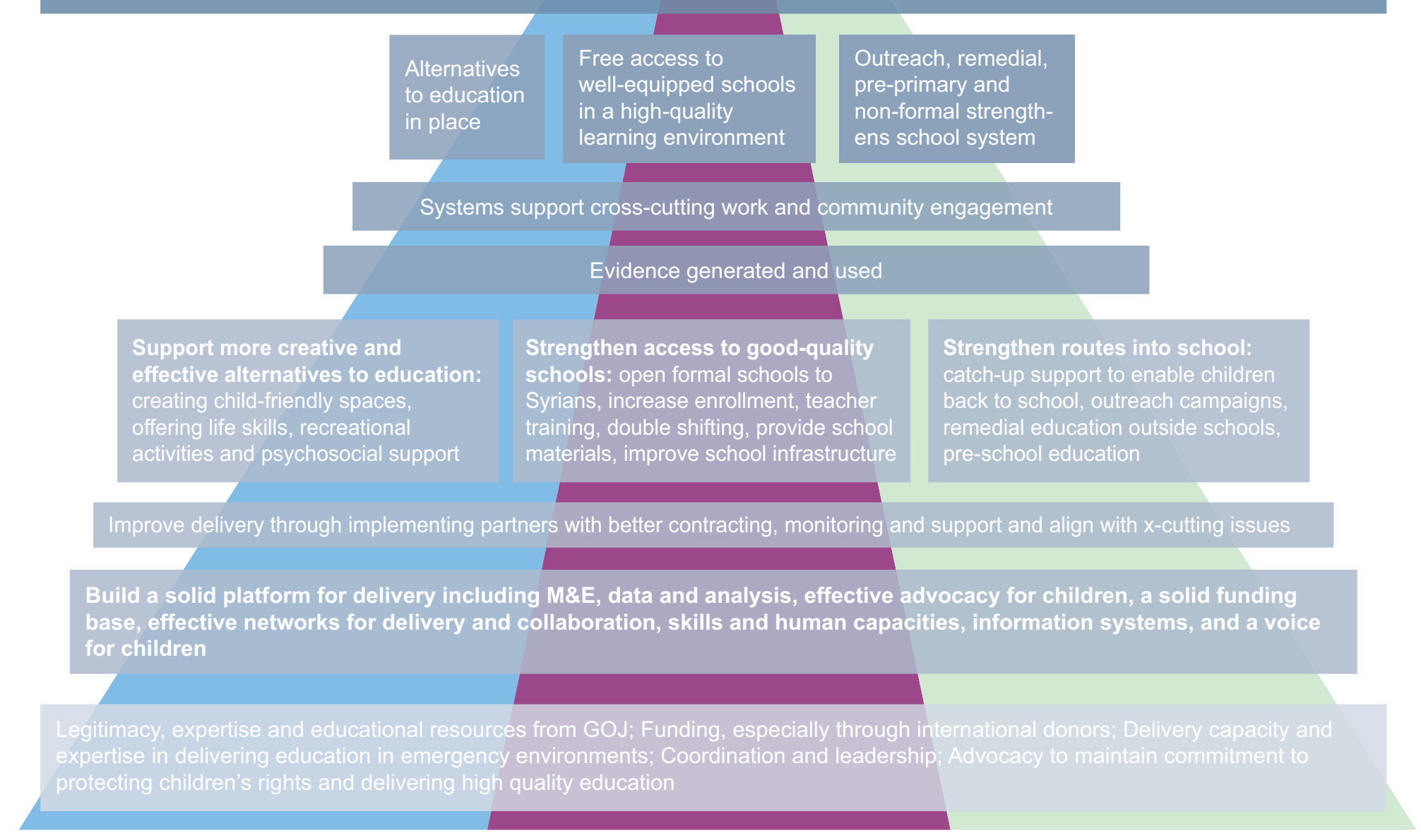

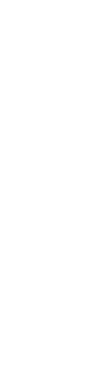

Figure 2.1: The proposed Emergency Education Response ToC for this evaluation 


\section{Findings: effectiveness}

The DAC criteria define effectiveness as 'a measure of the extent to which an aid activity attains its objectives. ${ }^{40}$ In line with this, several specific evaluation questions (Table 3.1) frame this assessment of the Programme's effectiveness, including how well the programme met the objectives established in relevant government plans (the RRP6, 3RP, JRP and NRP): providing sustained access to appropriate education, creating learning environments promoting quality and protection, alternative education approaches, and meeting needs of vulnerable Jordanians as well as Syrians. Given the shift to 'resilience' in recent plans, consideration of effectiveness is also framed by how the Programme addresses 'the divide between resilience and humanitarian systems, and reconcile(s) the programming objectives, funding mechanisms and operating systems that often run parallel to each other in addressing short-term people-centred needs, in addition to medium and longer-term systemic and institutional considerations' (JRP, p.6).

This chapter begins with a discussion of access to formal education, including the challenges of meeting the needs of out-of-school children. It then discusses quality of formal education, and the effects of the additional children on the Jordanian host community. Next it describes alternative education approaches (non-formal and informal) that have aimed to address educational needs not met through the formal schools. It concludes that significant achievements have been made in providing access to formal education, but that there remain shortcomings in both access and quality of formal education for the Syrian refugees. Some alternative education programmes have demonstrated high quality, but need more strategic focus. Current approaches to meeting the needs of the refugees are detrimental to meeting the long-term educational needs of vulnerable Jordanians. These findings suggest changes to improve the effectiveness of programming. (Chapter 5 focuses on effectiveness in other aspects of education, with an emphasis on protection, psychosocial support and rights.)

Table 3.1 overleaf provides a quick-reference overview of how the Programme has met its objectives in relation to effectiveness, structured around the relevant evaluation questions posed by this evaluation. These ideas are explored in more detail in the sections that follow.

\subsection{Access to formal education}

The GOJ, UNICEF and implementing partners have taken a number of steps to provide access to education. This section describes those steps and resulting access rates. It then analyses access among different sub-groups of children, by location, by characteristics of educational provision, and by characteristics of the child, relying on triangulation of findings from the survey data analysis, document review, stakeholder interviews and focus groups with children, parents and teachers. Next it describes effects on out-of-school children. Finally it concludes that despite efforts to promote access, there remain significant gaps in this area. 
Table 3.1: Effectiveness - overview of evaluation questions and findings

\begin{tabular}{|c|c|}
\hline Evaluation questions & Overview of findings \\
\hline $\begin{array}{l}\text { Achievement of goals in } \\
\text { RRP6, 3RP and relevant } \\
\text { government plans }\end{array}$ & $\begin{array}{l}\text { Objectives stated in RRP } 6 \text { and } 3 R P \text { included access to education, quality and } \\
\text { protection of education and meeting the needs of vulnerable Jordanians. Findings for } \\
\text { each are summarised in the lines below. }\end{array}$ \\
\hline $\begin{array}{l}\text { Equitable access to } \\
\text { appropriate education }\end{array}$ & $\begin{array}{l}\text { Providing access to formal education for } 130,000 \text { Syrian children is a significant } \\
\text { accomplishment of the education response. However, having at least } 60,000-97,000 \\
\text { Syrian children out of school requires urgent action. Improving access depends } \\
\text { on understanding determinants of access that include location, characteristics of } \\
\text { education provision and characteristics of the family and child. }\end{array}$ \\
\hline $\begin{array}{l}\text { Learning environments } \\
\text { promoting quality }\end{array}$ & $\begin{array}{l}\text { Quality has been a secondary consideration to access. While Jordan has a robust } \\
\text { school system, quality of education provided to the Syrian refugees has been below } \\
\text { desired levels. Many of the quality challenges have affected Jordanians as well as } \\
\text { refugees. While there are few ways of measuring quality, it is known that: test scores } \\
\text { are low; classrooms are crowded; teachers struggle in difficult environments; facilities } \\
\text { are in need of maintenance; Syrian students struggle in making the transition from } \\
\text { Syrian to Jordanian curricula; there are inconsistent policies about grade placement; } \\
\text { and unclear pathways to adulthood demotivate students. On the one hand, double- } \\
\text { shifting has provided opportunities to meet the quality needs of the Syrians and } \\
\text { Jordanians, but on the other double shifts in Jordan work worse than elsewhere. }\end{array}$ \\
\hline $\begin{array}{l}\text { Meeting the needs of } \\
\text { vulnerable Jordanians and } \\
\text { Syrians }\end{array}$ & $\begin{array}{l}\text { Additional influxes of students into Jordan's formal system have crowded out } \\
\text { resources for quality improvements for Jordanians, and classroom conditions have } \\
\text { become more crowded. Vulnerable Jordanians feel that they receive less attention } \\
\text { than vulnerable Syrians. }\end{array}$ \\
\hline $\begin{array}{l}\text { Strategic use of alternative } \\
\text { education }\end{array}$ & $\begin{array}{l}\text { Alternative education has been provided for } 35,000 \text { Syrian refugee children, and } \\
\text { providing such access has been more effective in camps than in host communities. } \\
\text { Alternative provision is perceived to provide high-quality, relevant, child-centred and } \\
\text { flexible education to Syrians. However, there is a lack of a clear framework for what } \\
\text { alternative education is intended to achieve over the longer term, as well as a lack of } \\
\text { consistent monitoring of quality, meaning that standards and achievements are hard to } \\
\text { measure. }\end{array}$ \\
\hline
\end{tabular}

Meeting learning needs There are no holistic learning needs assessments.

assessments

\subsubsection{An overview of access programmes and resulting access rates}

There were a number of approaches taken to achieve access, allowing the proportion of enrolled refugee children and youth to rise quickly from the start of the crisis in 2012 to the winter of 2013-2014, as noted in Section 1.2. First, the GOJ opened up the Jordanian public school system to Syrian refugees in host communities, and UNICEF and the GOJ collaborated in opening up schools in refugee camps. Outside camps, absorption of the Syrian students into the formal education system has occurred through adding Syrian students in Jordanian formal classrooms. In areas with many refugees, the MOE opened up Syrian-only second shifts in 98 schools. School buildings were also repaired and additional space rented; UNICEF reports that 69 schools were provided with furniture and equipment to support formal education and 65 schools with prefab classrooms. ${ }^{41}$ Other efforts also 
Table 3.2: Syrian refugee children attending types of education and out of school in 2014-2015

\begin{tabular}{|c|c|c|c|c|}
\hline $\begin{array}{l}\text { Integrated into } \\
\text { formal classrooms }\end{array}$ & $\begin{array}{l}\text { Enrolled in Syrian- } \\
\text { only second shifts }\end{array}$ & $\begin{array}{l}\text { Enrolled in formal } \\
\text { schools in camps }\end{array}$ & $\begin{array}{l}\text { Enrolled in } \\
\text { aternative education }\end{array}$ & Out of school \\
\hline 60,066 & 46,049 & 23,227 & Approx.35,000 & $\begin{array}{l}\text { At least } 97,000 \text { not } \\
\text { attending formal } \\
\text { education }\end{array}$ \\
\hline \multicolumn{4}{|c|}{$\begin{array}{l}\text { Total number of Syrian school-age refugee children in Jordan: } \\
\text { at least } 226,138 \text { ( } 36 \text { per cent of total population of Syrian refugees in Jordan) }\end{array}$} & $\begin{array}{l}\text { At least } 60,000 \text { not } \\
\text { attending any education } \\
\text { programme }{ }^{42}\end{array}$ \\
\hline
\end{tabular}

supported enrolment. Outreach and retention campaigns were employed widely in both camps and urban areas. The UNICEF-sponsored, Save the Children-run helpdesks in host communities and 'Back to School' outreach campaigns were highly visible and effective in encouraging enrolment. ${ }^{43}$ Catch up programmes and remedial programmes for children who had missed school were provided by extensive international efforts.

Based on the described measures, a significant number of children were provided with access to education, in a short time frame, with limited resources, and under conditions of significant uncertainty. At the same time, there still remain a large number of children out of school.

There are an estimated 226,138 school-age Syrian children in Jordan, based on the numbers of registered refugees. During 2014-2015, 129,354 Syrian children were enrolled in public schools (including those in camps), according to data provided to the evaluation team by the MOE. ${ }^{44} 60,066$ refugee children were integrated into single-shift host community schools; 46,049 were in Syrian second shifts, and 23,227 were enrolled in schools within camps. There are approximately 35,000 children enrolled in alternative programmes, designed as a supplement to formal education, not a replacement. Therefore, by these estimates, at least 97,000 school-age children are not attending formal school, and at least 60,000 are not attending any form of educational programming. The numbers of out-of-school children would no doubt be higher if unregistered refugees were considered. Furthermore, while no aggregate data is kept on attendance, interviews and focus groups with a wide range of stakeholders, including parents, children, NGO partners and school staff, indicate that actual attendance is lower than enrolment figures. Table 3.2 above shows these numbers.

\subsubsection{Access is correlated with a complex set of factors}

Despite achievements in creating access, there are still large numbers of out-of-school children. Analysis of the JENA data from host communities and Zaatari Camp, findings from interviews with stakeholders, and the results of the focus groups with parents, teachers and children reveals multiple determinants to access. These determinants can be divided into three categories: location, factors associated with provision of education, and factors related to circumstances of the family and child. Table 3.3 overleaf summarises these factors, and further discussion follows.

\footnotetext{
42 Estimates vary between the $\operatorname{NRP}(60,000)$ and the MOE $(95,000)$.

43 Save the Children Jordan (2014).

44 Data provided by UNICEF, July 2015.
} 
Table 3.3: Factors associated with children's access to education

\begin{tabular}{|c|c|}
\hline Location & 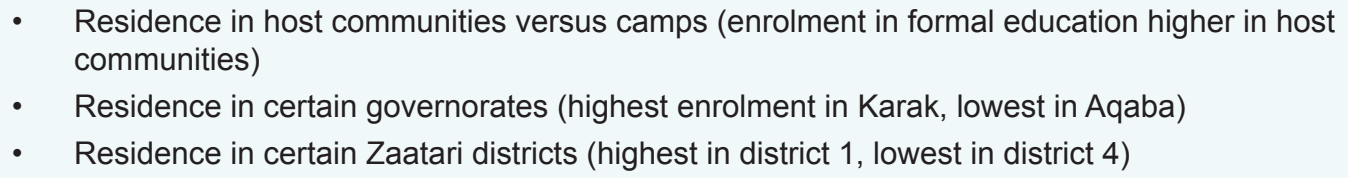 \\
\hline $\begin{array}{l}\text { Education } \\
\text { provision }\end{array}$ & $\begin{array}{l}\text { - } \text { Restrictive enrolment policies, based on years of education missed and arrival date } \\
\text { - } \\
\text { - } \\
\text { - }\end{array}$ \\
\hline $\begin{array}{l}\text { Household or } \\
\text { child }\end{array}$ & 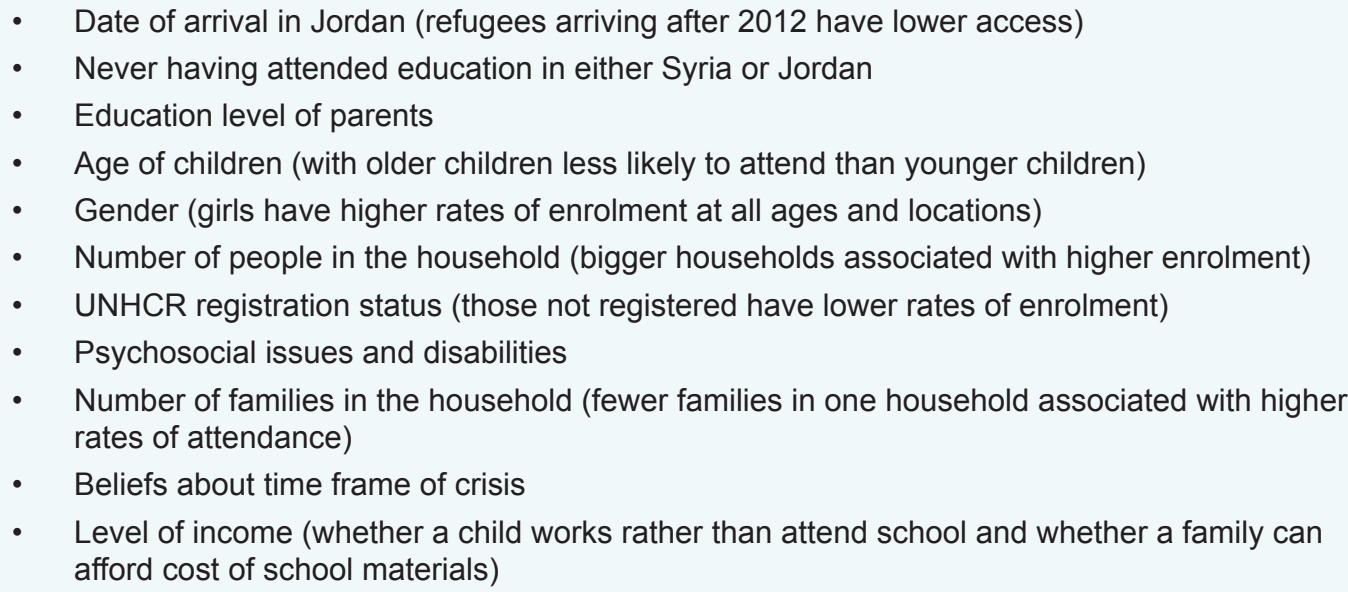 \\
\hline
\end{tabular}

\subsubsection{How access is associated with location}

\section{Several factors related to a Syrian refugee} child's location affect access to education. ${ }^{45}$ First, according to the secondary data analysis, attendance rates to formal education are higher in host communities than Zaatari. As Figure 3.1 illustrates, attendance in formal education among Syrian refugee children aged $6-17$ is 64 per cent in host communities and 52 per cent in Zaatari. Furthermore, there is a higher percentage of children in Zaatari who have never attended formal education (36 per cent) than in the host communities (11 per cent).
JENA data shows drop-out rates from formal education for Syrian refugee children aged 6-17 to be higher in Zaatari Camp (at 6 per cent) than they are in the host communities (2 per cent of children without disabilities, in the last 6 months); however, this conflicts with analysis by Chatty et $\mathrm{al}^{46}$ (2014, pp. 30-31) that shows dropout rates to be 20 per cent in the camps and 27 per cent in host communities.

Higher attendance in the host communities than in Zaatari is at first sight surprising, as intuitively it may seem easier for education partners to reach refugees in camps than in host communities. There may be several reasons for

\footnotetext{
45 There are limitations to the data about access. The JENA survey questions asked about 'attendance', while describing it in a way that implied enrolment; no actual data about children's day-to-day attendance is available. The discussion below uses 'enrolment' or 'attendance' as it was used in the survey data, with the caveat that this distinction is blurred.
} 
Figure 3.1: Attendance rates to formal education (among Syrian refugee children 6-17 years old) in host communities and Zaatari Camp ${ }^{47}$

Currently enrolled
in formal education

Never attended formal education in Jordan

Dropped out of formal education

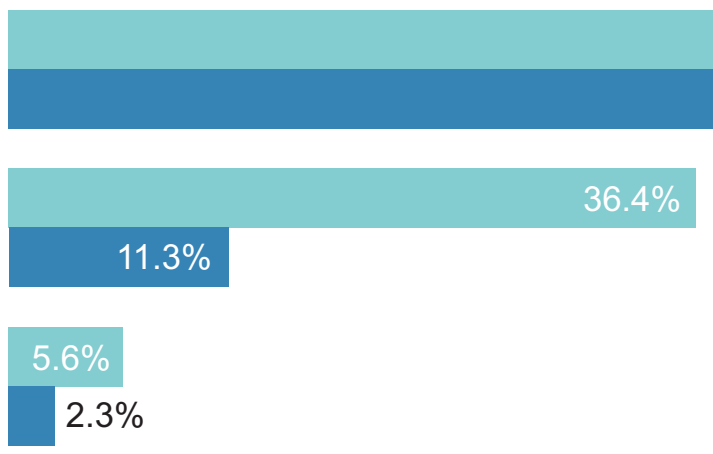

\section{$51.7 \%$}

\section{$63.8 \%$}

\section{Zaatari Camp \\ Host communities}

Data source: RAND's secondary analysis of JENA survey datasets

this. According to focus groups with children and parents, low attendance in Zaatari is associated with over-crowded schools, inexperienced teachers, lack of transportation within the camp, safety on the way to school in the camp, bullying at school, and lack of money for supplies. UN officials, teachers and NGOs also noted that the Zaatari schools are full; children are turned away. Furthermore, in focus groups with Syrian refugee children and parents in host communities, many explained that they did not register their children at school in Zaatari because they considered their stay at the camp to be temporary after entering Jordan, with plans to leave for a host community when possible.

Attendance also varies by children's family location in both host communities and camps, as Figure 3.2 illustrates. In host communities, attendance varies by governorate, with 49 per cent in Aqaba attending formal education, compared to 76 per cent in Karak. In Zaatari Camp, District 1 has the highest attendance rate to formal education (82 per cent) and District 4 the lowest attendance rate (33 per cent). The data therefore show that variations within settings appear to be more important than variations between them. Resources should be focused on areas with the lowest rates of attendance and further investigation carried out to identify the barriers to attendance specific to these areas.

\subsubsection{How access relates to education provision}

Multiple factors related to the effectiveness of the provision of education affect access.

\section{Enrolment policies keep children out of school}

According to stakeholder interviews and focus groups, enrolment policies are restrictive in two ways. First, enrolment into formal education is not allowed when a child has been out of school for three or more years. An estimated 65,000 of children and youth are ineligible for enrolment for formal education because of this policy. ${ }^{48}$ Furthermore, many children have never been to school before in any location; RAND's secondary analysis of JENA host communities survey data found that the top reason for both

47 As noted above (45) JENA survey questions asked about 'attendance', while describing it in a way that implied enrolment; no actual data about children's day-to-day attendance is available. Therefore in this chart this distinction is blurred.

48 Estimate from the JRP Refugee Pillar Needs Assessment 061014. 
Figure 3.2: Variation in attendance of Syrian refugee children in formal education by district in Zaatari Camp and by governorate in host communities

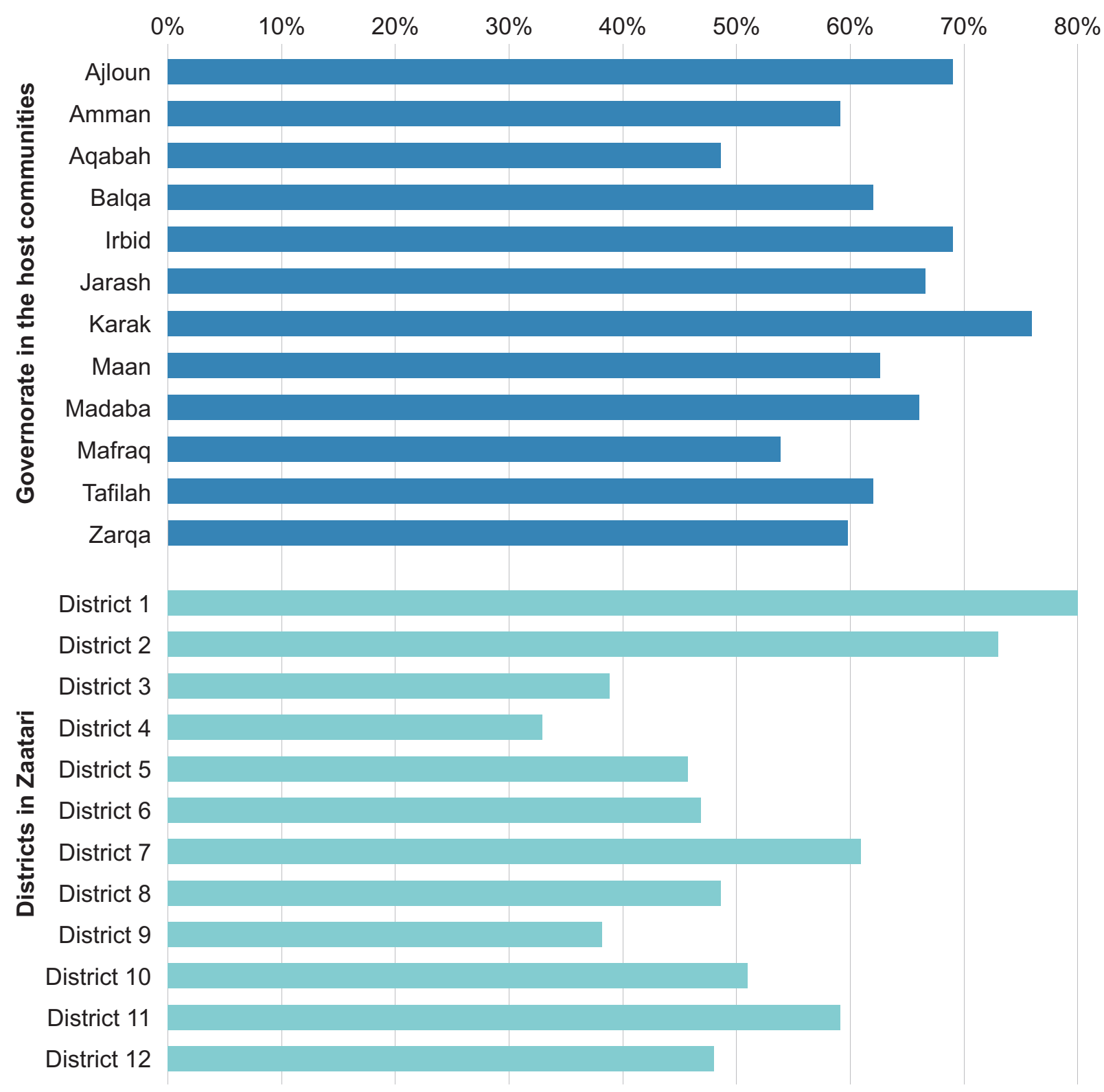

Data source: RAND's secondary analysis of JENA host communities and Zaatari survey datasets

girls and boys aged 6-11 being out of school was that they had never attended school before in any location in Syria or Jordan. There are no formal education programmes for young children (under the age of 13) outside of traditional ageappropriate groups. While there are alternative education programmes (discussed later in this chapter), these do not provide a full-time, certified education with pathways for younger children who cannot enter an age-appropriate formal classroom. Second, registration is not allowed on a rolling basis during the school year. When children arrive after the beginning of the school year, in some cases they must wait until the next year to enrol. According to focus groups and interviews, these policies and their implementation are not consistent. 


\section{Overcrowded schools lack spaces and materials}

Jordan's NRP noted that 8,000 Syrian children are on waiting lists for school places (not including the children ineligible for formal education because of MOE policy); schools cannot accommodate all of the children who need education. Host community survey data also shows that girls (aged 6-11) selected 'school is overcrowded and/or there is a lack of teaching materials' as their third main reason for never attending school in Jordan. Both girls and boys (aged 12-17) reported this as the fifth main reason for never attending school in Jordan. From the evaluation team's school visits, as well as comments from stakeholder interviews, it is clear that some schools are crowded while others are not. There are no data about this distribution, and UN stakeholders described a need for data in order to better plan expansion of school spaces.

\section{Lack of transportation to school inhibits attendance}

Some parents in focus group discussions noted that obstacles to access include distance to schools, children's safety and lack of transportation. In host communities, there is no public school transportation that could be used to take children to schools with available spaces. Some children in focus groups noted fears of safety during transportation to school (whether walking or other means). Survey data shows the main factor reported by 10 per cent of out-ofschool children in Zaatari for not attending was that school was too far away or not physically accessible. Some 13 per cent of children who no longer attend school in Zaatari reported the main factor for stopping was that school was too far away.

\section{Families question the quality of education and safety of the school environment}

Focus group findings from children and parents and survey data analysis highlighted quality of and safety in formal education as a factor affecting attendance. Some 9 per cent of children who have never attended school in Zaatari reported the main reason for this was because the quality of education was 'not good'; 6 per cent of children who dropped out of school in Zaatari noted this as the main reason why. Quality will be discussed in greater detail later in the chapter. Furthermore, children's sense of safety and well-being is compromised in some cases at school. As will be discussed in Chapter 5 , bullying in and near school as well as poor relations with teachers in some cases also reduces school attendance.

\section{Some parents lack awareness and information}

Although there have been outreach efforts to communicate with parents regarding their children's education options and requirements (in particular the Back-to-School Campaign, as well as information sessions in the camps), some parents in focus groups were not aware of education opportunities. While parents across all of the focus groups were able to identify two types of education options - provided by MOE and by NGOs - they were less informed about the choices available under each.

\subsubsection{How child and family circumstances affect access to formal education}

A number of factors related to child and family circumstances, and the wider social issues of the Syrians, affect access. Improving the effectiveness of the Programme to increase access to education requires understanding these factors and developing plans to address them when feasible; addressing many of these issues would require coordination with actors outside of the education sector.

\section{More recently arrived children have lower access}

The most recently arrived refugee children (from the last quarter of 2013 onwards) have significantly low formal school attendance (29 per cent), while over 70 per cent of children 


\section{"The school taught me and I}

beg of all families not to deprive

their children from getting their

education in this life and from

excelling and being happy."

Photograph and comment from a girl in Irbid

aged 6-11 who arrived before the end of 2012 were found to be in formal education. Based on interviews with a range of stakeholders, possible reasons are: full schools turn away additional new children; recently in a conflict zone, newly arrived children may be more traumatised; children with sporadic school attendance in Syria due to conflict may not be allowed to enrol in Jordan because of time out of school; or families may take time to settle in before sending children to school. In addition, as noted in the previous section some children who arrive during the school year may not be permitted to enrol until the following year. This delay may be reflected in the data and could also affect attendance more broadly.

\section{Older children are less likely to be enrolled in school than younger children}

In the host communities and Zaatari, both older boys and girls (aged 12-17) have lower enrolment rates than younger girls and boys, as shown in Figure 3.3. This may be associated with the pressure on children to work in order to assist their families, early marriage (for girls), or school safety. One in ten refugee boys and girls $(30,000)$ are estimated to be working as opposed to attending school. ${ }^{49}$

\section{Girls are more likely than boys to attend formal education, in both camps and host communities}

RAND's secondary analysis of JENA survey data highlights that girls aged $6-11$ have the highest enrolment rates across both settings (see Figure 3.3). Boys aged 12-17 have the lowest enrolment rates to formal school (49 per cent in host communities and 33 per cent in Zaatari). Focus groups and interviews with NGOs indicated that Syrian parents, pressured to support their families, may encourage boys 
Figure 3.3: Enrolment of Syrian girls and boys in host communities and Zaatari Camp

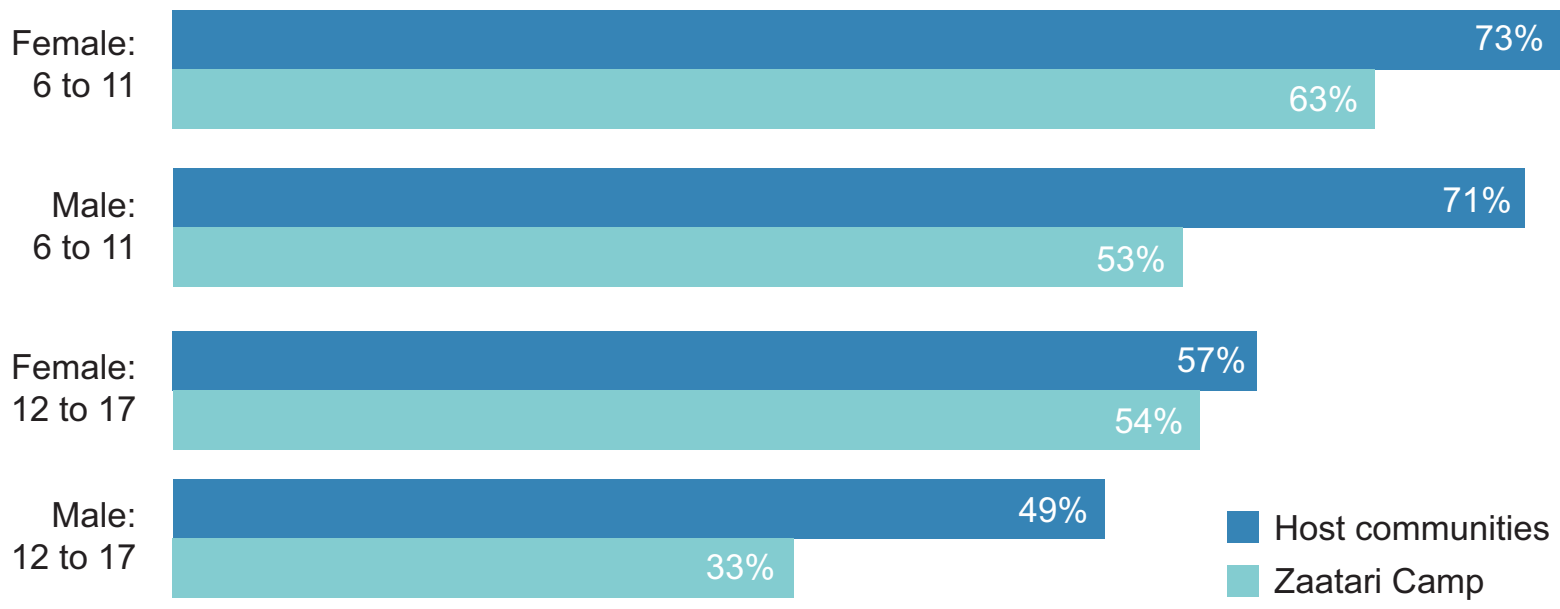

Data source: RAND's secondary analysis of JENA survey datasets

in particular to sell goods, beg, wait in queues to receive aid, and engage in building and cleaning work. Syrian girls are also engaged in these activities, but to a lesser extent. ${ }^{50}$ RAND's secondary analysis of the JENA host communities survey shows that need to work among boys aged $12-17$ is the most common reason given in host communities for never having attended formal education in Jordan. Further discussion of the gendered challenges, reasons behind them and recommendations can be found in Chapter 5 .

\section{Syrians not registered with UNHCR are less likely to attend school}

RAND's secondary analysis of JENA survey data shows this correlation. Interviewed UN officials, government officials and others assumed that unregistered Syrians might have more financial means or be in Jordan with work permits; therefore, programming was largely addressed toward registered refugees. Unregistered refugees could include Syrians who do not register as they do not expect to remain in Jordan for long enough, or who fear security or political repercussions. There could also be Syrians who do not know how to register or who cannot access a registration centre..$^{51}$ Unregistered Syrians are targeted with little or no humanitarian assistance and may be a vulnerable group that has fallen through the cracks of education.

\section{The level of education of the head of} household is an important predictor of attendance

RAND's secondary analysis of the host community JENA survey reveals that for host community households, 75 per cent of children whose parents had received a baccalaureate certificate or university-level education were attending education, compared with 53 per cent of children living in households where the head of household had not completed primary education.

\section{Costs of attending school are prohibitive for some families}

The top reason females aged $12-17$ gave in the JENA host community survey, for never attending formal schooling, was that they lack 


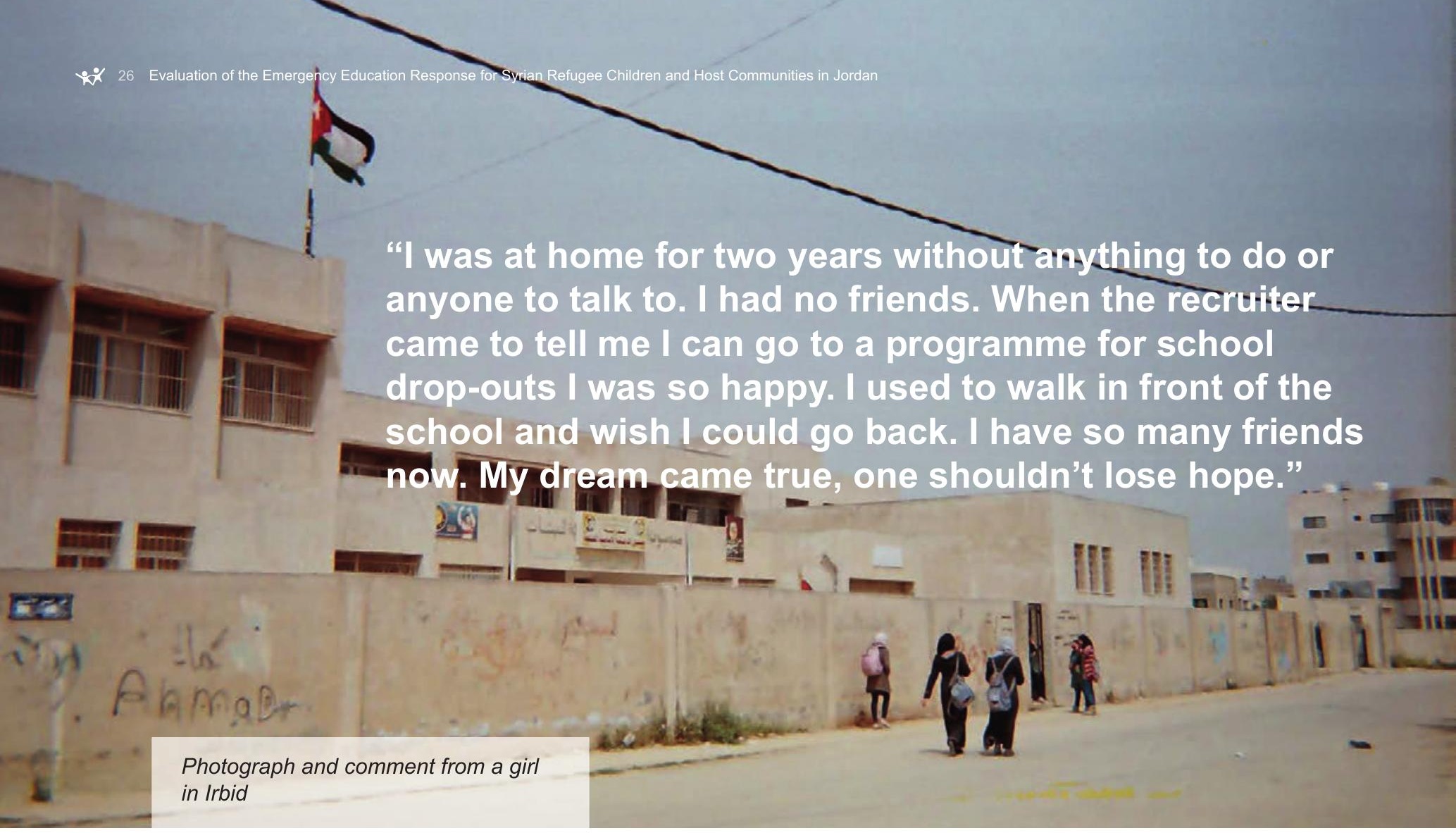

resources to spend on uniforms, shoes and books. This did not emerge as a key issue in the Zaatari data, perhaps because more is provided in the camps. A few children in focus groups also indicated costs, including transportation costs, as obstacles to formal education. At the same time, UNICEF and implementing partners provided 86,767 boys and girls with individual education kits (school bags and stationery) in the 2013-14 school year. ${ }^{52}$

\subsubsection{Effects on out-of-school children}

A key gap in effectiveness in meeting access goals is the large number of out-of-school children. These children will not have the education required to provide themselves with the skills needed as adults in Syria or Jordan, and the economies of both Jordan and Syria will be challenged by a generation of educationally impoverished people. As the UN 'Education For
All' goals ${ }^{53}$ note, education reduces poverty, promotes gender equality, reduces child mortality, and equips and empowers people with knowledge, skills and confidence to build better futures..$^{54}$ Children in focus groups who had been out of school and recently re-entered expressed gratitude for the structure and meaning that school gave them.

Furthermore, being in school is protective for children both physically and psychologically; without it children are at risk 'of losing hope, or accepting violence as normal and replicating it', which could fundamentally undermine their futures and the future of their nations. ${ }^{55}$ The potential for mutually reinforcing benefits from integrating child protection and education services is clear, and this was recognised across a broad range of stakeholder interviews. The daily structure provided by education can bring psychosocial benefits in itself. In this sense, the

52 Emergency Education Response, UNICEF Key Figures - June 2014.

53 UN. (2015)

54 UNESCO (2011).

$55 \quad$ No Lost Generation (2014) 
access barriers are an acute protection concern, reinforcing how important it is to children's development and protection to be in a school environment.

Significant data gaps impede planning. While at least 97,000 children are not attending formal education, the actual numbers of out-of-school children, their locations and their needs are unknown because there are no clear numbers of unregistered refugees, and because it is not clear what percentage of enrolled children are actually attending school on a regular basis. NGOs that set up focus groups for the evaluation team found it very difficult to reach these children. The implementation by UNESCO of the Education Management Information System (EMIS) should allow progress in relation to some aspects of this problem.

It must also be noted that further to the numbers of Syrian children who are out of school, there are an estimated 31,000 Jordanian children of primary and lower secondary ages who are in the same situation. ${ }^{66} \mathrm{It}$ will be important to also focus on the challenge of out-of-school Jordanian children.

Syrian refugee children out of school in other countries will present further challenges to Syrian society in the coming years. By way of comparison, in Turkey 2013 government figures put school attendance by 6-11 year olds outside camps at only 14 per cent ${ }^{57}$ and in Lebanon it is estimated that more than 50 per cent of Syrian refugee children aged 5-17 are excluded from any form of education. ${ }^{58}$

\subsection{Quality of formal education}

Quality education for the refugees depends in large part upon the overall quality of the Jordanian formal school system. Jordan had a robust education system that until recently had been steadily improving over the previous few decades. ${ }^{59,60}$ In 2003, Jordan initiated a programme to improve quality through its $\mathrm{K}-12$ education system, entitled 'Education Reform for Knowledge Economy' or ERfKE. ${ }^{61}$ ERfKE entailed teacher training, reducing double-shift schools and a new emphasis on English and information skills. Jordan has achieved near universal primary education. ${ }^{62}$ However, even with these improvements, Jordan's education system still faces a number of challenges, including school crowding, student attainment and teacher training. Jordan is one of the few countries of the Middle East to participate in the Trends in International Mathematics and Science Study (TIMSS), an international exam established to compare educational performance among countries. Jordan scored below the TIMSS scale centrepoint, placing 33 out of 36 countries. ${ }^{63}$ However, most countries participating are OECD countries, and participation alone is a sign of making efforts towards improving educational quality.

During Jordan's education response, quality took secondary priority to access. As one UN official noted, 'What's happened here has been what happens everywhere: first access then quality.' As the Programme makes efforts to transition from an emergency to a resilience approach, a focus on quality has not yet been realised. Yet, recognising the underlying strengths of Jordan's

\footnotetext{
56 Middle East And North Africa Out-Of-School Children Initiative and UNICEF (2014).

57 AFAD (2013).

58 UNHCR (2014c).

59 Chapman (2011).

60 The Millennium Development Goals Report (MDGR) for Jordan (2004).

61 World Bank (2010).

62 UNESCO Institute for Statistics (2005).

63 National Center for Education Statistics (2012).
} 
education system observable before 2011, the quality of education that could be delivered for the Syrian refugees in Jordan is potentially good.

\subsubsection{Multiple factors affect the quality of formal education for refugees}

This section analyses how effectively the education response meets the quality objectives of formal education for refugees and describes efforts underway to improve quality. A quality framework establishing minimum standards has been developed with the Inter-Agency Network for Emergencies (INEE), ${ }^{64}$ and by agreement with the ESWG. However, this has not yet had time to become established.

\section{Quality of formal education in host communities and camps is perceived as relatively good}

Only 9 per cent of respondents in host communities and 20 per cent in Zaatari described the quality of education as bad or very bad, according to RAND analysis of JENA data. In Zaatari, 37 per cent of respondents reported that education had improved in the previous year. However, as discussed in the rest of this section data from focus groups and interviews with a range of stakeholders point to significant problems with perceived quality.

\section{Student learning among the Syrian refugees is sub-standard, as reflected by low test scores}

Outcomes for Syrians in Zaatari taking Jordan's secondary school exit exam, the Tawjihi, were very poor: only 2 per cent of the Syrians taking the exam in Zaatari passed according to an interview with a UN official. Data on the pass rates of Syrians in host communities were not available for this evaluation. In comparison, the pass rate among Jordanian students is approximately 40 per cent. ${ }^{65}$ While it is expected that children who have studied the Syrian curricula would not do as well in Jordanian examinations as students who studied Jordanian curricula, the difference in pass rate levels is indicative that student learning is not as desired.

\section{Class sizes are large and classrooms are crowded}

Opening up Jordanian schools to large numbers of Syrians has resulted in overcrowded classrooms, compromising quality instruction. In some of the focus groups, teachers reported classes of over 50 students. UNICEF (2014) figures show a student-teacher ratio in camp schools of 1:70-90 (average). ${ }^{66}$ Teachers and children were concerned about class sizes consistently across the majority of focus groups.

\section{Teachers struggle in difficult school environments}

Teachers in focus groups reported that they lack the skills and resources necessary to manage the circumstances they face: a diverse, overcrowded classroom; children with psychosocial and behavioural problems or special needs; students of different educational levels and ages; and inadequate infrastructure. Teachers indicated that they are unable to give students enough attention and manage the classroom, which was substantiated by the children's focus groups and interviews with UN officials and NGOs. It was noted in focus groups with both teachers and children that teachers used corporal punishment to control large class sizes; teachers indicated that they do not know how else to handle classroom management in the situations that they face. According to focus groups, corporal punishment was used with students irrespective of nationality.

UNICEF's figures show that 2,100 teachers were trained in $2014 .{ }^{67}$ In focus groups, some teachers

\footnotetext{
64 INEE. (2016)

65 MOE.

66 From UNICEF Education Section Analysis, March 2014.

67 Data gathered from the UNICEF and CADAR report.
} 
"I don't like this about school, they have left all the broken chairs. I don't like the chairs we have now because the ones we have are broken."
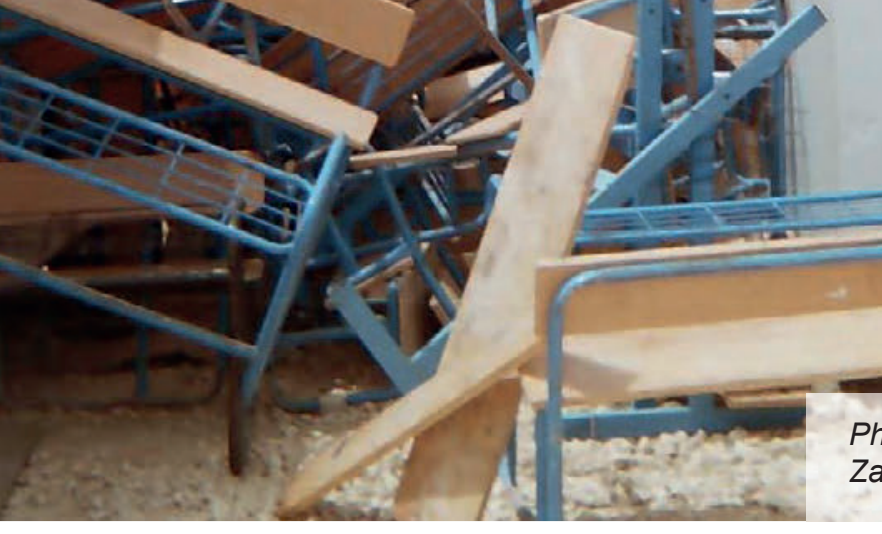

Photograph and comment from a girl in Zaatari

reported receiving training on pedagogy and strategies for dealing with a traumatised student population, but generally regarded the training as insufficient. Some teachers reported the need for training in handling students with behavioural problems and special needs, especially within the context of large classes. UNICEF stakeholders reported that a teacher mentoring initiative in camps was reported to be effective, but also too resource-intensive to maintain.

\section{Syrian assistant teachers in camps add significant value}

According to GOJ policy, employment is not legal for most Syrians in Jordan and in accordance with this, Syrians are not permitted to be hired as teachers. However, they can work as assistants to Jordanian teachers in camp schools. Syrian assistant teachers add significant value according to school staff, NGO and UN stakeholders, and focus groups with children, who reported that as well as bringing down the teacher to student ratio, the Syrian teachers could explain lessons in a way that was more familiar to the students. The presence of assistant teachers has also allowed follow up of children's homework, and camp school principals reported that parents' appreciation of this has directly helped to keep children in school. Assistant teachers also helped to reduce school bullying and violence. Some Syrian teachers in focus groups reported good relations with Jordanian teachers. However, others felt marginalised and that their presence was not fully utilised, because of tension when Syrian assistant teachers were more experienced than Jordanian teachers.

\section{Learning is affected by poor facilities}

In both focus groups and stakeholder interviews, it was noted that the school environment and facilities available in formal schools in both camps and host communities hamper the quality of formal education. Schools in Azraq lack electricity and air conditioning/heat, and teachers in Zaatari also commented that electricity cuts and lack of heating and lighting impact on students' achievement. According to one student in Azraq Camp, 'we melt in the heat and we are cold during the winter, but we come.' Teachers in Zaatari spoke about teaching computer science theoretically because they lack computer labs, electricity and printing facilities. In focus groups in Amman, participants spoke of rubbish in classrooms. Students in host communities also suffer from water shortages at school. Furthermore, one focus group in Mafraq spoke 


\section{"It is like a prison not a school."}

Photograph and comment from a girl in Zaatari

about the playground spaces being small and shared with children of all ages.

The lack of appropriate playground spaces was also mentioned by a principal in Zaatari. As one principal noted, 'The tools needed for success are unavailable.' UNICEF's provision of 69 schools with furniture and equipment, and 65 schools with prefab classrooms is an example of effective practice, providing resources for the refugees while also leaving Jordan with benefit. ${ }^{69}$

\section{Student engagement is affected by multiple factors}

Sustained engagement of children in their classes throughout the school year is affected by a range of factors. Some teachers during focus groups reported that attendance is high at the beginning of the school year and dwindles as the year progresses. Several teachers described how some students do not attend classes and just show up to the exams. According to focus groups with teachers, parents and children, factors affecting sustained student engagement include teacher skill and treatment of students, class size, school environment, difficulty adapting to the curriculum, fear over economic circumstances, the disruption that comes from frequent changes in accommodation, and putting children with wide age gaps together in a classroom.

\section{Differences in curriculum and certification make it difficult for students and teachers to adapt}

A wide range of stakeholder interviewees felt that differences in the Jordanian and Syrian curricula make it challenging for some Syrian students, in particular secondary students, to enter Jordanian schools. According to focus groups with teachers and children, differences in curricula, however,

68 It is worth noting that while children perceive the barb wire fencing negatively, making school feel like a prison, UNICEF representatives explain that such fencing contributes to the children's safety and to the security of the school environment. Such fencing also helps to prevent domestic and external vandalism. 

Table 3.4: Double-shifted schools for Syrians by location

\begin{tabular}{|c|c|c|c|c|c|c|c|}
\hline Amman & Irbid & Madaba & Zarqa & Ajloun & Jarash & Mafraq & Camps \\
\hline 27 & 45 & 2 & 9 & 2 & 2 & 11 & $\begin{array}{l}\text { Total }^{70}: 20 \\
\text { Zaatari: } 12 \\
\text { Azraq: } 4 \\
\text { EJC: } 4\end{array}$ \\
\hline
\end{tabular}

\subsubsection{Double shifts work less well in Jordan than elsewhere}

As shown in Table 3.4, expanding double shifts has been an effective approach towards rapidly increasing Syrian access and school capacity; double-shifted schools in host communities and camps have provided school spaces for about 70,000 Syrian children.

Double shifts in Jordan pre-existed the refugee crisis, and many Jordanian students attend double-shift schools. In 2012-2013, there were 232,000 Jordanian children (7.6 per cent of students) in 426 double-shifted schools, ${ }^{71}$ out of 3,555 schools in Jordan. In 2014, the MOE created 98 double shifts for Syrian children in host communities and 20 double-shift schools in camps, according to MOE data provided for this evaluation, distributed as shown in Table 3.4. The proportion of students in double-shifted schools in Jordan rose from 7.6 per cent in 2009 to 13.4 per cent in $2014 .{ }^{72}$ Reliance on double shifts has increased pressure to ensure quality is maintained.

Double-shifting has been effective in promoting access and quality in education in several ways:

- Double shifts reduced crowding. In some schools that the evaluation team visited, second shifts for the Syrians were less crowded than first shifts for the Jordanians.

- Double shifts reduced pressure on both communities in the short term. Some
Syrian parents and children in focus groups expressed a preference for separate Syrian shifts, as it enabled attention to their needs as Syrians (catching up to the curriculum, dealing with the trauma that the Syrians share) and reduced bullying. According to interviews, Jordanian parents also pressed for separation, as some viewed the changed classroom conditions as disruptive for their children.

However, there are ways that double shifts have reduced quality, for both Jordanians and Syrians:

- Double shifts reduced instructional time. In double shifts lessons are 30 minutes long, whereas in single shifts they last for 45 minutes. In focus groups, some teachers and students said that they struggle with this reduced instructional time. When coupled with large class sizes, the shortened lesson times become an even bigger problem. Double-shifting to accommodate the Syrians means that the Jordanian students in the first shift also have reduced instructional time.

- Teachers for Syrian-only second shifts lack experience. Teachers for the second shifts are Jordanian university graduates hired from civil service waiting lists. UN and NGO interviewees expressed concerns related to the inexperience and lack of motivation of these teachers and the quality of their teaching, in particular in the context of teaching a refugee population.

70 Excluding the annex in Zaatari.

71 MOPIC (2014b).

72 MOPIC (2014b). 
- Second-shift teachers are paid less than first- or single-shift teachers and not on time. Teachers hired in order to expand capacity in response to the Syrian influx, noted in school visits and focus groups that they were not hired under the same terms as other teachers, getting paid less or not on time. Second-shift teachers in host communities said they are paid 200JD per month, instead of 400JD per month like other teachers. In camp schools, UNICEF supplements incomes, but salaries still do not equal those of first- or single-shift teachers.

- The afternoon shift for Syrians is deprioritised in terms of resources, and in some cases, reduces resources for host community children in first shifts. In several schools visited for the evaluation, school administrators said that second-shift students lack resources that are available in the morning, such as librarians, computer and science labs, and cleaners. In a few focus groups with teachers, it was also noted that because of time limitations, the first shifts for the host communities had cut art, vocational training and physical education classes.

- There is little school monitoring of the second shifts. According to interviews with NGOs and UN officials, MOE school monitoring is less in double-shifted and camp schools than in other schools, while being more necessary, as the second-shift teachers often lack experience.

- Segregated double shifts raise concerns about social cohesion. UN officials and donors raised concerns that double-shifting with segregation by nationality will create longer-term risks to social cohesion, with Jordanians and Syrians educated in separate and unequal schools. Separation into morning and afternoon shifts creates two schools that barely mix, limiting the scope for longer-term building of mutual understanding and trust.

A number of studies have shown that doubleshifted schools in other countries facing constrained school infrastructure can produce results similar to single-shifts. ${ }^{73}$ For example, at different points in their development, South Korea, Singapore, Indonesia, Hong Kong and Brazil have all relied on double shifts. Another example is the UNRWA schools in Jordan; despite being double-shifted, they outperform average schools in Jordan and indeed scored above the world average on international tests, while using the Jordanian curriculum. ${ }^{74}$ The keys to quality results in double-shifted schools in other circumstances are several. First is equal and adequate instructional time, achievable, for example, by increasing the number of school days to compensate for shorter daily lessons. The OECD average is 800 hours of instructional time per year, and Jordanian single-shift schools approximate this amount of time. ${ }^{75}$ Another key to quality is equivalent teachers and resources provided for both shifts. It is also important to note that in the above cited examples, students were not separated in double shifts by minority or refugee status.

\subsubsection{Meeting the need for quality education among vulnerable Jordanians}

As voiced across a range of stakeholder interviews, accommodating so many additional students quickly with constrained resources endangers Jordan's own goals for improving quality of education for its citizens. One UN stakeholder put it, 'The more Syrians we put into formal, the more impact on Jordanians.' Interviews outlined several key challenges to effectively addressing quality for Jordanians. 
Donor funding for Jordan's Education Reform for a Knowledge Economy (ErfKE) has slowed, while it was a key focus of international donors in Jordan prior to the refugee crisis. In particular, diverted resources hamper abilities to address the 31,000 out-of-school Jordanian children, reduce the number of double-shifted schools in Jordan, renovate aging school infrastructure, train teachers, and continue to upgrade the curriculum. Financing the expansion of the school system has become a major concern for the GOJ.

Furthermore, a wide range of stakeholders noted that classroom conditions have worsened for vulnerable Jordanians because of strained facilities, classroom crowding, reduced instructional time from double shifts, and slower presentation of the curriculum to accommodate new children adapting to Jordan's curriculum. In northern governorates with many refugees, infrastructure is reportedly especially strained. Of the 3,555 MOE schools in Jordan, ${ }^{76} 41$ per cent were already overcrowded in 2013. ${ }^{77}$ While data were not available for this evaluation about current rates of overcrowding, with the addition of the Syrian students crowded conditions are likely to have worsened since then.

In this context social tensions between Syrians and Jordanian host communities are an ongoing concern. Focus groups with teachers, parents and children showed variation about this issue, with some describing warm and positive relations and others describing worsening relations and discrimination. Interviews with teachers and school management offered promising examples of social cohesion, such as community- and school-led initiatives to support Syrians in need. Some schools in host communities reported reduced tensions between Syrian and other students over time. In school visits, some
Jordanian teachers and principals described how they emphasised to their students the need for friendship and solidarity, explaining the Syrian situation and combating negative preconceptions.

\subsection{Access, quality and strategy of alternative education}

To meet the needs of Syrian and Jordanian children and support the provision of formal education, the EER sponsored a number of alternative education programmes. The rationales for providing alternative education programmes are several. Based on interviews with stakeholders, the documented agreements between UNICEF and NGO implementing partners, and the ESWG glossary, this evaluation broadly categorises alternative education programmes as (1) non-formal programmes that support formal education, and (2) informal programmes that are supposed to support children who are not (yet) accepted into the formal system or who are on waiting lists to enter the formal system. At the same time, some interviewees expressed an assumption that these alternative programmes would have to replace formal education for children who could not be accepted in formal schools due to MOE policy.

Non-formal education was initially developed as a programme for Jordanian school-leavers who had been out of school for three years or more, with secondary certification for students ages 12 years and up. Non-formal education for Syrians is provided though drop-out educating programmes, home-schooling programmes, evening studies and summer studies programmes.

All other educational programmes are classified as informal. These are not certified by the MOE and are not targeted to certain age groups. ${ }^{78}$ Such activities include catch-up support to enter 


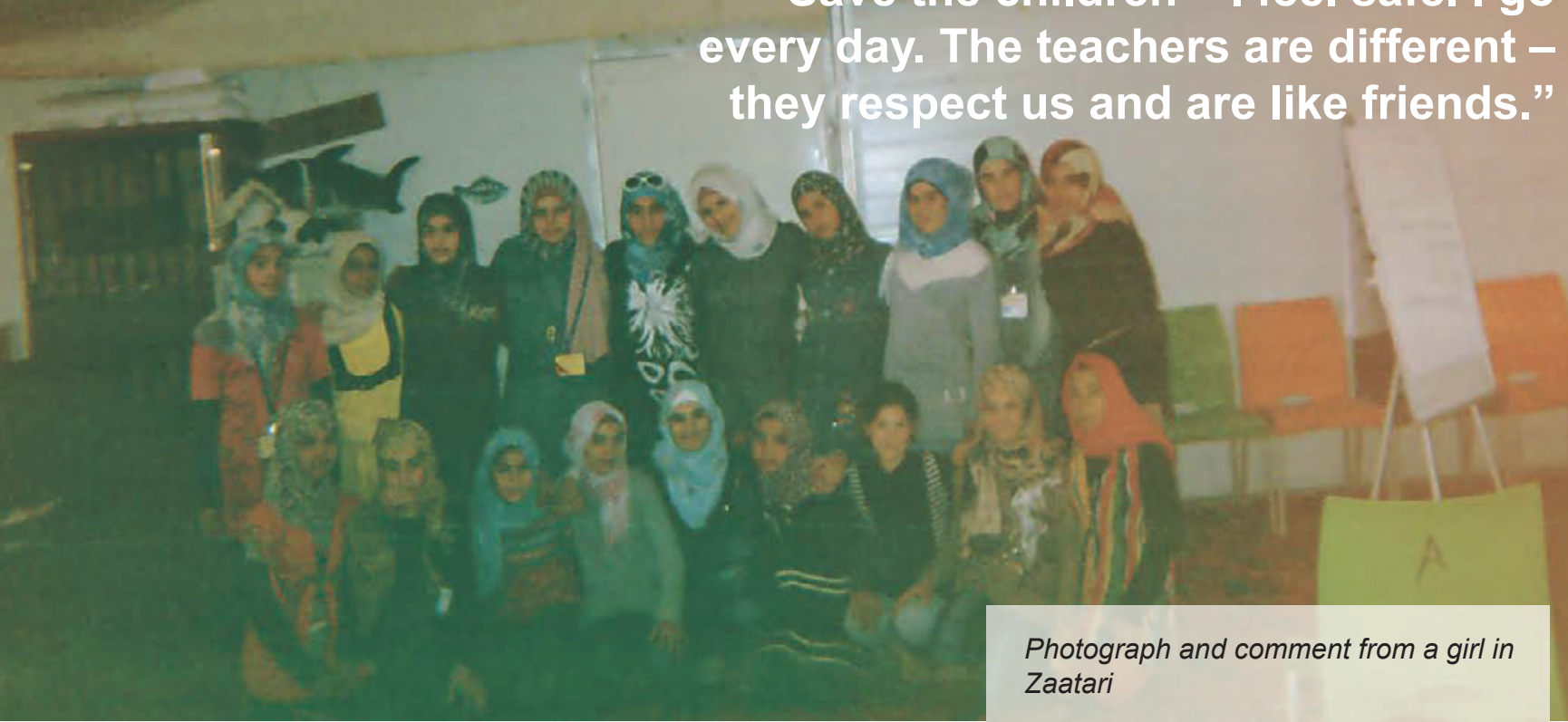

into a formal school, supplemental programmes (holistic recreation, life-skills support, education mixed with psychosocial support, extra support for children with disabilities), programmes for child labourers who lack access to formal education, and Makani integrated child-friendly centres. Makani centres are a comprehensive approach to service provision, which provide services covering informal education, skills building programmes and psychosocial support all in one site for Jordanian and Syrian communities to access.

Outside of these systems is religious education. Some families prefer to send their children to mosque-based programmes for reasons related to values, lack of spaces in the formal system, or protection or quality concerns with the formal system. This evaluation did not cover religious education programmes, and there is little documented evidence or data about their extent or content.

\subsubsection{Alternative programmes have greatest reach in camps}

Access to alternative education is higher in camps than in host communities according to RAND analysis of JENA survey data. In the host communities, 2 per cent of school-aged children attend non-formal education ${ }^{79}$ and 3 per cent of school-aged children attend informal education; in Zaatari, 21 per cent of children attend informal education. In Zaatari, attendance to both nonformal and informal education programmes is lowest for boys aged 12-17 years; highest attendance is for girls aged 6-11 to informal education programmes. Lower attendance in host communities may be because of disproportionate donor, UN and implementing partner attention

79 In UNICEF (2013a), definitions for non-formal and informal education in host communities were not well understood by enumerators, so there may be errors in these figures. Note that data for the Joint Education Needs Assessment Report was collected separately and not as part of this evaluation. 
to camps. A range of stakeholder interviewees noted it might also result from difficulties in identifying students, a lack of outreach in host communities to raise awareness of such programmes, and shortage of transportation. Informal education partners often rely on referral pathways from other organisations to identify children for attendance. These systems may be stronger and easier to manage in camps than in host communities. Differences could also be because more children in host communities are attending formal education and do not need the alternative education.

\subsubsection{Alternative education is perceived to be high quality}

Alternative education programmes have been perceived as effective in providing quality. Many NGO and UN stakeholders spoke enthusiastically of the role and quality of alternative pathways, highlighting their potential to raise standards across the education system by acting as an 'innovation bed'. Implementing partners emphasised a role for alternative pathways beyond their function as an 'overflow' for the formal system and saw informal education as offering more a relevant, child-centred and flexible education to Syrians. This idea is supported by reports of some students dropping out of formal schooling in favour of informal classes. As one implementing partner declared: '[we've] really achieved results we didn't think we could'.

Perception of the quality of alternative education is also high among Syrian parents and children in focus groups. ${ }^{80}$ Syrian parents and children in focus groups also viewed informal/non-formal teachers ${ }^{81}$ as attentive to student needs. Analysis of focus groups and interviews indicates that it may be because:

- Programmes were designed for Syrian refugees
- Lessons are less hurried

- Students may be taught at their level, without artificial placement based on age

- Teachers were hired to have the needed skills

- Many teachers are Syrians, and Syrian children respond favourably to them

- Classrooms are smaller and teachers have less stress in the classroom

- Teachers are paid more than in second shifts

- Teachers do not use corporal punishment and treat children with respect

- More teachers have training in helping children with behavioural or psychosocial problems

- Teachers revisit lessons to ensure understanding.

Very few parent focus groups raised concerns regarding the quality of alternative education, though some did question the short duration of the programmes and their sustainability. Others reported children only attended three times a week, and several programmes faced funding challenges. Some service providers at the camps indicated that lack of knowledge and shortages in resources made it challenging to align their teaching well to the MOE curriculum and Tawjihi and to facilitate student transition to MOE schools or higher education. Service providers mentioned that getting skilled teachers was a challenge, and also cited a lack of learning tools (e.g. computers and trainers) as a key barrier to quality.

Makani centres are an example of innovation in alternative education. By expanding existing child friendly spaces nationwide to become alternative learning centres, UNICEF and partners aim to offer integrated and cost-effective spaces to deliver education and protection interventions to Syrian and Jordan children

80 However, it is worth noting that parents may not readily distinguish between the different forms of education. This might have implications for the awareness raising campaigns that UNICEF partners are undertaking.

81 Note that in the focus groups, parents and children were not able to distinguish between informal and non-formal programmes. 


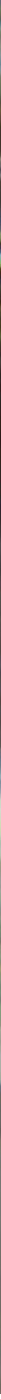

and youth as well as community outreach activities. Makani centres were developed based on lessons learned from previous poorly coordinated and expensive sector interventions. ${ }^{82}$ The inclusive approach also offers a pathway to community ownership and sustainability. As one official noted during an interview, 'Our ultimate goal is for the community to get the keys.' The effort to include both communities is seen to be a challenge, but centres generally reported a good balance in the numbers of Syrians and Jordanians using their services. As UNICEF sources have indicated, this model offers a promising platform for inclusive work on antiviolence and trust-building in the future as local communities become more engaged. Given the generally positive experiences of informal education among parents and students, the initial expectations of partners, and the logic of linking a range of education services with other services in the community, there are good reasons for being hopeful about the success of Makani centres. However, it is too early to evaluate their effectiveness.

\subsubsection{Alternative programmes lack clear standards, certification, adequate instructional time and pathways}

There remain concerns about the relevance, sustainability and quality of alternative programmes. While evaluators have been told of good individual alternative programmes, as a whole they are not coordinated to meet clear goals and quality standards, or developed enough to provide certification and pathways to students. Most are not set up to substitute for a certified, full-time education, but rather 
"The picture is about an activity we had in the Center. We watch movies as a tool for learning about different topics. The movie referred to in this photo is about peer pressure... and particularly about a character in the movie, who guided children to stay away from bad friends. We learned through this activity about respect, and how to respect others in the Center. Many times, we watch short movies and discuss the ideas behind them. We learn a lot here, I like to come to the Center."

are supplements. There is great potential for the alternative system, but it needs improved strategic coherence. Filling children's time with learning activities is not enough.

To meet Syrian and Jordanian children's needs now and into the future, visible and viable pathways are needed to next steps in education and on to adulthood. A range of stakeholders, including NGO and UN representatives, noted that links enabling children to move from alternative education into formal education are often missing; when many children finish alternative education, they find that there are no places for them in formal education. According to these stakeholders, the primary challenge to quality provision in alternative pathways was coordination on standards and a unified, certified curriculum. Families want an education that will lead to certified pathways of continued and completed education and certification for the workforce; the certification gap is a major concern for parents and students, producing distrust in alternative education services. Their concerns stemmed from the following factors:
- Completing an informal programme does not guarantee a place in a formal classroom because of lack of school spaces.

- Completing an informal programme does not lead to certification.

- Some certifications could lead to a protection risk upon return to Syria as they would identify Syrians as having been refugees, with possible stigmatisation.

- Syrian teachers in alternative programmes lack training in the Jordanian curriculum.

- Parallel certification structures and multiple uncoordinated programmes create incoherence.

The development and roll-out of a unified informal curriculum has been a recent focus of UNICEF, and they have also worked with the MOE to issue letters of equivalency which officially acknowledge achievements in alternative education. Indeed, while some alternative learning centres reported that their remedial education used the MOE curriculum, 
developed in collaboration with UNICEF, other NGOs reported that they were not using any curriculum at all.

The lack of a clear framework for what alternative education is intended to achieve over the longer term and inconsistent monitoring of quality means that standards and achievements are hard to measure. Work on quality indicators has developed somewhat, with inclusion of a new indicator on how many children return to formal school from alternative pathways. However, according to a UN interviewee, M\&E is not yet as comprehensive as required. While some donors conduct monitoring visits to track quality, this is ad hoc and not systematic enough to feed into system improvements. A range of stakeholders from schools, NGOs and the UN reported that a lack of focus on learning achievements, drop-outs and grade progression is a major gap.

Furthermore, there are no available data to compare instructional time across alternative programmes, or between alternative programmes and formal education, as discussed in Chapter 4. While that may have been an appropriate state of affairs during the worst of the crisis, several years in the absence of a full-time, certified pathway to education, with instructional time in accordance with international averages ( 800 hours per year, as the OECD average), ${ }^{83}$ is a major gap.

With regard to non-formal education in particular, NGO and UN stakeholders considered it valuable for eligible Syrians but highlighted key gaps. Non-formal education caters only to those aged 12 years old and up. This is a particularly big gap given that 65,000 children are estimated to be out of school because they have been out of school too long to enter formal classrooms. As above, the primary reason in the JENA survey data for children aged $6-11$ to be out of school was because they had never been enrolled in school anywhere; because of that, they are not eligible for enrolment. According to interviews with UNICEF staff, the extension of non-formal activities to younger students and the development of an age appropriate non-formal system that caters to their needs is an advocacy focus of UNICEF.

\subsection{Summary and conclusions}

In the simplest terms, the Programme's key objective is to provide access to quality education for all children affected by the crisis. With about 130,000 Syrian children enrolled in formal education and 35,000 attending alternative education programmes, the education response has effectively achieved a significant level of access to education and supported formal education with alternative approaches. However, the large numbers of children (at least 97,000 ) who are out of formal school demand a renewed strategic response.

Improving the effectiveness of providing access requires addressing the multiple determinants of access: these are associated with location, family and child circumstances, and provision of education. Factors related to location and educational provision can be addressed through educational policy and programme changes. Factors related to the family and child (such as child labour and early marriage) may require a multi-sectoral approach, which would depend on UNICEF, donors and other actors pooling their advocacy capabilities to prioritise and work towards solutions. Because the number of outof-school children is such an acute problem, this evaluation spent significant time on the analysis of access to education and potential solutions, as described in Chapter 7.

As with any emergency response programme, the initial focus of the EER was on facilitating 
access to services. Once progress has been made it is important to ensure that there is also a parallel emphasis on quality of provision. There remain many challenges in effectively meeting objectives of quality education. Problems with quality can be seen in crowded classrooms, overwhelmed teachers, facilities in need of upgrade and repair, and low test scores. Furthermore, with the influx of Syrian refugees into the Jordanian formal system, the quality of education provision for Jordanians as well as for Syrians has become strained.

In the absence of access to schools for all, to meet some of the needs of the Syrian and
Jordanian children UNICEF and donors have sponsored a number of alternative education programmes. Such alternative programmes have greatest reach in camps, rather than in host communities, and are considered to offer innovative, quality approaches and activities to children. Alternative approaches that draw on international expertise could help to help fill access gaps, provided that they are structured around a coherent strategy with quality standards and pathways to adulthood. This would require provision of a full-time certified alternative education as cost-efficiently as formal schooling. 


\section{Findings: efficiency}

The DAC criteria describe efficiency in development efforts as when 'aid uses the least costly resources possible in order to achieve the desired results.' In the case of the Emergency Education Response Programme, this involves achieving both allocative efficiency (delivering goods and services that accurately reflect the balance of need) and productive efficiency (producing goods and services to the required standard for the lowest cost). To assess allocative efficiency, this evaluation therefore asks whether the Programme has in place a process for ensuring a balanced portfolio of activities based upon the available evidence of needs and available resources. The absence of such a process would be evidence that the Programme is unlikely to be optimally balanced. As a leading agency in the Programme, with a key coordinating role, UNICEF has particular responsibility for ensuring that this process is in place within a national framework primarily determined by the GOJ. To review productive efficiency, key evaluation questions are whether cost data are available and used to drive more efficient delivery and whether outcome data are used systematically to identify efficient delivery and spread effective practice. The absence of evidence that this is happening would in itself suggest that productive efficiency was probably not being achieved. Once again, it might be expected that UNICEF would play a key role in identifying costs and identifying and promoting efficient practice. Finally, achieving efficiency is a process of constant improvement, depending upon quality monitoring and experiential learning.

This chapter outlines an analytical framework within which to discuss the findings about efficiency, shown in Figure 4.1, as the EER does not have an existing framework for efficiency.

The pursuit of efficiency in Figure 4.1 is a continuing cycle of activities with each stage contributing to overall efficiency: identifying

Figure 4.1: The EER cycle of delivering efficient services

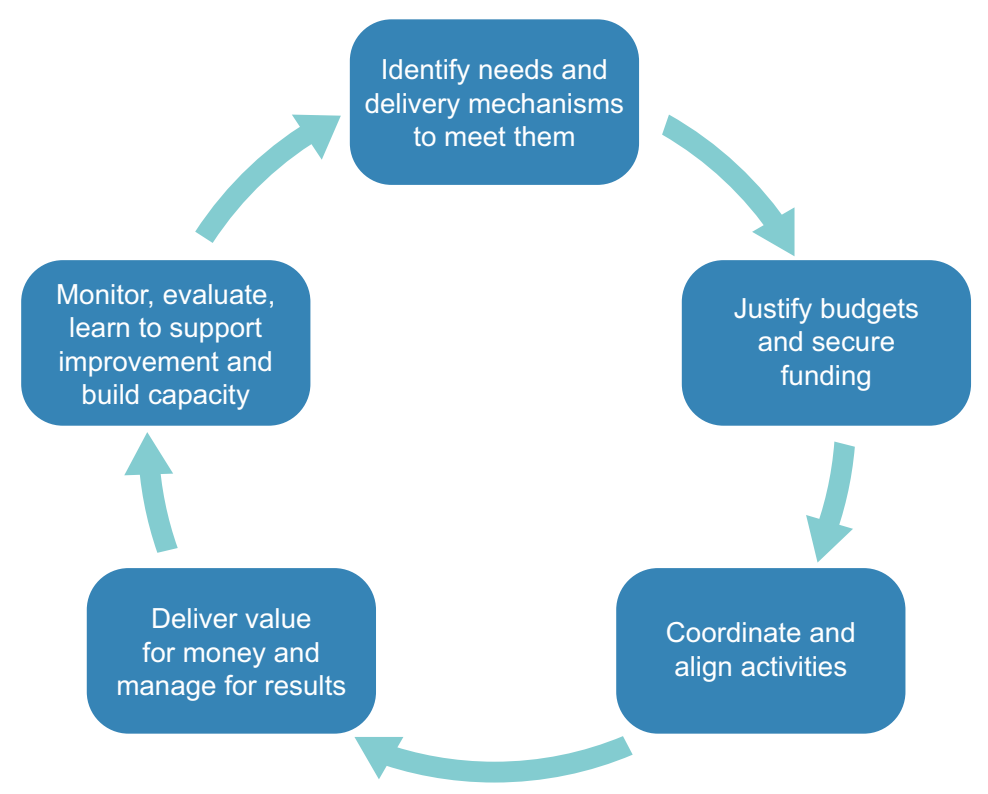


Table 4.1: Efficiency - overview of evaluation questions and findings

Evaluation question
Identifying needs and
developing services to
address them, including
how efforts were designed
to meet learning needs
assessments

Justifying budgets and securing funding

Coordinating and aligning activities

Delivering outcomesfocused management

Using monitoring and evaluation to drive improvement and build capacity

Procurement policies, controlling costs and

\section{Overview of findings}

Efficient allocation of resources within the Programme is linked to the identification of needs. Data were often relevant, sufficient and credible. However, further improvements are required to ensure that allocation of resources are prioritised to reflect the distribution of needs. Additionally, there appears to be no systematic options appraisal to ensure that the activities are designed to meet needs as efficiently as possible. There were no learning needs assessments available for the Syrians in Jordan.

Planning tools, as well as fund-raising tools, such as the RRP6 and 3RP, NRP 20142016 and JRP 2015 are limited by several factors including: cynicism regarding the amounts of funding requested, the view that it was not prioritised, and perceived lack of transparency for donors. Planning documents could be strengthened to mobilise funding and create a transparent account to donors. Donors, in response, should consider stable long-term funding to support longer-term investment in efficiencies.

UNICEF has played a positive role in supporting the ESWG at the sectoral level, and the GOJ at the national level, in achieving alignment of specific activities with overall strategic goals. The ESWG requires further support and re-energising while tensions remain between IPs and MOE. Given the rapidly evolving situation this is understandable, but focusing coordination on efficiency improvements should be a high priority especially as the EER seeks greater focus on resilience and quality of provision. delivering value for money

Based on available data it is not possible to undertake cost effectiveness analysis; producing such data should be the focus of future efforts. Many components required to deliver value for money are either in place or being developed, and these should be brought together and communicated across the EER within an overall strategy. UNICEF should embrace a more analytical approach to identifying and communicating steps required to embed VfM within existing activities. UNICEF lacks the data needed to demonstrate that the EER delivers value for money.

Overall, the relationship between UNICEF and implementing partners has been focused on transactions rather than outcomes. The short-term aim should be to improve the understanding of activity costs, but over time efforts should focus more on outcomes.

Data have been 'pulled through' into decisionmaking. There have been improving data on implementing partners' activities, and examples of learning and adaptation. However, a more effective system for measuring outcomes and quality is needed for the programme to move forward. In general, feedback and learning mechanisms were weak or under-resourced, and outcome data is used only to a limited extent. needs; justifying and securing budgets; coordinating activities; delivering value for money and management for results; and monitoring and evaluation. There is no tight separation between achieving efficiency and the other DAC criteria (such as relevance and sustainability), but it is helpful to provide a separate analysis of it. In discussing each stage, it is recognised in this evaluation that stages overlap and have mutual dependencies. Ideally the data needed for analysis of each point would be complete. However, data were often not fully available.
The evaluation therefore arrives at judgements based on available data and recommends improvements in the collection of data.

This chapter is structured around the cycle of delivering efficient services in Figure 4.1. Table 4.1 above provides an overview of how the Programme has met its objectives in relation to the evaluation questions for efficiency. These ideas are explored in more detail in the following sections. 


\subsection{Identify needs and efficient delivery mechanisms}

This section addresses the first stage in Figure 4.1 , building on the discussion in Chapter 2 regarding how understanding needs contributes to the relevance of programming.

Needs assessments and surveys contribute to allocative efficiency by ensuring that the allocation of resources accurately reflects the distribution of need. Overall, through surveys and other data sources, the needs of refugees in Jordan, including educational needs, are identified at a level suitable to meet the requirements of decisionmakers. UN agencies (including UNICEF) play a leading role in identifying such needs but there are many other data sources, as laid out in the references to this report. For example, a number of humanitarian agencies, including IFRC, Oxfam and CARE have conducted needs assessments. The Programme has been supported by a number of data exercises including high-level tracking of the flow of refugees, identifying their needs at the point of arrival, and identifying education needs. Similarly, UNICEF's 2013 Report Shattered Lives contains a rich analysis of the challenges facing Syrian children in Jordan. ${ }^{84}$ Yet another example of needs assessment supporting the allocation of resources is the annual safety audit narrative, ${ }^{85}$ which makes transparent linkages between the evidence of need and the relevant response.

The use of even more granular data to shape the response can also be seen in the development of Joint Proposals (discussed below). At the time of writing, the UNESCO Amman Office, responding to a request from the $\mathrm{MOE}$, is preparing to adapt and use the UNESCO OpenEMIS (Open Source Education Management Information System) software for the Jordanian education system. Relevant data are therefore becoming more available to decisionmakers.
There are several good examples of this data being used to inform decisionmaking. For example, in 2013 UNICEF worked with the MOE on a 'school capacity mapping exercise' which led to UNICEF supporting the establishment of 98 double-shifted schools and provided 65 prefabricated classrooms to 26 schools. Some 64 schools received school furniture and another 44 received small-scale renovations. ${ }^{86}$ Another example is that by 2013, RRP6 (p.53) evidence about aggregate cost drivers was informing overall strategic thinking: it was argued that the number of 550,000 Syrian refugees in October 2013 was expected to rise to 800,000 by the end of 2014 and it was assumed that some 36 per cent of these people would be school aged. These data (however imperfect the predictions proved to be), along with assumptions about the needs of children with disabilities and pre-school children, were used to support financial planning.

How these data have been used in other cases in the EER to arrive at an evidencebased allocation of resources is less clear. This is apparent when comparing key planning documents. For example, the 3RP (2015-2016) brought together plans developed by national authorities affected by the Syrian crisis, including the Kingdom of Jordan. Within this, the Jordan Response Plan comprised 11 sector strategies of which education is one. 3RP followed the 2014 UNHCR Regional Response Plan (in its 6th version). In comparing these two planning documents it is apparent that the scale of resources needed for different activities appears to be inconsistent over time. For example, if the 3RP and RRP6 are compared, different response requirements can be seen. These are summarised in Table 4.2 overleaf.

The first issue is that two key documents supporting decisionmaking processes for the Programme at the strategic level arrive at 
Table 4.2: Summary comparison (where possible) of budgeting requirements between 3RP (2015) and RRP6 (2014, January to June only)

\begin{tabular}{|c|c|c|}
\hline & $\begin{array}{l}\text { Refugee response and } \\
\text { resilience response } \\
\text { requirements (3RP) (2015) } \\
\text { Whole year }\end{array}$ & $\begin{array}{l}\text { RRP6 (2014) January to } \\
\text { June only of findings }\end{array}$ \\
\hline $\begin{array}{l}\text { Early childhood development in vulnerable } \\
\text { communities }\end{array}$ & $\$ 5,783,750$ & $\$ 1,220,520$ \\
\hline Teacher training programme & $\$ 6,295,000$ & $\$ 1,115,081$ \\
\hline Alternative certified education pathways project: & $\$ 22,396,000$ & $\$ 12,129,727$ \\
\hline Violence-free schools & $\$ 346,600$ & \\
\hline $\begin{array}{l}\text { Equal access to education opportunities for } \\
\text { Jordanians }\end{array}$ & $\$ 41,579,096$ & $\begin{array}{l}\$ 8,970,035 \text { Jordanian public } \\
\text { schools supported with } \\
\text { additional learning spaces }\end{array}$ \\
\hline Expansion of higher education & $\$ 57,250,000$ & $\begin{array}{l}\$ 5,950,800 \text { Youth benefit } \\
\text { from Secondary and higher } \\
\text { education }\end{array}$ \\
\hline MOE enhanced institutional capacity project & $\$ 650,000$ & \\
\hline Formal education & & $\$ 12,137,292$ \\
\hline $\begin{array}{l}\text { Children with specific needs access educational and } \\
\text { psychosocial services }\end{array}$ & & $\$ 1,206,600$ \\
\hline $\begin{array}{l}\text { Essential learning materials including stationery and } \\
\text { other supplies }\end{array}$ & & $\$ 3,840,211$ \\
\hline
\end{tabular}

very different conclusions about the resources required. To some extent this is because of adding the resilience component in the 3RP. While the RRP6 estimated Jordan's 2014 total education response needs at $\$ 86,317,219$, the 3RP stated that the resilience budget requirement alone was $\$ 177,672,696$, with the refugee response requirement at $\$ 79,230,750$, making a grand total budget requirement of $\$ 256,903,446$. (Numbers from the 3RP were later revised.) Not only are differences in the grand totals hard to relate to changes in underlying needs, but also the specific resources committed to individual activities are hard to understand. The expansion of higher education to $\$ 57.25$ million in $3 R P$, for example, appears arbitrary and unrelated to the needs of the Programme (considering that only 2 per cent of Syrian refugees passed the Tawjihi exam to access higher education). Even if less money were committed to higher education, it would not clearly serve to strengthen resilience to the benefit of those targeted by the emergency response (see Annex 4 for a breakdown of the two budgets).

The second issue is that the processes for managing resource allocation are not sufficient to ensure (and demonstrate) allocative efficiency. Furthermore, decisions about how best to deliver programmes within these broad priorities do not appear to systematically follow options appraisals to identify the most efficient mode of delivery. If options appraisals were systematically conducted to review how to meet identified needs most efficiently, this is not apparent in the documentation. However, there are important exceptions: for example, the evaluation team was told how the selection of the Makani approach followed considerable discussions about how best to package activities to achieve the best value for money (although even this 
did not involve a formal options appraisal). In general, the approach adopted in the past does not appear to have been structured to select optimally designed activities.

For example, it was not apparent in formal planning documents how decisions have been made concerning resource allocation to alternative education versus the formal education system (where the large majority of Syrian children are being educated). An evaluation question is whether efforts were designed to meet learning needs assessments; however, there were not assessments that developed an analysis of options to balance needs for formal education versus alternative education. Similarly the reasons for allocating resources (or not) to teacher training/recruitment rather than to alternatives to education are not explicit in the formal planning documents, and nor did interviews clarify this. Choices appear to result in working around the GOJ rather than with and through it, creating competition between these two education approaches. Insufficient use of options appraisals, and an apparent (but understandable) preference for tried and tested humanitarian responses in creating parallel systems when a speedy response is needed, may have led to responses that were less appropriate in Jordan's context, with its own governance in place and well-developed infrastructure. Similarly, it was also reported in 2014 that Jordanian NGOs were being 'swamped by the influx of new INGOs, who often did not prioritise capacity strengthening of their local partners while imposing their model onto them'. ${ }^{87}$ It could be speculated that a response more embedded in Jordan's existing system of public infrastructure, civic entities and NGOs, and focused primarily on building that capacity, would have had better consequences in the short and medium term.

Furthermore, how the analysis of cost drivers fed into allocating resources to specific activities is not clear, including for example the MOE estimate that hosting a further 60,000 children in the public system, with infrastructure costs, would amount to $\$ 344,000,000$ (p.53) when the RRP6 earlier (p.10) states that 'for education, the annual cost of each student enrolled in the primary and basic stages is US $\$ 877$, increasing to US $\$ 1,995$ for the secondary stage. To cover an additional 80,000 students, the annual cost would be US $\$ 81.4$ million.' The range of such estimates and assumptions makes an optimal allocation of resources and selection of the most efficient delivery mechanisms difficult to achieve. For example, there is no systematic assessment of the marginal costs per child comparing expanding double shifts, creating prefabricated schools, and building new schools. Similarly, efficiency would be strengthened by understanding the drivers of cost per child which include: teacher salaries, infrastructure (buildings, furniture), textbooks and learning materials, and management.

A further gap between the needs analyses, on one hand, and the allocation of resources to specific activities, on the other, is that, in order to mitigate the effect of the crisis on the host population, the Ministry of Planning and International Cooperation (MOPIC) required projects to include at least 30 per cent of Jordanians along with targeted refugees. The beneficiaries of this were not open to negotiation by the implementing organisations. However, this evaluation recognises that there may be a trade-off between ensuring the acceptability of the programme and maximising efficiency.

As noted in Chapter 1, the EER is evolving to include not only a response to the immediate need for access to education but also a longer-term commitment to resilience and a concern with quality. This is creating the need for further data and in particular the need to monitor learning and outcomes. The Education Management Information System (EMIS) 
being developed by UNESCO (following a MOE request) to support educational data requirements may meet many of these needs.

The efficient allocation of resources within the Programme is therefore linked to the identification of needs and relevant evidencebased interventions based on analysis of various options. In the Programme, data on needs were often sufficient and credible. By December 2014, with the publication of the Jordan Response Plan 2015 for the Syria crisis, there is a clearer overview of needs and planning assumptions which are linked (however persuasively) to the refugee response and resilience response. Further improvements are still required, however, to be confident that the allocation of resources reflects the distribution of needs and evidencebased decisions among options. Additionally, there appears to be no systematic way to ensure that the programmes selected to meet these needs are the most efficient possible. These same analyses could then be used to help justify budgets to donors and secure further funding.

\subsection{Justifying budgets and securing funding}

This section discusses the second stage of Figure 4.1: justifying budgets and securing funding. The Syrian crisis continues to place a significant burden on Jordan and, as the Planning Minister Imad Fakhoury observed at a meeting with donors and international agencies in 2015 ahead of the Kuwait III donor conference, 'We must also begin to look beyond the short-term horizon'. ${ }^{88}$ Recognising the importance of strong evidence, the Minister emphasised that the formal planning process will be based on a comprehensive vulnerability and needs assessment for both refugees and host communities (raising the question of balancing a programme for Syrian refugees with responding to all unmet needs in Jordanian communities). Addressing donors and international agencies on the need for good data, the Minister said, 'we will need your strong engagement through the Sectoral Task Force mechanism'. In response, the UN official present said, 'We must engage proactively with the government in the management architecture and mobilise significant additional resources to complement already existing and generous donor contributions'. Along with many of our interviewees, both the UN and the GOJ are emphasising the need for a resource mobilisation plan supported by strong evidence. A key document for justifying and mobilising international funding in December 2014 was the 'Jordan Response Plan 2015 for the Syria Crisis'. Prior to this, the 3RP had identified further, and greater, funding needs for 2015-16. As planning tools, as well as fund-raising tools, these documents both suffered from a degree of cynicism regarding the amounts of funding requested (described by one interviewee as 'beyond the capacity of any donor') and the view that it was not prioritised (dismissed by an interviewee as 'a wish list'), and interviewees suggested that the rationale for the financial resources identified was not transparent. Addressing the issues identified in the previous section would also help allay such concerns.

\subsection{Coordinating and aligning for maximum efficiency}

The third stage in Figure 4.1 is coordination and alignment. Interviews with both UN agencies and implementing partners showed a strong awareness of the importance of coordinating and aligning activities and the contribution that this can make to the efficiency of the EER. Coordination and alignment in the EER is pursued through both formal and informal means. Formal coordination at the national level is led by the MOE and relevant government partners and involves MOPIC and UNICEF. 
At the sector level, coordination is led by the Education Sector Working Group, as discussed below. As is also outlined below, the alignment of implementing partners, and their coordination for the education response, is reinforced through PCAs with implementing partners. Meanwhile, under the terms of the JRP, all resilience and refugee-related projects must be submitted for review to MOPIC and relevant line ministries for approval. Interviewees from implementing partners also highlighted that informal coordination can be an effective and efficient modality and includes: co-developing strategies (e.g. gender); data sharing (e.g. the - not fully successful - sharing of data with UNHCR); and facilitating the development of innovations (e.g. Makani). Structures are in place to support coordination and alignment to link GOJ and UNICEF strategies with the activities of implementing partners on the ground. As the EER evolves to include more attention to resilience and to quality of education, the potential benefits of coordinating and of sharing experiences will become even more valuable. How well these structures work is described in the following sections.

\subsubsection{The Education Sector Working Group}

A central pillar of the formal coordination architecture for refugee education in Jordan is the Education Sector Working Group, originally established in 2008 with its mandate later extended to include the current Syrian refugee crisis. The Group is co-chaired by UNICEF and Save the Children, and it aims to support both the humanitarian response and development education programming in Jordan. A review of the Minutes of the ESWG and its annual plan suggests well-attended meetings, well-minuted processes, and a strategic focus on issues identified in the clearly articulated ESWG's terms of reference, ${ }^{89}$ which are:
1. 'To provide a coordination forum in which all the appropriate organisations and institutions collaborate with the aim to support the Jordanian education system in current and future emergencies.

2. To plan and implement a response strategy: applying norms and standards, developing capacity, responding to needs, monitoring and evaluation, and conducting advocacy.

3. To ensure continued access to quality education in a safe and protective environment for all vulnerable children. In doing so, the ESWG has a particular responsibility to ensure that programmes are accessible and adapted to the needs of all children.'

Despite this, interviews conducted as part of this evaluation suggest that although there was a broadly positive view of the ESWG, the success of such coordinating activities has been mixed (although both interviews and ESWG minutes show there have been more recent efforts to strengthen this). Some interviews indicated that the ESWG has in the past had difficulty involving the MOE, and even more difficulty in engaging national NGOs. Associated with this is a perception that the Government is uncomfortable with the proliferation of non-government actors taking on roles in the wider Jordanian education system. Despite their central coordinating role, it was reported that UNICEF had been excluded from some key government discussions and planning decisions that directly impact their work and the direction of the Programme. For example, UNICEF was not regularly invited to government meetings on the refugee crisis and had not been involved in discussions around the education and economic reform strategies under development at the time of data collecting. However, it should be recognised that aligning and coordinating decisions may on occasions be in tension with the accountabilities and 
sovereignty of the GOJ. From the perspective of implementing partners, a particular problem identified by interviewees was their own lack of time to engage.

In the minutes of the ESWG Zaatari Camp Education Working Group meeting of 3 March 2015 it was reported that the Chair asked members to write down their feedback on the ESWG coordination mechanism. 'Each member was asked to write two things that they liked about this coordination mechanism and chair role and two things they would like to improve for effective coordination. Results were compiled and will be shared to be worked on to improve this mechanism.' This step suggests concern about the effectiveness of the coordinating role of the Working Group, but that actions were being taken suggests these concerns were being addressed. A smaller Strategic Advisory Group with a maximum of ten education actors exists to provide strategic guidance. As late as 9 March 2015, the ESWG minutes noted, 'It was realized that most members did not know about the SAG and their responsibilities because of the turnover with in the sector members.'

There are therefore observable tensions in the system of coordination and alignment. There is also further room to clarify and coordinate the roles of different agencies involved in delivering the EER. The efficiency benefits that would come from strengthened coordination are acknowledged and understood by UNICEF in particular.

- UNICEF has had to adapt quickly to having responsibility for a strategic coordinating role, and one funding agency questioned whether UNICEF has the human resources necessary for this role of managing such a complex and evolving programme.

- Interviewees identified poor coordination between UN agencies, in particular in sharing data.
- Implementing partners reported tensions with government departments, in particular over lengthy and bureaucratic delays for project approval, hindering quick implementation. It was speculated that this reflected GOJ preference for a governmentled multi-donor fund. (The evaluation findings regarding the experiences of implementing partners more widely can be triangulated with those of research conducted by the International Council of Voluntary Agencies November 2014. ${ }^{90}$ )

- There is a perception among implementing partners that the space for both local and international NGOs has been squeezed. The future role of some implementing partners was also discussed, with one interviewee commenting: 'It will become harder and harder for NGOs, especially INGOs, to have space in the country.' This reinforces a reported belief among some implementing partners that the GOJ is uncomfortable about the growing independence of nongovernment agencies in education.

- NGOs reported difficulties with the eligibility of costs, specifically with low salary and support costs accepted; and organisations interviewed were concerned by uncertain funding on short-term contracts.

- A view from the MOE (from interviews) was that implementing partners in camps are duplicating activities.

- Information sharing and lesson learning among implementing partners was cited in interviews as a challenge to coordination. ${ }^{91}$

- The evaluation team was also told in interviews of difficulties in defining clear roles and responsibilities for the GOJ and UNICEF in schools in camps.

- At the same time one funder in an interview said that they were considering channelling resources more directly to Ministries. 
Therefore, although there is a suitable mechanism for achieving alignment and coordination that has significant strengths and secures participation (the ESWG), the process needs re-energising and its role clarified if it is to achieve its ambitious aims (as listed in its terms of reference) to:

1. Provide a platform to ensure that service delivery is driven by agreed strategic priorities

2. Develop mechanisms to eliminate duplication of service delivery

3. Conduct needs assessments and gap analyses which inform prioritisation

4. Develop sectoral plans in support of the realisation of the humanitarian priorities in the country

5. Application and adherence to standards and guidelines

6. Help secure funding

7. Strengthen advocacy

8. Support monitoring and reporting of the Working Group strategy

9. Engage with contingency planning and preparedness.

Challenges to coordination and alignment remain considerable. Challenges beyond UNICEF's direct control include the sector-wide transition from emergency to a protracted crisis which requires the coordination of a new approach, when there are differing interpretations of what this should look like; donors need to 'buy into' the strategy when there are other and growing demands on international funding; implementing partners are still developing their capacity to engage with these new challenges; and the GOJ faces financial and political constraints. These challenges limit the leadership role that UNICEF can play and undercut both efficiency and effectiveness. They also limit the ability to control costs and maximise value for money across the Programme, discussed next.

\subsection{Deliver value for money and manage for results}

The fourth stage in Figure 4.1 concerns delivering value for money. For the EER, responsibility for optimising delivery, controlling costs, ensuring financial probity, and thereby helping to deliver value for money is a shared responsibility. MOPIC and the MOE provide overall approval for projects. Improving management and delivery of programmes lies primarily with UNICEF in conjunction with the implementing partners. In particular, UNICEF provides a leadership and coordinating role in managing relationships with implementing partners. To this end, and as outlined in the previous Section for coordination more generally, UNICEF plays a leading role in helping the EER deliver value for money, working routinely with a number of NGOs and other partners. ${ }^{92}$

\section{Value for money by category of educational programme}

For formal education, the annual cost per student enrolled in primary and basic stages in 2014 was US $\$ 877$, increasing to US $\$ 1,995$ for the secondary stage. To cover an additional 80,000 students, the annual cost was said to be US\$81.4 million (RRP6 p.10). In terms of the cost per child in non-school programmes, the table below provides an overview for a range of key interventions for 2014 and 2015.

However, these numbers must be treated with care. For example, a small number of hours each week in informal education should not be compared with a full-time place in school in

92 ACTED, ADRA, AVSI, Caritas, DRC, FCA, Global Communities, ILO, IOCC, IRD, JEN, JRS, JHAS, LWF, Madrasati Initiative, Mercy Corps, MA, NICCOD, NRC, Questscope, RI, SCI, TDH-I, Taghyee, UNESCO,UNHCR, WVI, War Child UK. Participating Government Entity: MOE. 
Table 4.3: Cost per child of key alternative education activities interventions 2014-2015 (US\$) $)^{93}$

\begin{tabular}{|l|l|l|}
\hline Key intervention & Cost per child (US\$) 2014 & $\begin{array}{l}\text { Cost per child (US\$) 2015 } \\
\text { (projected) }\end{array}$ \\
\hline $\begin{array}{l}\text { Remedial education and catch-up classes } \\
\text { Non-certified/informal education }\end{array}$ & 200 & 386 \\
\hline Certified/non-formal education: & 180 & 282 \\
\hline Inclusive education and psychosocial services & 2000 & 300 \\
\hline
\end{tabular}

Table 4.4: Comparison of estimates of instructional time per programme

\begin{tabular}{|c|c|}
\hline Formal education ${ }^{94}$ & $\begin{array}{l}\text { - Grades 1-4: 770-800 hours per year for single shift schools } \\
\text { - } \quad \text { Grades 5-7: } 940-970 \text { hours } \\
\text { - } \quad \text { Grades 8-10: } 1000-1030 \text { hours }\end{array}$ \\
\hline $\begin{array}{l}\text { Non-formal } \\
\text { education (based } \\
\text { on UNICEF data } \\
\text { for a centre run by } \\
\text { Questscope) }\end{array}$ & 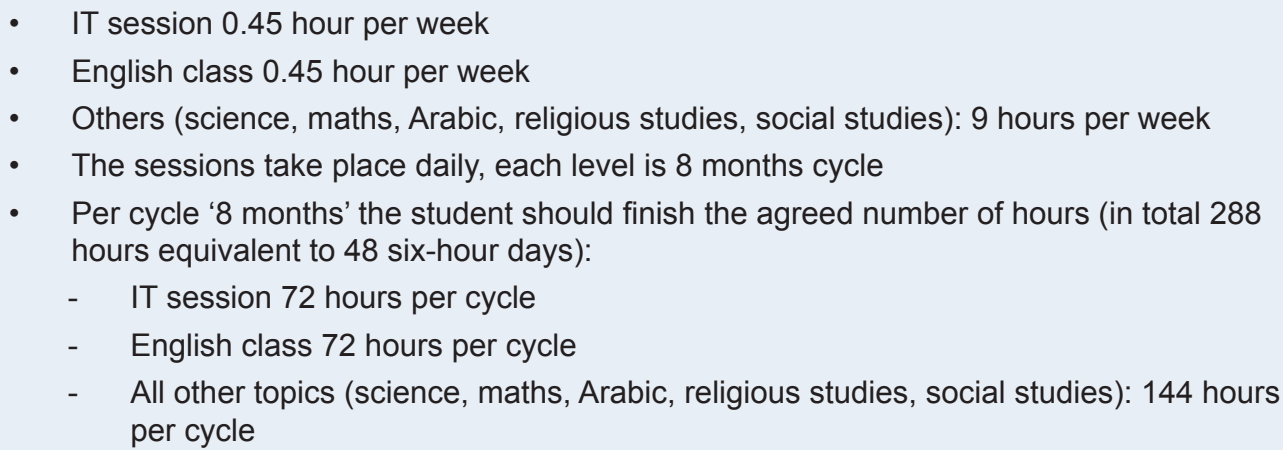 \\
\hline
\end{tabular}

terms of the experience, protection, pathways and outcomes for each child. Similarly, while the numbers of children registered in schools is known, continuing attendance (and what is being learned) is not.

Estimates are available for planned instructional time by programme, as shown in Table 4.4.

From the evaluation team's analysis of documents, including the Programme Cooperation Agreements (discussed below), it is not always possible to unpack what this might mean for children. Data on learning outcomes, or the quality of the education experience, is very limited. However, far from stakeholders being unaware of this, the issue was often raised in interviews, and in 2014 the ESWG proposed to the MOE to jointly consider how to apply the global International Network for Education in Emergencies Minimum Standards in the Jordan context. ${ }^{95}$ At the time of writing these consultations are continuing.

It is beyond the scope of this evaluation to reconstruct the data needed to estimate costs and benefits such as:

- Attendance figures

- Instructional time

93 Variations in the number of hours and the experiences offered may also explain the sudden increase in costs between 2014 and 2015 for remedial education and catch-up and for non-certified/informal education shown in the table above.

94 UNESCO-IBE (2011).

95 INEE (n.d.) 
- Amount spent on each programme for each child

- Quality of education

- Learning needs.

These data should be, but are not, readily available to decisionmakers to support value for money decisions. The limited nature of these data is a weakness in the EER that UNICEF should take the lead in rectifying. In the following section, some cost data available through Programme Cooperation Agreements is identified, but cost data are not collected in ways that support either aggregation or comparison. Comparative cost data, in particular, is important for helping to deliver improved value for money. Without it, decisionmakers do not have the important data they need to conduct a cost effectiveness analysis. The foundations for this require considerable further work, to be led by UNICEF, to more fully utilise: Programme Cooperation Agreements (PCAs), Joint Proposals, Protocols between Ministries and UNICEF, and Micro-assessments.

\section{A strategy to deliver improved value for money in the programme}

Value for money expectations are only weakly embedded in the PCAs and Joint Agreements. The relationship between UNICEF and IPs focuses heavily on transactions and not results. Comparisons of standard costs are inhibited by the way cost data is required. Microassessments also have a currently under-utilised capacity to support value for money. The ESWG addresses efficiency issues but needs a strategic planning and allocative process focused on outcomes. Options appraisals are needed and should be clearly focused on efficiency as well as effectiveness. We briefly identify below what a value for money strategy in response to these limitations would include. Drawing on a strategy already used by DFID in Jordan ${ }^{96}$ as a template, the documentary evidence supported by interviewees suggests:

1. There is no overall approach relating to $\mathrm{VfM}$ shared and understood among UNICEF, IPs, funders and the GOJ.

2. VfM expectations covering economy, efficiency and effectiveness are not properly integrated in PCAs and Joint Agreements

3. Performance targets are weakly developed within the strategic planning processes.

4. There was no formal embedding of VfM analysis in the options appraisals of new programmes.

5. Monitoring of VfM progress is weak.

6. There is little human resource within UNICEF currently to deliver this approach.

\subsection{Monitor, evaluate and learn to improve and build capacity}

Delivering efficiency is not a one-off step but a cycle of learning and improvement based on reliable and timely monitoring and evaluation that leads to identification of areas for capacity building. It helps to ensure that education activities are aligned with MOE policies and provides donors with information they need.

In Jordan monitoring and evaluation can be divided into two areas: the activities of agencies delivering education services; and the impacts on, and needs of, those requiring the services. Evaluation is the responsibility of MOE at the national level, with the ESWG playing a key role at the sectoral level. UNICEF in particular, is seen to have a lead role, and implementing partners also conduct monitoring and evaluation activities. The GOJ is committed to monitoring results against JRP targets and now requires that each approved project has a monitoring plan and reports quarterly progress. 
To achieve evaluation, educational projects must be evaluable and in turn this requires a clearly articulated Theory of Change, intended results and pre-agreed indicators. As discussed in Chapter 2, there has been no explicit Theory of Change for the EER Programme. Intended results, agreed indicators and a Theory of Change should all be articulated within the PCAs.

The evaluation team was told by a range of interviewees that monitoring and evaluation of educational programmes in 2012-2014 was weak and that quality is not always measured; more leadership is needed. This evaluation's review of PCAs confirms this. Interviewees also noted that better measures of quality are needed and that many partners operate with different indicator frameworks, and these could be unified. Strong data from monitoring and evaluation informs a shared understanding of what is working (and what is not). The evaluation team was told that in the period of this evaluation, monitoring and evaluation systems were insufficient for this task, but there have been more recent efforts to strengthen and resource them. Coordinating M\&E at this level is the responsibility of UNICEF.

More widely than M\&E, in the past feedback and learning mechanisms were weak or underresourced. However, UNICEF in particular has put more resources into this recently. Learning opportunities (often led by UNICEF) were warmly regarded by implementing partners. But despite enthusiasm for these, interviewees said that it was hard to find time, for example, to make the most of gender focal points and similar opportunities to learn and build capacity. Learning and information-sharing based on quality monitoring supports adaptation and improvement. Another (and not mutually exclusive way) would be for UNICEF to use its role more proactively to build a market of innovating providers.

\subsection{Summary and conclusions}

Efficiency is conceptualised here as a cycle, but in reality all five stages described in Figure 4.1 continue simultaneously. During the period of this evaluation, primarily focused on 2012-2014 (while considering more recent developments where feasible), the Programme struggled to establish itself as wholly efficient across the cycle of efficient delivery. Recent efforts are promising but need to be brought together in a coherent approach.

This chapter has also identified examples of positive steps towards greater efficiency. For example, in several instances, data have been 'pulled through' into decisionmaking, and data have improved about what implementing partners were doing and delivering. There is a base upon which to build, which includes good macro-level data (especially in camps), useful project level data, and a network of partners small enough to know each other. Furthermore, the ESWG provides a strong basis for further coordination, and the GOJ continues to support the Programme with national-level coordination alongside the work of UNICEF. Donors are stretched but not unsympathetic. Implementing partners collaborate well both formally and informally. Despite facing considerable pressures, the GOJ, donors, UN agencies and implementing partners all share a belief in protecting the rights of children. This is a good foundation on which to further strengthen the efficiency of the EER.

The dynamics of the crisis create continued challenges for all stakeholders as they adapt from an emergency response with priority on access to an approach based on supporting resilience with quality more firmly at its heart. That value for money and M\&E are still underdeveloped is perhaps not surprising. However, it is now important to embed efficiency and learning across the system as it evolves.

This calls for a more rigorous appraisal of options, and includes driving improvements 
through results-focused management, while strengthening routine monitoring and evaluation. The need to strengthen the use of data to maximise allocative and productive efficiency has been identified, along with the need to coordinate and align to avoid duplication, to achieve economies of scale, and to share learning. Overall this would support a more outcomes-oriented approach and a valuefor-money strategy. This would benefit from a clear analytical framework for value for money, developed by UNICEF, which could be communicated internally, to IPs and to donors.
In interviews, donors suggested that the information they were presented with was not sufficient to give them confidence that resources were being well prioritised. Visibility would be easier to achieve were the EER to move towards a more outcomes-based, and less transactions-based, approach. Further confidence, transparency and opportunities to drive improvement would be achieved by developing further monitoring and evaluation, not by increasing the demand for data on implementing partners but by sharpening the focus of monitoring onto delivering outcomes. 


\section{Findings: cross-cutting} issues

A range of cross-cutting issues interact with education, and impact access, quality and provision under the EER Programme in Jordan. Cross-cutting issues include gender and children with special needs and disabilities. Crosscutting sectors include health and nutrition, child protection (including psychosocial support) and water, sanitation, and hygiene (WASH). This chapter discusses how effectively UNICEF and its partners have met Programme objectives in relation to cross-cutting issues, broadly structured around the relevant evaluation questions posed by this evaluation. In particular, the chapter considers how well the education activities coordinate across sectors; address the gendered needs of boys and girls; provide for children with disabilities and special needs; provide a protective learning environment; coordinate with the health and nutrition and WASH sectors; and meet the principles of human rights-based approaches and UNICEF's Core Commitments for Children. ${ }^{97}$ Table 5.1 below provides a quickreference overview of the evaluation questions related to cross-cutting issues.

\subsection{Coordination and cross- sectoral cooperation}

Coordination between UNICEF and partners on cross-cutting issues takes place sectorally and cross-sectorally via the working group system. As described in Chapter 4, the ESWG, co-chaired by UNICEF and Save the Children Jordan, works through the Inter-Sector Working Group (ISWG) with the relevant sectors to ensure that cross-cutting issues such as gender are properly embedded in all activities. The ISWG also links to the country director-level inter-agency task force (IATF), set up to facilitate humanitarian action for refugees in Jordan. The establishment of this infrastructure since the outset of the emergency has greatly enhanced UNICEF and its partners' ability to respond effectively. Indeed, NGO interviewees highlighted that these structures 'encourage [partners] to work together when it comes to education'. These sectoral and intersectoral mechanisms are nested within a national strategy led by the GOJ.

Interviewees from UN agencies reported strong coordination between partners across sectors. Good operational linkages were also reported, for example, via service referral mechanisms in camps. Some interviewees mentioned that coordination between education actors and other sectors (e.g. health and protection) was stronger in the camp setting than in host communities, as co-location allowed for better outreach, awareness of needs and relationship-building between actors.

Cross-sectoral coordination has been enhanced in a number of areas by some key initiatives, which have streamlined cooperation on gender and produced impressive efficiencies in the delivery of alternative and remedial education and protection services. Close collaboration and well-established linkages between the education and protection sectors culminated in a key Programme innovation and effective practice example in late 2014: the Makani 
Table 5.1: Cross-cutting - overview of evaluation questions and findings

\begin{tabular}{|c|c|}
\hline Evaluation question & Overview of findings \\
\hline $\begin{array}{l}\text { Governance and } \\
\text { coordination address } \\
\text { cross-cutting issues }\end{array}$ & $\begin{array}{l}\text { Cross-sectoral collaboration has strengthened significantly over time thanks to } \\
\text { investment in coordination structures and key initiatives. However, increased investment } \\
\text { is needed to address gaps in operational synergies among EER partners and to expand } \\
\text { activities addressing needs related to the full range of cross-cutting issues and sectors. }\end{array}$ \\
\hline $\begin{array}{l}\text { Meeting the needs of both } \\
\text { genders }\end{array}$ & $\begin{array}{l}\text { Efforts around gender mainstreaming and capacity building as well as enhanced } \\
\text { coordination and disaggregated reporting show that gender is a Programme priority. } \\
\text { However, crucial gaps remain and programmatic focus is still overwhelmingly on } \\
\text { outreach and support for girls, undervaluing the specific and severe challenges boys are } \\
\text { facing. }\end{array}$ \\
\hline $\begin{array}{l}\text { Promoting a protective } \\
\text { learning environment and } \\
\text { integrating a strategic } \\
\text { approach to child } \\
\text { protection }\end{array}$ & $\begin{array}{l}\text { A lack of adequate psychosocial support in schools and unsafe environments mean } \\
\text { that some children face severe risks. Promising initiatives such as Makani centres show } \\
\text { a coherence of purpose between sectors and the capacity for coordinated action and } \\
\text { innovation in this area; however strategic action is needed to address gaps in MOE } \\
\text { schools in particular. }\end{array}$ \\
\hline $\begin{array}{l}\text { Coordination with health/ } \\
\text { nutrition and WASH }\end{array}$ & $\begin{array}{l}\text { Effective practice efforts to build government capacity around WASH in schools } \\
\text { and develop gender-appropriate facilities and infrastructure have had impressive } \\
\text { reach and show coherence of approach and priorities between sectors. However, } \\
\text { infrastructural problems persist in some schools, and in others, WASH facilities fall short } \\
\text { on cleanliness. Health interventions and assessments in schools have reached many } \\
\text { children. However, enhanced coordination is needed as access to healthcare remains a } \\
\text { challenge and some children go to school hungry. }\end{array}$ \\
\hline $\begin{array}{l}\text { In line with the CCC and } \\
\text { human rights-based } \\
\text { approach in programming }\end{array}$ & $\begin{array}{l}\text { While Programme efforts have been impressive, with so many out-of-school children } \\
\text { and with compromised learning environments, it is ultimately falling short of fulfilling the } \\
\text { CCC. In addition, a number of rights challenges remain related to gender and children } \\
\text { with disabilities, as well as health and nutrition, child protection and WASH. }\end{array}$ \\
\hline
\end{tabular}

centres. An example of building capacity for cross-sectoral working, they further develop the community-focused approach of child-friendly spaces and enhance the protective function of education services and spaces. Makani centres were commented on positively by many interviewees, and they have considerable face value as a promising venture. However, at the time of data collection it was too early to assess their performance.

\section{Coordination across sectors on gender in} particular has been facilitated by the appointment of gender focal points in each sectoral working group. ${ }^{98}$ Functioning as a network, the focal points support their sector to incorporate and monitor gender equality measures in programming. The approach was positively regarded by stakeholders, but there was also a concern that focal points may not have enough time to commit to this on top of their existing work-load. In terms of monitoring and evaluation, the disaggregation of reporting data by gender is already obligatory for all Programme implementing partners.

Aside from gender, according to interviewees cooperation between partners in education, WASH and health and nutrition improved over time and led to significant gains in schools and alternative learning centres. Many NGO interviewees reported close working relationships with other partners, helping them to provide for children with disabilities, address drop-outs and 
needs of both genders by mainstreaming gender concerns and implementing a range of activities addressing inequalities. Capacity has been built via gender sensitivity training with schools and implementing partners, and cross-sectoral coordination facilitated by the appointment of gender focal points. However, insecurity and scarcity associated with the refugee crisis have exacerbated the risks specific to both boys and girls. Gaps remain in gender-sensitive programming, which must be addressed to empower the boys, girls, men and women affected by the crisis and ensure that weaknesses in gender programming do not reinforce existing inequalities. The processes by which gender affects access to quality education and the disparities between Syrian boys and girls in Jordan are complex and are discussed in more detail below.

\subsubsection{Boys face education challenges particular to their gender}

Low school attendance is a major education and protection challenge for boys, and connected to a range of complex challenges particular to their gender. The evaluation team's secondary analysis of JENA survey data showed that girls in Zaatari and host communities are more likely to attend formal education than boys. NGOs also reported greater attendance at alternative pathway centres by girls than boys. The evaluation's secondary analysis of the JENA survey data showed that drop-out rates were similar for both genders in Zaatari, at 5.5 per cent for girls and 5.7 per cent for boys; however, focus groups with teachers highlighted dropout rates among Syrian boys, which reportedly increase steadily as schooling progresses.

Child labour. One in ten refugee boys and girls $(30,000)$ are estimated by the UN to be working rather than attending school, ${ }^{101}$ though 


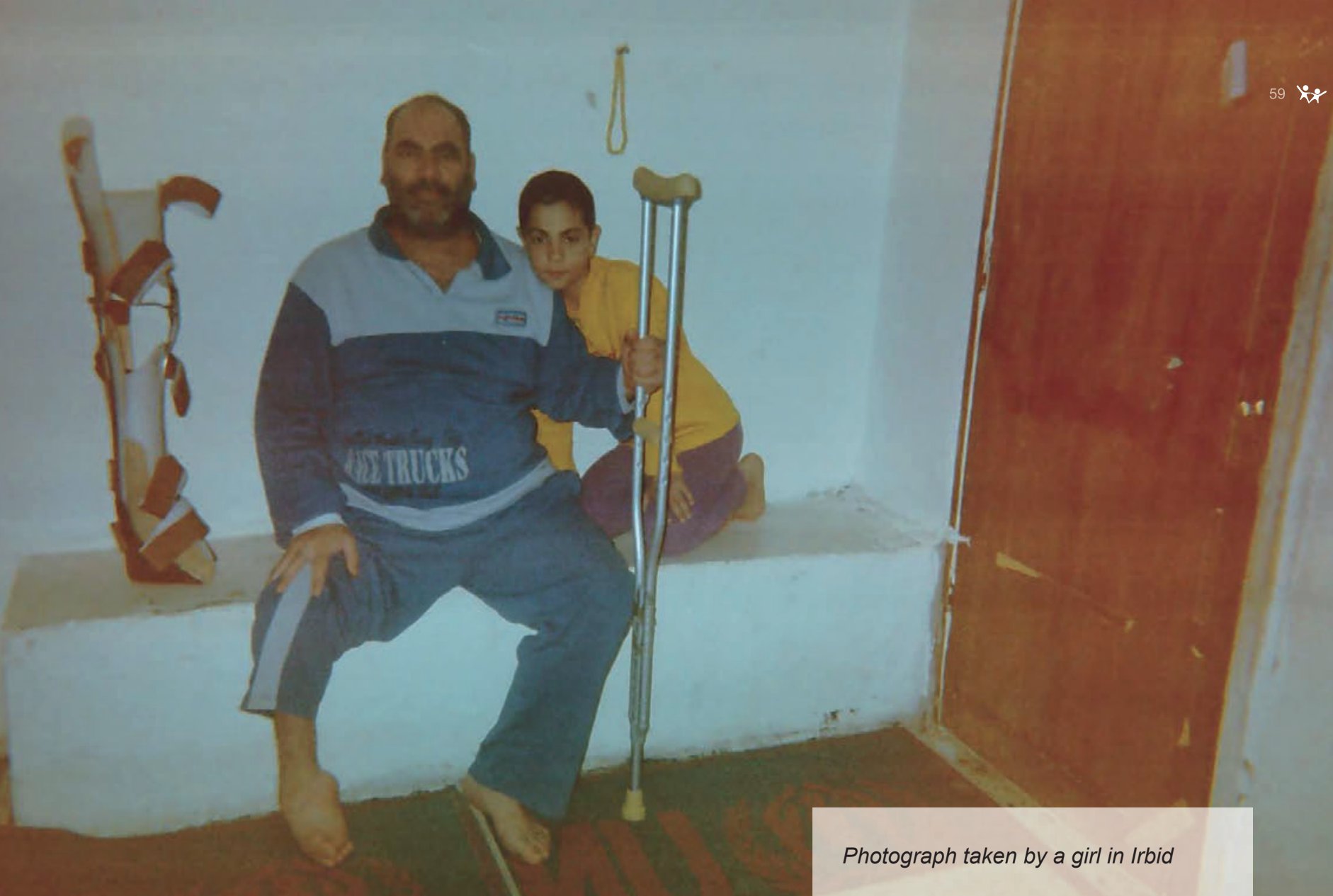

other reports put the figure at twice that. ${ }^{102}$ Regardless, the evaluation's secondary analysis of JENA survey data confirms child labour is a main reason for adolescent boys not to attend school in host communities. This is a complex issue, driven largely by households' economic need. Quite apart from exposure to dangerous, unregulated work at construction sites or elsewhere, child labourers are vulnerable to becoming separated from their families if caught by police working illegally in host communities. One UNICEF interviewee reported that at the time of data collection, 29 children with families in host communities were living in camps alone, having been sent to Zaatari by police.

Family responsibilities. In addition to child labour to supplement family earnings, focus groups with children, parents and teachers found that families rely on school-age boys to help with errands and other family duties, in particular in cases in which fathers are dead, disabled or absent. Families depend on boys to earn money and buy food and supplies, when shops are far from home, discouraging attendance in school.

\section{The conflict in Syria and the Syrian} displacement has also led to a risk of violence and abuse. A number of interviews with NGOs, UN agencies and school principals and focus groups indicated that violence in, around and on the way to school is a particular concern for boys. Unaccompanied boys in particular also face the risk of recruitment by armed groups to fight in Syria.

Lack of motivation. A major reason behind poor attendance by Syrian boys was lack of motivation, in part due to slim prospects for higher education or legitimate livelihoods in Jordan in the future, according to focus groups with children and teachers and JENA survey analysis. ${ }^{103} \mathrm{~A}$ number of interviewees also reported that boys are also often demotivated

\section{Abuqudairi (2014).}

103 Survey analysis shows that 25 children in Zaatari Camp reported that the main reason they stopped going to school in Zaatari was because they were not interested. 
and frustrated with what they perceive to be an education that does not cater to them and will not lead to future opportunities. While of course these limitations constrain girls too, this challenge was raised by interviewees in relation to boys in particular.

Behavioural problems. Focus groups with teachers found that boys had greater behavioural problems than girls, and do not perform as well as girls. Interviews with school officials in host communities supported this finding.

\section{Preference for vocational training. According} to some NGO and UN interviewees, adolescent boys often show interest in life skills and vocational training over the standard curriculum. One UN agency stakeholder noted that, given limited opportunities for progression to university, more academic schooling was perceived as less suitable to their needs. For example, in 2014 only two Syrian students were awarded a full scholarship in a Jordanian university. Interviewees from UNICEF acknowledged this gap in the provision of vocational training, but are constrained by government policy on the issue.

\subsubsection{Girls face access challenges particular to their gender}

\section{Early marriage and subsequent pregnancy} is a challenge affecting Syrian girls' access to education in both camps and host communities.

This was reported across a number of interviews and supported by the evaluation team's analysis of JENA survey data. Survey analysis from the host communities found that early marriage was the second most common reason (after lack of resources) for never having attended formal education in Jordan among girls aged 12-17. Programming targeted at girls has focused on early marriage. A 2014 study on early marriage in Jordan indicated that few girls were able to continue their education once married. ${ }^{104}$ Efforts by implementing partners to address early marriage have emphasised the engagement of community leaders to change attitudes. In Zaatari, there are a few girls married at an early age still going to school, and a few returned to finish secondary education after having children.

\section{Confinement to homes because of domestic} chores, protection concerns or social conservatism. Some girls are needed to fulfil domestic commitments at home. ${ }^{105}$ One girl in a focus group said, 'I help my mum at home because her back and hands hurt. I do the laundry, dishes and cook the food. I would like to go to school but I cannot.' An additional challenge is the limited ability of refugee women and children to leave home (for example, for education) without a male family member. Since only 20 per cent of refugees in Jordan are adult men of working age, ${ }^{106}$ and many are out of the house working, this is especially limiting. A father in Zaatari said: 'There are no appropriate recreational activities for my teenage girl, I prefer she stays at home.' Some conservative families prohibit girls from attending school after puberty, as focus group findings highlight, because of concern about interaction between males and females. Efforts by UNICEF to develop activities and technology tools for home-schooling may provide a route. However, concerted outreach to girls confined to homes, especially in host communities, should be strengthened to identify those in need and provide the full range of services and support required.

Verbal mistreatment in schools. In focus groups, some girls reported verbal and emotional abuse in schools from teachers and other students. While not reporting physical abuse like the boys, girls reported more emotional and psychological mistreatment. 
The need for gender-appropriate WASH

facilities in schools, as well as child- and adolescent-friendly spaces, was highlighted as a concern, particularly for girls. Strong progress has been made towards the Programme's plan on gender-appropriate WASH facilities, and access in camp schools was reported at 100 per cent of the target in 2014, and 78 per cent of the target in host communities. ${ }^{107}$

\subsubsection{Gender-sensitive programming and key gaps}

Gender sensitivity training in schools and with implementing partners has aimed to ensure consideration of gender in project planning and in the implementation of the EER. Cross-sectoral coordination has been facilitated by appointment of gender focal points for each Sector.

However, crucial gaps remain in the EER's gender response. Both boys and girls face severe, though different, gendered risks in Jordan, and gender-sensitive programming needs to consider both groups. Further teacher training, programming, monitoring and support is required to address children's gendered experiences. As noted earlier in this evaluation, in early 2014 the ESWG proposed to the MOE to undertake a contextualisation of the global INEE Minimum Standards for Jordan. These include standards relating to gender and should, when implemented, improve the gender response.

Although some activities are aimed at boys, implementing partner and UN interviewees confirmed that to date, gender-sensitive programming has been overwhelmingly targeted at girls. Most gendered programmes have incorrectly assumed that girls face the bulk of the gendered challenges. However issues like child labour and violence are putting both boys' access to and the quality of their education at grave risk. One example of a programme targeted towards boys, aiming at reducing child labour and increasing school attendance, was described in interviews. Two NGOs providing alternative learning pathways reported some success with an initiative distributing monthly cash transfers which were conditional on school attendance. Building on this work, greater programmatic focus is needed on the specific challenges boys are facing, alongside those of girls.

Interview responses suggest that the situation has not significantly improved since inter-agency Child Protection and Gender-based Violence assessments were carried out in camps and urban settings in 2012/2013, led by Save the Children. ${ }^{108}$ These assessments showed that some of the key protection challenges facing refugees in Jordan were lack of access to and information about services, exploitation of women and children, gender-based violence including domestic violence, sexual violence and early marriage, children separated from their families, lack of access to education and child labour. However, there have been recent efforts to address needs of boys and the issue of violence in and around schools.

\subsection{Children with special needs and disabilities}

The evaluation team's secondary analyses of JENA survey data showed that 9 per cent of children in Zaatari are reported to have disabilities, difficulties and/or chronic illnesses, while in the host communities disabilities reportedly affect 3 per cent of (school-aged) children. ${ }^{109}$ Disabilities' can be mental, physical or developmental, or

107 Wash Emergency Assistance In Jordan Schools (2014).

108 Save the Children. (2015)

109 It is not clear how comparable these numbers are, as the surveys may have used differing definitions. 
Figure 5.1: Enrolment rates of children with and without disabilities in Zaatari and host communities

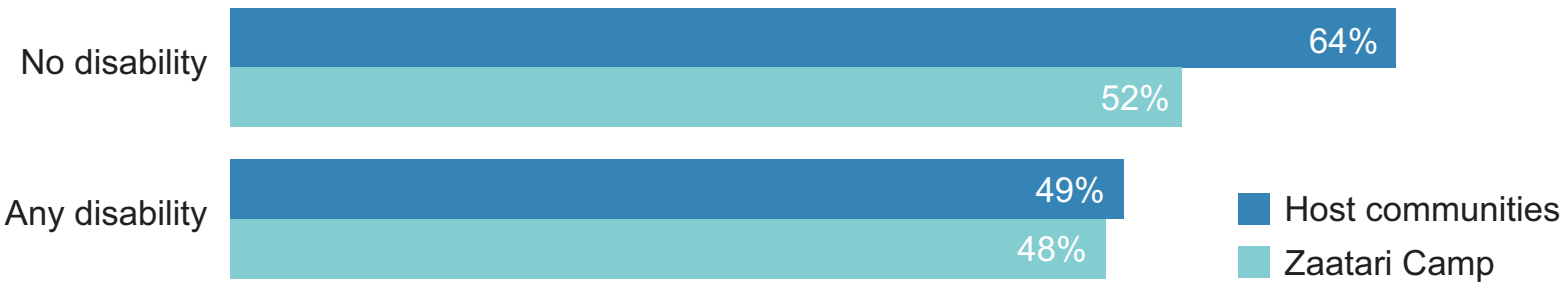

a combination. In host communities, according to RAND's secondary analysis of JENA survey data, fewer children with disabilities (49 per cent) attend school than children without disabilities (64 per cent). In Zaatari, 48 per cent of children with disabilities attend formal education compared with 52 per cent of children without disabilities (see Figure 5.1).

NGO stakeholders reported that a lack of appropriate transportation and the location of schools act as key barriers to access. In addition, parents can react to the stigma felt by keeping their children at home. In overcrowded classrooms, teachers are rarely equipped to provide the extra support a child with disabilities requires. In boys' schools, the use of corporal punishment on children with disabilities was also a point of concern for implementing partner interviewees. Children in focus groups who need mobility aids due to physical disabilities also highlighted a lack of assistance available to them, both inside and outside camps.

NGO interviewees and some teachers in focus groups highlighted that Jordanians and Syrians in MOE schools face similar challenges regarding access, social stigma and a lack of appropriate provision for special needs and disabilities.

UNICEF supports the integration of children with disabilities in formal education in camps and host communities. As of 2015, 1,983 children
(793 boys, 1,190 girls) with specific needs were provided with inclusive and psychosocial services. ${ }^{110}$ Beneficiaries were 48 per cent Syrian and 52 per cent Jordanian. The Programme's children with disabilities partner runs inclusive extra-curricular activities, teacher training, and awareness-raising activities and provides specialised equipment to schools. Success has been reported in integrating previously out-of-school children with disabilities into schools in Zaatari and elsewhere. A training manual on integrating children with disabilities into the classroom has been developed and distributed to reinforce the training delivered locally. The provision of shadow teachers to support classrooms with children with disabilities was also an effective practice described in an interview, giving teachers extra capacity and scope to manage their students' diverse needs. NGO Mercy Corps has a programme in Zaatari which has provided access to education for 1,100 children with disabilities, half of whom had no previous access to schools. ${ }^{111}$

It was reported in interviews that while there are efforts to build capacity at a school level, there is a lack of cross-government coordination on strategy and schools received little directive. While local capacity building is essential, without a coordinated cross-government strategy, gains will be limited. NGO interviewees outlined that schools receive little direction from the authorities, and in the absence of a national 
policy on the matter, at times turn children with disabilities away. While UNICEF and partners have made efforts to develop a more coordinated response between the relevant government agencies, they have not managed to build the bridges needed. As one interview participant noted, 'If they don't start working together, some categories will keep falling into cracks.'

Data collection by UNHCR on categories of children with disabilities has developed since the start of the crisis, and this information is made available to education partners providing support. However, disaggregated reporting on children with disabilities is not systematically done by implementing partners, making it very difficult to establish a clear picture of need and numbers. Most children with disabilities are identified via referrals from other implementing partners, but referral systems were reported by NGO interviewees to be limited. According to an NGO partner, no systematic referrals are made from actors working outside the Programme. Particularly in host communities, where data is even harder to access, there is danger that children with disabilities are getting lost in the system.

The long-term planning and strategic thinking needed to make sustainable improvements for children with disabilities in the Jordanian education system are also hampered by shortterm funding cycles and long delays in the issuance of government approvals for NGO programmes. With permissions to work in formal schools and activity plans only running for up to one year at a time, partners have not been able to implement sustainable programmes or build systems that will outlast international donor support.

\subsection{Health and nutrition}

Children's health, healthcare and nutrition also interact with their access to education, and in terms of service provision, collaboration between the relevant sectors offers scope for efficiencies and mutually reinforcing benefits. UNICEF and partners have built synergies between health, nutrition and education to some extent, especially in camps. However, further investment is needed to fully utilise educational spaces to build on health and nutrition education.

Interventions highlighted in interviews with UN staff include infant and young child feeding promotion and counselling; health checks, routine vaccinations and emergency immunisations at schools; and nutritional support and guidance offered to mothers. Stakeholder coordination on health in camps has allowed delivery of school-level health assessment and intervention, a version of which also takes place across the Jordanian school system. In addition in January 2015, in partnership with the World Food Programme, UNICEF launched a winter assistance programme in Azraq and Zaatari. UNICEF provides cash assistance (some $\$ 20$ for every child under the age of 14 ) via electronic voucher debit cards, with which refugee households can purchase food at supermarkets. ${ }^{112}$ Partners in host communities also reported open days which offer free healthcare to the whole community. A UN staff interview participant reported, however, that health education has been limited thus far, requiring involvement of the Ministry of the Interior to scale it up.

Focus group discussions with children and parents in Azraq camp indicated that access to health services for their children are limited. No dentistry or optometry services are available and the location of the health clinic, combined with limited transportation, means that the sick or injured need to walk challenging distances to access care. However, despite this, one UN interviewee reported that thanks to stronger coordination among stakeholders, health and nutrition were far easier to monitor and manage 


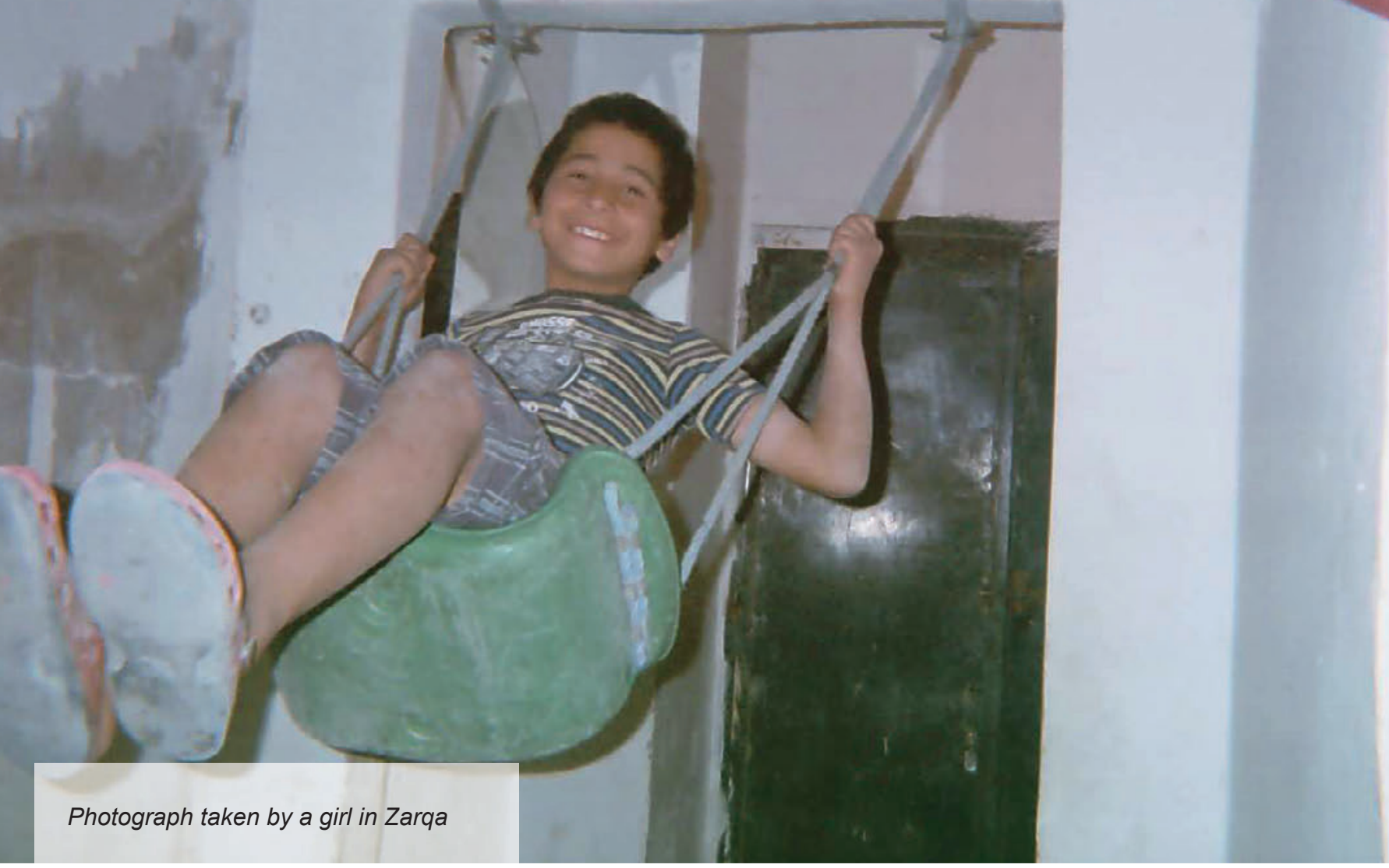

in camp schools than in the host community setting. Indeed, in focus groups one mother with a chronically ill son highlighted how access to health services in host communities had become more difficult. Due to changes in health insurance policy for Syrian refugees, medication and some specialised health services are no longer provided for free, creating a new financial burden.

Another UN staff member raised the concern that unlike in camps, sufficient calorie intake for children was difficult to ensure in host communities and children in need were hard to identify, especially given that malnourished children would often be unlikely to attend school. One UN interviewee noted that data gathered by implementing partners in Zaatari indicated a low prevalence of malnutrition, and that initiatives are ongoing to support vulnerable groups. However, focus groups indicated that children are still going to school hungry, which can affect their concentration and their educational attainment levels.
Focus groups with children and parents and interviews with UN staff indicate that children's access to healthcare, whether due to lack of transport, distance or financial pressures, is still a challenge in both camps and host communities. Where needed, transportation allowances or alternative arrangements must be provided to ensure that sick or injured children can access the services they require. Increased coordination between stakeholders is needed, especially in host communities, to develop a programme that tackles the nutritional and healthcare needs of children efficiently and effectively. School snack programmes for vulnerable Syrians and Jordanians, for example, would offer a straightforward partial solution. Finally, government engagement is needed to develop a scaled-up programme of health education in schools and to make the most of potential health and education synergies. 


\subsection{Child protection}

Child protection encompasses activities designed to protect children from violence, exploitation and abuse ${ }^{113}$ and, within the bounds of the Programme, seeks to create a protective learning environment for all children in Jordan. Protection challenges interact both directly and indirectly with education for the displaced in Jordan and include unaccompanied and separated children, child labour and associated police crackdowns, children associated with armed groups, violence threats and bullying against children, ${ }^{114}$ and higher levels of SGBV and abuse. ${ }^{115}$ Activities are managed under the Child Protection Sub Working Group, co-chaired by UNHCR and UNICEF representatives, and include capacity building with government and partners, prevention and outreach, psychosocial services and the development of referral and case management systems. Through the Sub Working Group, coherence of approach and coordination between education and protection has been strong in a number of areas. This has allowed for gaps to be identified around monitoring, case management and referrals and for some ambitious and largescale innovations which address mutual priorities, such as Makani centres.

While gains have been made in building capacity for monitoring and the identification of protection needs, referral systems to education were reported still to be lacking. The conflict in Syria and the strain of displacement has created severe psychosocial challenges for many children, and while a range of training and support services have been rolled out, focus groups and interviews suggest urgent unmet need. Many interviewees from NGOs, schools and UN agencies also reported that overcrowded classrooms are creating risks. Inexperienced teachers in camps and second shifts and overcrowded classrooms make effective classroom supervision difficult, and focus groups with children underlined the violence and bullying that takes place in and around schools. Some girls in focus groups also reported verbal mistreatment and emotional abuse not only by students in schools, but by teachers too. For boys in particular, violence is a major issue, both between Syrians and Jordanians and among Syrians themselves.

Overall, system-level improvements are required, informed by teachers, students and parents, to address the pressing psychosocial and protection needs of Syrian and Jordanian children, both within and outside schools. A coordinated and comprehensive strategy needs to be developed, facilitated by UNHCR and UNICEF, which engages the GOJ and implementing partners, in order to systematically address urgent protection gaps across MOE schools and to improve and expand provision psychosocial support.

The sections below discuss these issues in more detail, offering an overview of key protection challenges facing Syrian and Jordanian children as well as the Programme's responses.

\subsubsection{Referral linkages and identification of need}

Protection needs and gaps are monitored and measured in a number of ways. Each partner working on child protection reports bi-weekly on vulnerable children they have engaged with, allowing UNICEF to track specific children and their attendance to child-friendly spaces through the year. While one UN protection specialist interviewed reported that child protection had strong linkages with other services generally, systematic identification and referral 
mechanisms into education programmes for Syrians and Jordanians were reported to be severely lacking. With this in mind, joint meetings to improve referral mechanisms between child protection and education actors have begun, and should be a key focus of education-protection joint working efforts to improve coordination and coherence. A violence-in-schools survey supported by UNICEF and the MOE was also ongoing at the time of data collection, with expectation for future action - such as a case management system - for individualised tracking of child victims of violence.

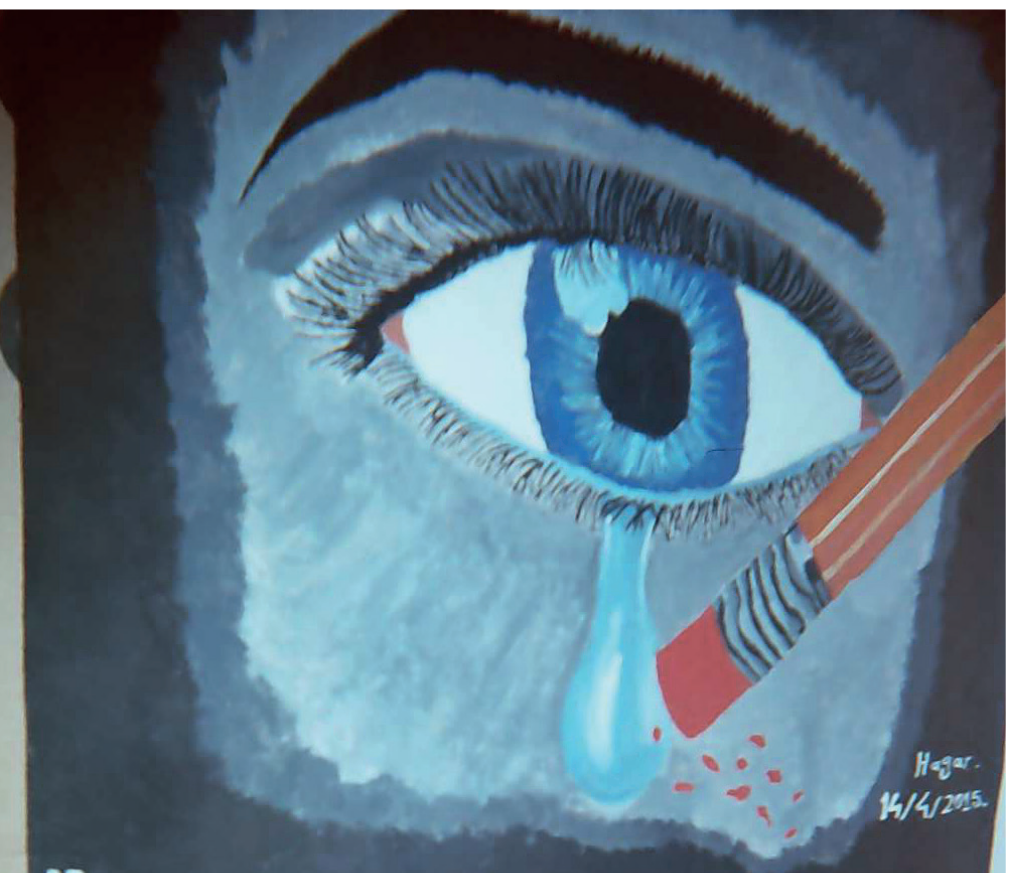

01

Students who

are loved at home.

come to school to weARN. arent. and students who to LOVED come o

116 IMC \& UNICEF (2014).

117 UNICEF (2014d).

118 UNHCR (2014b).

\subsubsection{Psychosocial support}

The psychosocial well-being of Syrian refugee children has been severely affected by the war. A report by UNICEF/International Medical Corps published in December $2014^{116}$ noted 'Syrian refugee adolescents living in the Zaatari refugee camp struggled with grief, fear, and sadness, in addition to witnessed and perceived violence within the camp.Displacement and abrupt changes in family, school, and lifestyle are reasons for adolescents having to cope with stressful environments.'

Many interviews across stakeholder groups also reported that psychological trauma among Syrian students was widespread, and at school this was often reflected in aggressive and disruptive behaviour. Parent and teacher focus group discussions affirmed that Syrian children have unique psychological, emotional and social needs. Programme partners have undertaken a range of activities to address this challenge. In 2013, around 110,000 Syrian children (of which 53 per cent were girls) were given psychosocial support through a network of child and adolescent friendly spaces and multi-activity centres, both in camps and in host communities. ${ }^{117}$ UNICEF's key figures from 2014 also note that 19,367 boys and girls benefitted from a psychosocial development course offered in formal schools, including extracurricular activities, recreation activities and sports. However, overall the number of adults and children benefiting from psychosocial support services was still under 30 per cent of the RRP6 goal in April 2014. ${ }^{118}$ Interviews with UN and NGO partners indicated that psychosocial support systems were considered more advanced in the camps than host communities, as the confined environment and UNICEF's role in formal schools allowed for better reach. The effectiveness of these programmes is unknown. 
Despite these efforts, focus groups and interviews indicated that challenges and gaps in the provision of psychosocial support remain. There is a shortage of counsellors catering to the school system, meaning that even when counsellors are assigned, they are responsible for an unmanageable number of students. The evaluation team's interviews with school management and teachers highlighted that $\mathrm{MOE}$ schools lack functional identification and referral systems or trained counsellors. Focus groups revealed that in some cases teachers provided counselling, rather than trained counsellors, and teachers and parents were often unaware of available psychosocial support. When counsellors were assigned to a school, they were often overburdened and lacked a clear job description. In interviews, several NGO providers indicated that they do not have adequate resources or supports. In general, focus groups with children highlighted that students mainly receive support from their families and friends and sometimes individual teachers. Discussions with children also revealed that some did not want to or were not able to talk about their emotions and experiences and thus may not be willing and able to ask for support.

Training and support for teachers to deal with students' psychosocial needs were also identified as a key gap. Management staff in camp and host community schools did not deem the training provided sufficient, in terms of regularity or content. Indeed, camp schools also highlighted that having come straight out of university, teachers' inexperience meant that they had particular difficulty managing protection and psychosocial support issues. Although a psychosocial support hotline for teachers has been set up by the MOE, it was not mentioned as a resource in any of the schools visited for interviews.
In terms of alternative pathways, inclusion of a psychosocial component was mandatory for UNICEF's partners working in informal education. Since 2014, many child-friendly spaces, previously the focus of the psychosocial response under child protection, have evolved into Makani centres. The expansion of this initiative offers new scope for the integration of education and psychosocial support services.

Overall, structured systems are lacking to facilitate and monitor the provision of psychosocial support services in schools in Jordan. While UNICEF's continued roll-out of education-protection innovations such as Makani centres offers promising (but unproven) opportunities for future improvement, it is clear that many children lack access to the basic support that might help them to deal with their situations and trauma. Teacher training and the provision of adequate school counselling services that are proportional to student numbers are immediate and urgent needs in formal schools. Cooperation and coordination among partners must be enhanced in order to improve the roll-out and support of services in host community schools in particular.

\subsubsection{Child safety and bullying in schools}

A recent UNICEF study found that bullying was widespread in Jordanian schools, ${ }^{119}$ and that Syrian children in Jordan are also likely to suffer. Focus group discussions and interviews conducted by the evaluation team emphasised a number of key issues related to the school environment which threaten child safety and well-being and demand urgent action. Children's descriptions of their education environments varied to a significant degree both within and across schools. Most focus groups offered both 
positive and negative stories about their schools regardless of shift, and some children talked of Jordanian friends they had made. However despite these positive variations, the severity of the bullying described by some children and the lack of a coordinated strategy to address these challenges to child safety and well-being mean that cooperation with the MOE is required in order to establish system-wide improvements.

\section{Children's sense of safety is compromised by student-to-student bullying. Physical and} verbal bullying among students is prevalent in both camps and host communities and was a strong theme in focus groups. Interviews with school management and focus groups with children reported bullying by Jordanian children for being 'different' as well as among Syrian children (across ages, genders and regional boundaries). Such incidents include physical fights breaking out among children, violent threats or children insulting each other. One child described their experience: 'The older children bother the younger ones, they bully the kids and tell them to give them all their money. Some Jordanian older kids have knives, they come from another school and wait at the door for the Syrian kids to leave the school. They bully them and take their money and run away'. One student explained how these incidents made him feel: 'When we fight I become devastated, especially when they say bad things about us.' A Syrian boy in a host community focus group explained why he thinks they behave this way: 'to break our spirit'.

Bullying was reported by children in focus groups in both host communities and camps to take place in schools, in transportation to school, and during changes between school shifts. Interviews with NGO and UN staff confirmed that journeys to schools and limited transportation are key to protection concerns, as long waits at bus stops and walks to school expose children to risks of violence and abuse. Children in focus groups revealed that they feel like outsiders in closed community settings in Jordan and some children feel unsafe walking in the streets. Some Syrian children in the focus group discussions exhibited a sense of helplessness in situations when they are bullied by their Jordanian peers. They seem unaware of their rights, with parents advising them not to fight back because they are 'visitors'. Indeed, it was observed in focus groups with parents that some parents of children attending host community schools felt helpless as they were neglected and not listened to by teachers and the school. Some boys in focus groups with Syrian children in host communities said that they avoid direct confrontation with Jordanian boys by walking in urban areas with a group for protection. Interviews with UN staff in camps also described efforts to ensure children walk to school accompanied to address protection concerns.

Teachers' support for children varies. Some of the children interviewed identified teachers that were very supportive of them, especially at the lower grade levels where they reported teachers playing with them, listening to them and treating them as family members. As one Syrian girl said in a focus group conducted in one of the camps, 'They treat us like we are their sisters.' Another student in a focus group indicated, 'There are some teachers that encourage the poor achieving students.' In interviews with camp school management, the use of Syrian assistant teachers was noted to have greatly improved student safety and decreased incidents of violence. The extra capacity they provide has allowed monitoring of students in and outside of class. According to a few teachers in focus groups, as outreach agents they have also helped to combat truancy, bring children back to school and decrease child labour.

Other students in a host community focus group with students reported physical or verbal abuse by teachers but did not view these behaviours to be a result of their background or refugee status. They indicated that all students in the class are physically punished. A few children in host community focus groups indicated that teachers discriminate against them as Syrians by not calling on them when they want to participate in the lesson discussion or by the 


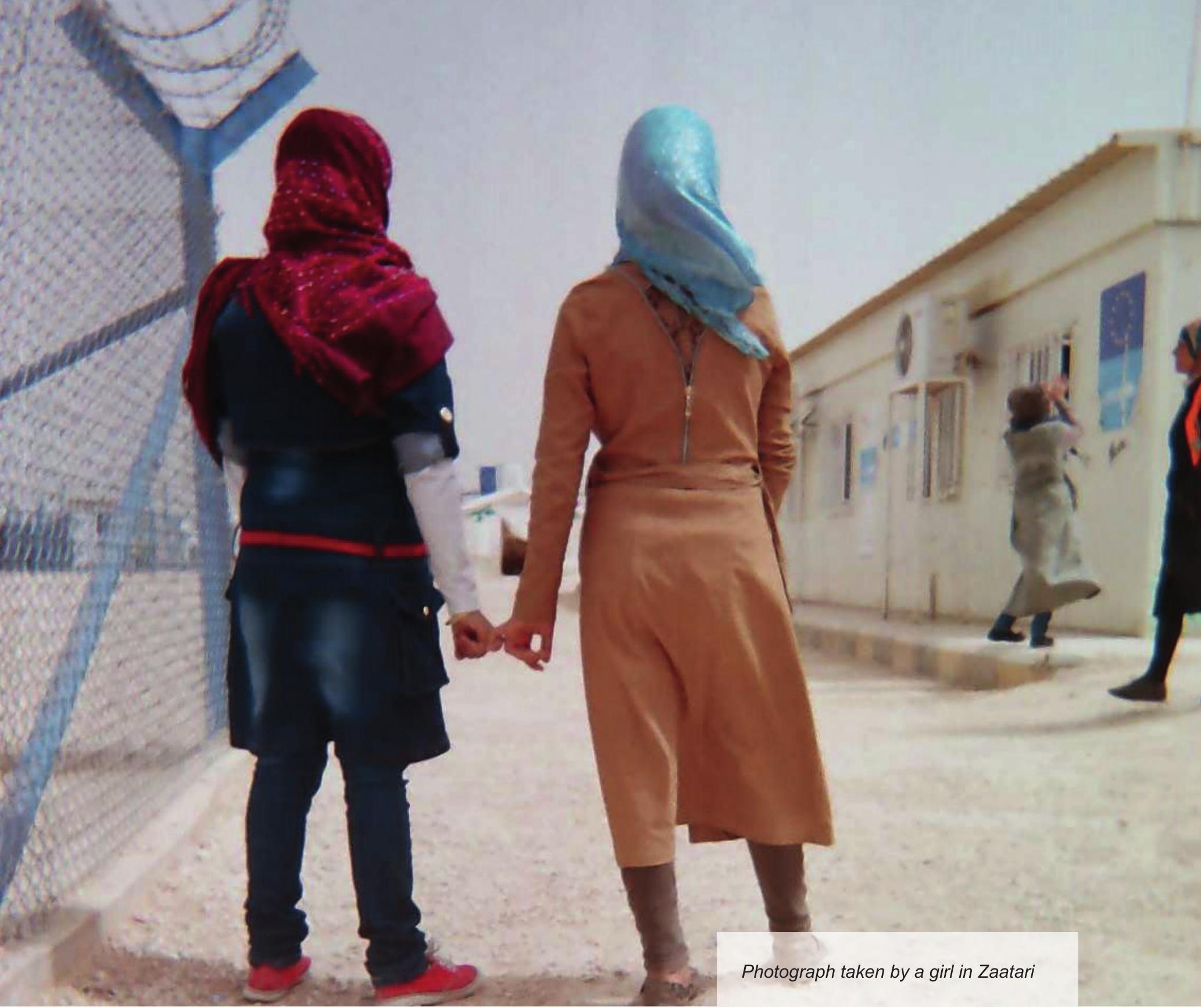

way they talk to them. The evaluation's analysis of JENA survey data from Zaatari also shows that 16 per cent of children who have dropped out of school in Zaatari reported that verbal and physical violence from teachers or other children at school was the primary reason that they stopped attending formal education. In order to change teacher attitudes towards discipline and improve school environments nationally, UNICEF and the MOE have undertaken some focused initiatives. The Ma'an campaign worked with teachers and communities against violence in schools over three years from 2009, and in 2015 the child-centred Tarbiyah Programme for School Transformation aims to build on results achieved.
While some individual principals and teachers have taken it upon themselves to work against bullying, there is no effective system in place to address the problem across MOE schools, no policies or programmes to establish a climate in which bullying is not acceptable, and no prevention or intervention strategies. A coordinated and comprehensive strategy needs to be developed in conjunction with parents, children and teachers and with the cooperation of the government and implementing partners, to systematically address these challenges across MOE schools. Improved referral mechanisms linking education and protection services, as well as the expansion of existing successful initiatives, such as Makani centres and the 
Syrian assistant teacher programme, offer promising opportunities for quick and effective impacts in this area of concern.

Table 09 in Annex 3 summarises the discussion above of the key challenges, effective practices and recommendations for future action on child protection under the Programme.

\subsection{WASH}

Coordination and coherence of approach between WASH and education has been considerable in both camps and host communities, where major investments in school WASH infrastructure have greatly improved school environments and gender sensitivity. The integration of UNICEF WASH activities in formal schools are an example of effective practice: investments stemming from the refugee crisis leave Jordan with a long-term benefit.

Capacity-building activities reported in interviews with UN and NGO staff included investments in a phased rehabilitation of essential infrastructure, the development of guidelines and standards for WASH facilities in schools and the secondment of technical advisors to the MOE. Information gathered on needs and gaps across host community schools will feed into a MOE database, allowing the government to effectively prioritise and plan future activities. Investments in infrastructure were possible due to a willingness by donors to offer long-term funding in order to avoid recurring costs. A range of interventions are also taking place in camp schools, including cleaning, waste and clean water services. At the time of reporting, gender-appropriate access to WASH facilities was reported at 100 per cent of the target in camp schools and at 78 per cent in host communities. ${ }^{120}$ UNICEF's key figures in 2014 note that 157 schools had upgraded WASH facilities, with 133,000 school children benefiting.

In terms of WASH education, sessions for students on hygiene and water conservation are also being delivered in camps and host communities. Informal education centres deliver sessions on health on hygiene and plans to comprehensively integrate WASH into programming are underway.

However, findings from focus groups and interviews show that challenges still remain in the provision of clean, adequate WASH facilities for children at home and in school. In many focus groups, children underlined that the cleanliness of toilets in schools was a problem, explaining that they try their best not to use the facilities and wait until they go home. An interviewee in a camp school also reported that water drainage problems created an unhealthy environment.

UNICEF and partners in the education and WASH sectors must continue their efforts in coordination, collaboration and capacity building to address remaining challenges and to ensure that adequate WASH facilities are available to children in schools.

\subsection{Summary and conclusions}

Human rights-based programming and gender mainstreaming are complementary and mutually reinforcing approaches that represent foundational elements in the planning and implementation of any emergency response. As discussed in Section 2.2, UNICEF's guiding Core Commitments for Children (CCC) in Humanitarian Action ${ }^{121}$ frame its efforts to protect the rights of children in all its activities, more specifically those enshrined in the Convention on the Rights of the Child. ${ }^{122}$ The CCCs related to education closely reflect the cross-cutting

120 Wash Emergency Assistance In Jordan Schools (2014).

121 UNICEF (2010).

122 Office of the High Commissioner for Human Rights (1989). 
programme priorities discussed in this Section. They commit UNICEF to: providing effective leadership for inter-sectoral coordination, equitable access to quality education for all children, safe and secure learning environments, and integrated psychosocial and health support for children and teachers. These commitments and the associated benchmarks thus provide the framework against which the evaluation assesses cross-cutting aspects of the EER.

\section{Efforts around gender mainstreaming and} capacity building, as well as enhanced coordination and disaggregated reporting, show that ensuring equitable access for boys and girls is a priority. However, crucial gaps remain and programmatic focus is still overwhelmingly on outreach and support for girls, undervaluing the specific and severe challenges boys are facing. Second, while capacity building in schools has had notable success in bringing children with disabilities into education, the systemic change needed to improve access is limited by the lack of a cross-government strategy and of coordination among actors to identify and support those in need. Third, a systematic programme of health intervention and assessment in schools, in addition to smallerscale interventions in community spaces, has reached many children. However, an expansion of services and coordination among stakeholders is needed as access to healthcare, whether due to lack of transport or finances, remains a challenge and some children go to school hungry. Fourth, in terms of child protection, a lack of adequate psychosocial support in schools and unsafe environments mean that some children face severe risks. Promising initiatives such as Makani centres show coherent planning and the potential for coordinated action in this area; however, strategic action is needed to address gaps in MOE schools in particular. Finally, effective practice efforts to build government capacity around WASH in schools and develop gender-appropriate facilities and infrastructure responded effectively to an acute identified need and have had an impressive reach. However, infrastructural problems persist in some schools, and in others WASH facilities fall short on cleanliness and hygiene, rendering them inadequate for use by students and falling short of providing school environments which are amenable to well-being and learning.

Access to education was prioritised from the outset of the refugee crisis, and in opening up formal schools to Syrian children the GOJ has demonstrated its commitment to upholding the right to education. UNICEF's commitment to a rights-based approach has been reflected in its advocacy activities, seeking to empower the Syrian community in Jordan. As mentioned in Chapter 2, this is also reflected in UNICEF's thinking around moving from 'a 'nationalitybased' to a 'vulnerability-based' approach and in its support for implementing partners' creation of inclusive education programmes and spaces for both Syrians and Jordanians. While Programme efforts described above have been significant, this evaluation lists continuing challenges and gaps which persist in each cross-cutting area and which threaten Syrian and Jordanian children's right to quality education. However, the extreme challenges of scope and the complex, difficult context faced by UNICEF and Programme partners must be taken in account, 


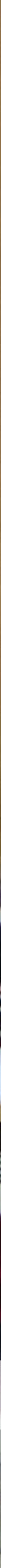




\section{Findings: sustainability}

The most widely used definition of sustainable development is 'development that meets the needs of the present without compromising the ability of future generations to meet their own needs. ${ }^{123}$ A UNESCO definition of education for sustainable development elaborates that it 'allows every human being to acquire the knowledge, skills, attitudes, and values necessary to shape a sustainable future. ${ }^{124}$ Both of these definitions emphasise that sustainability depends upon meeting both present needs and needs of the future. The DAC criteria add specificity about funding, defining it as 'measuring whether the benefits of an activity are likely to continue after donor funding has been withdrawn. ${ }^{125}$

Without an immediate end in sight to the Syria crisis and significant damage to infrastructure in Syria, it is likely that a proportion of Syrian refugees will be in Jordan for at least the next decade, and perhaps longer. If history can provide insight, the average time for refugees to return home in a protracted refugee crisis is 17 years. ${ }^{126}$ Likely assumptions are that international funding and support will not be sufficient to meet all needs; aid will decline over time; and programmes will increasingly rely on local actors (the GOJ, Jordanian civil society and the Syrians themselves).

Therefore, sustainability for the EER is the ability to provide primary and secondary education for the Syrians in Jordan for the foreseeable future, reinforced by the availability of post-secondary education and livelihood options. Based on evaluation analysis, what this means in practical terms is that:

- Appropriate education can be provided now to all the children who need it

- Provision can be scaled up rapidly to meet the needs of the out-of-school children

- Education can be provided into the medium to long term for the Syrians

- There is a plan for financing Syrian education in Jordan over time

- Quality of education will be maintained for host community children.

Based on analysis from previous chapters, Figure 6.1 shows a framework describing the factors affecting the sustainability of the EER. This chapter addresses these factors and describes how the Programme has contributed to their sustainability.

Table 6.1 overleaf provides a quick-reference overview of how the Programme has met its objectives in relation to sustainability, structured around the questions for this evaluation.

\subsection{Making trade-offs}

With budget constraints, not all goals can be met at once, and at times deeply held values can lead to conflicts in deciding which goals to prioritise. Given existing resource limitations, the need for a speedy response, and the complexity of the interacting and at times conflicting objectives and 
Figure 6.1: Factors Affecting Sustainability of the Emergency Education Response

\section{Sustainability}
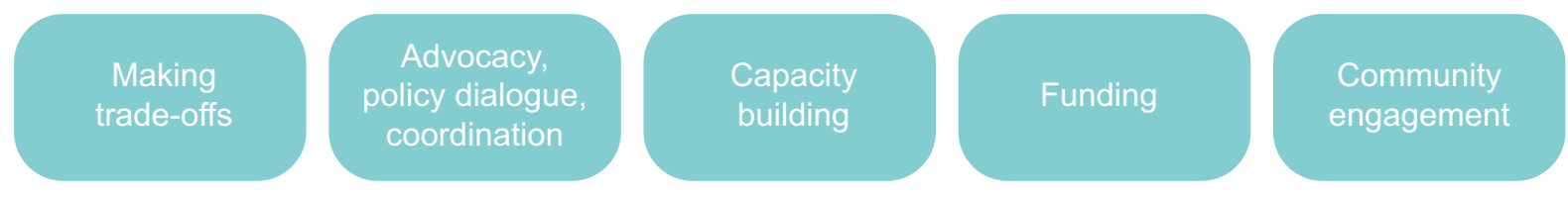

Table 6.1: Sustainability - overview of evaluation questions and findings

Evaluation question
Factors that influence
sustainability of
programme outcomes
Contribution to national
institutional capacity
building
UNICEF's efforts to
ensure that UNICEF staff
and implementing partners
had necessary capacity
and expertise

How community voices have been fed into programming and the extent to which the EER contributes to empowerment of the Syrian community

\section{Overview of findings}

Factors that are important for ensuring continuity of education into the future include: making trade-offs; advocacy, policy dialogue and coordination; capacity building; funding; and community engagement. To date trade-offs in the face of time and resource constraints have not been explicitly acknowledged and should be a key part of planning. There has been significant policy dialogue and coordination; donors and UN agencies could make better use of their influence and pool advocacy resources. Funding plans to date have been short term, and require creative longer-term thinking.

Capacity building has started, but there are further needs to build MOE management capability, sustainably pay teachers, build infrastructure and expand MOE quality assurance programmes for schools.

UNICEF responded to the refugee influx with expansion of human resources and staff skills. UNICEF have also invested in implementing partners, improving their systems and providing trainings, reflecting key priorities such as M\&E. Staff turnover and the preservation of institutional knowledge at UNICEF has been a challenge for UNICEF staff, highlighting the need for UNICEF management systems to be adaptable and agile enough to respond to the developing context in Jordan.

While the inclusion of Syrian and Jordanian community voices was not prioritised from the outset, interviews reflect stakeholders' acknowledgement of its importance going forward, for both community cohesion and appropriateness and sustainability of interventions. Existing initiatives such as the use of Syrian assistant teachers and inclusive approaches to alternative learning offer promising examples to build upon in this regard. approaches of the actors involved, an effective Education Response will have to become increasingly skilled at managing these dynamics. This will require Programme leadership to acknowledge the trade-offs that can be made in order to optimise outcomes and do the best for children in the future. Making these kinds of trade-offs, as part of analysis for the options appraisals described in Chapter 4, is important in ensuring the Programme meets its most basic objectives and uses its resources for the greatest impact. Based on stakeholder interviews and focus groups, the evaluation team noted a number of tensions in values that require making trade-offs in resources and programming.

First, given tight resources in funding, infrastructure and time, there is a trade-off between funding access and funding quality, providing wider access for more children or providing a higher-quality education response for fewer children. Access was prioritised as the 
Figure 6.2: Trade-offs affecting sustainability
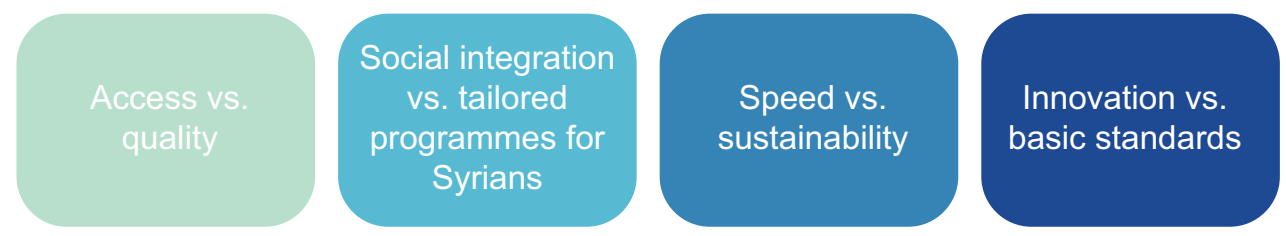

Capacity building

in Jordan vs.

international skills

first response of the Programme. Access remains a priority, and over time, prioritisation should move more toward quality. Second, there are a number of innovative programmes operated by implementing partners, including investments in technology tools for home schooling, for example. With resource constraints, the stakeholder community makes trade-offs between channelling funding towards meeting basic standards for more, or towards small innovative programmes. Third, the need to urgently meet the needs of children calls for fast responses that may be best provided by the international community; however, programmes run by the international community will be less sustainable over time and possibly more disjointed than those run by the GOJ and national NGOs, as they depend on external funding and external skills. Fourth, and related, are decisions about whether to invest in building capacity in Jordan or to draw on international skills that will leave once the crisis is over. Finally, there are trade-offs between integrating Jordanian and Syrian communities within schools to promote social cohesion over time and creating separated programmes. International literature supports the assumption that inclusion in mainstream education promotes integration and social cohesion among communities ${ }^{127}$; however, tailored programmes may better meet the specific needs of the Syrians.

Acknowledging trade-offs and making explicit decisions about them as part of options appraisals will be difficult but necessary. In making these decisions, it will be important to weigh available evidence and to rely on data on needs and performance. This creates an opportunity for a distinct advocacy role for UNICEF and underlines the need for open communication, solid monitoring and evaluation evidence and a shared, prioritised agenda among decisionmakers.

\subsection{Advocacy and policy dialogue}

In such a complex programme environment, any meaningful policy dialogue must engage all actors in order to produce relevant and sustainable results. These include UNICEF, the GOJ, donors and implementing partners. There is a significant role that UNICEF and other stakeholders play in advocacy and cross-sectoral collaboration to find solutions to challenging social and protection problems. At the same time, such communication among stakeholders has proven challenging.

One of the aspects of UNICEF's role which was considered most valuable to other agencies, partners and donors in interviews was its close relationship with multiple partners (such as the government, other UN agencies, donors and implementing partners) and the advocacy it engages in. UNICEF's ability to represent the interests of the Programme and conduct policy dialogue with the government, donors and partners has been unique and crucial to the future development of the response. UNICEF facilitates policy dialogue in an important way in its role as co-Chair of the Education Sector Working Group. 
Another key group of actors engaging at the policy level is the donors. However, while donors in Jordan have facilitated the roll-out of an impressive, large-scale response, according to interviews the group lacks coordination and a clear commitment to longer-term solutions. Neither has the donor group effectively mobilised its considerable negotiating power for common advocacy goals, and the MOE does not engage with the group as a whole. As one stakeholder expressed, donors, UN agencies and other partners need to work to develop a 'common advocacy agenda' in order to push for policy changes that may improve the protection and economic concerns of Syrians. Donors tend to negotiate bilaterally with the GOJ, leaving UNICEF and other actors in the dark about priorities and plans.

A wide range of areas of focus for advocacy were mentioned in interviews. These included the extension of non-formal education to younger children; the inclusion of Syrians in national planning; and certification for alternative learning pathways. More broadly, UNICEF's position is to advocate for vulnerable children in Jordan, regardless of nationality, which implies a continuous drive for inclusivity.

\subsection{Capacity building}

Sustaining education for Syrians in Jordan into the future depends on building the capability of the GOJ and other local actors to manage education. In addition, in order to continue to sustain and improve EER activities UNICEF must invest in the capacities and skills of its own staff and that of implementing partners. For the government, this includes: MOE management staff, school staff, school infrastructure and quality assurance programmes. For UNICEF and implementing partners, this involves ensuring staff resources are sufficient and appropriate, that skills are maintained and that recruitment is timely and strategic, according to EER needs.

UNICEF responded to the refugee crisis with an expansion of staff resources and skills. Implementing partners have also seen significant increase in human resources, to respond more effectively to the education needs of the Syrian influx. To maintain and improve the skills of implementing partners, NGO interviewees reported that UNICEF have provided training on a range of necessary skills, such as data collection and monitoring. These activities are an essential foundation to any programme development and must continue to be maintained. In addition, one NGO reported UNICEF's significant and valuable investment in their systems and operational infrastructure, which improved their capacity to respond to the crisis. However, since the inception of the EER, UNICEF itself has experienced a high staff turnover. One UNICEF interviewee indicated that this resulted in considerable disruption and led to problems maintaining institutional knowledge with regard to historical decisions around education strategy and priorities. The preservation of strategic continuity posed a challenge which, according to interviewees, affected staff ability to fully 'own' EER strategy.

While the massive increase in spending on staffing for partners and UN agencies is notable, at the MOE there is only a tiny group of people tasked to refugee issues. The MOE is also under-resourced in terms of management capacity and direct funding, while they have the responsibility of providing formal education, the bulk of the education provision for refugees. Their capacity to provide leadership, support and direction is therefore limited. If the MOE is to be a fully engaged party, it will be necessary to invest in their capacity to manage over time, for example in seconding technical advisory staff to the MOE, helping with planning, offering management training to younger MOE staff members, or providing salaries for additional refugee response staff.

In terms of staffing an expansion of the school system, government interviewees indicated that there is no shortage of people who can be hired as teachers, principals and school staff, grouped on civil service waiting lists for 
unemployed Jordanians with university degrees. However, employment depends on a consistent commitment to funding to provide adequate and fair salaries. With teachers hired for the second shifts on short-term contracts and paid between half and three quarters what teachers in first shifts are paid, their salaries of 200-330JD per month are not a living wage. In addition to being underpaid, their jobs have the additional challenges of the overwhelming classroom environments discussed elsewhere in this report. Teachers and principals in MOE schools highlighted the unsustainability of this approach.

Additional considerations in capacity building are the need for expansion of MOE school spaces and quality assurance, both discussed in detail in Chapter 3. There is need to provide additional school spaces through the creation of additional infrastructure on an urgent basis as well as in the longer term. A significant constraint to expanding provision of education and providing quality education now is a lack of school spaces. With over 500 schools double-shifted in Jordan, school infrastructure remains a major bottleneck to expanding education. An effective system to measure the quality of education of the refugees within schools was lacking during the period of the evaluation. The main quality measures currently include enrolment rates and Tawjihi test scores, which do not measure quality of education at multiple grades and in accordance with the needs particular to refugees. While the MOE has a monitoring and evaluation programme in place for its schools, it has not been able to scale up to support the additional shifts. UNICEF has programmes to measure quality in the alternative education programmes, but interviewees indicate that they are still not systematic enough. EER partners often have different indicator frameworks, which could be unified for improved efficiency and coherence. The adaptation of the INEE standards for Jordan is a promising start that has potential to enable quality measurement once implemented.
Donors can invest in expanding these quality assurance programmes.

\subsection{Funding}

Many issues surrounding funding were discussed in Chapter 4. They are raised again in this chapter because of the centrality of funding to sustainability. In particular, despite the dialogue around a shift from emergency to resilience, there has been no clear transition in approach to funding. Funding from donors for education is still based on short-term planning cycles, which was raised across the interviews as a major barrier to sustainable programming. Formal education is missing a long-term, explicit commitment from donors and an acceptance of shared burden among international donors and the GOJ. The GOJ has taken loans to cover shortfalls in government operating costs, and other Jordanian government sectors now charge the Syrians for services, such as healthcare. ${ }^{128}$ As one donor noted, 'Education is one of the last remaining systems which is providing services free of charge to the Syrian refugees.' Another donor noted, 'humanitarian resources can only go so far and are only so useful in the fifth year of the crisis. Development funds need to come on line.' What is needed is a longer-term plan for funding, with creative approaches that include but are not limited to donor funding, fully using resources in the government system, and the Jordanian tax system (if Syrians could legally work and be taxed). These issues are discussed more fully in Chapter 7.

\subsection{Community engagement and empowerment}

Sustainability also rests on the engagement of both the Syrian and Jordanian communities in the design and implementation of education programming. In interviews, a range of 
stakeholders acknowledged the importance of engaging the Syrian community. As one NGO stakeholder noted, 'The most important thing is that Syrian volunteers run all the classes. They are from the same community and culture.... We try to build on their experience. They are the system not us.'

However, interviewees also indicated that meaningful integration of community knowledge is still a major gap. One UN agency representative stated: 'We have a gap [in effectiveness] because parents have not been involved in designing the programmes.' There are few channels for Syrian voices to be heard in education strategy. Syrian adults can only teach Syrian children as volunteer assistants (not full teachers) in camp schools, and not in host communities. UN and NGO interviewees noted the weakness of both Syrian and Jordanian parent involvement with schools more broadly in Jordan, via the Parent Teacher Association (PTA) system, and the consequent difficulty of establishing meaningful engagement as part of EER activities. Overall, the sense remains that interventions under the EER are being done 'to' the Syrian community rather than 'with'. They remain beneficiaries rather than participants. Thus while the inclusion of community voices in planning and implementation of activities appears not to have been meaningfully addressed from the outset of the EER, interviews reflect the importance of community engagement and empowerment to donors, UNICEF, other UN agencies and NGOs going forward.

Alternative education activities provide good examples of inclusive approaches, with balanced attendance reported by nationality, and progress on UNICEF's advocacy of a move from a nationality- to a vulnerability-based approach to programming will be an essential element in the formation of a sustainable system. UNICEF's continued advocacy with the GOJ with regard to Syrian refugee access to employment and health services will also be key. Successful examples of engagement such as the use of Syrian assistant teachers in camps show the important role that
Syrians can play when engaged as participants in the EER, as well as the potential for building ties between Syrian and Jordanian colleagues and communities. Syrian and Jordanian communities need to be actively engaged in the Emergency Education Programme so that they can ensure that their educational needs are met according to their circumstances and so that the potential for using education services as a platform for building social cohesion between communities can be maximised.

\subsection{Summary and conclusions}

Now four years into the crisis, education provision for Syrian refugees needs to transition from an emergency response to a response that enables actors in Jordan (the GOJ, Jordanian civil society, and the Syrians themselves) to sustain education into the future. However, specific evidence of this happening is limited. In addition to setting relevant goals, implementing programmes effectively, and managing them efficiently (as discussed in previous chapters), a number of factors are important for ensuring continuity of education into the future. These include: making trade-offs; advocacy, policy dialogue and coordination; capacity building; funding; and community engagement and empowerment.

In summary, to date trade-offs in the face of time and resource constraints have not been explicitly acknowledged and should be a key part of planning. There has been significant policy dialogue and coordination, but donors and UN agencies could make better use of their influence and pool advocacy resources. Capacity building has been started, but there are further needs to build MOE management capability, sustainably pay teachers, build infrastructure and expand MOE quality assurance programmes for schools. Funding plans to date have been short term, and require creative longer-term thinking. Finally, there is room to involve the Syrians themselves more in planning and staffing the programmes for which they are recipients. 


\section{Recommendations}

The Emergency Education Response was conducted under rapidly changing, resourceconstrained and unpredictable circumstances. This evaluation has addressed the extent to which there has been a relevant, effective, efficient and sustainable programme that captures synergies and efficiencies both within the education system and from crosscutting activities. The Introduction to this report suggested that the answers to such evaluation questions would depend in part upon the yardstick used. By the standards of guaranteeing every child access to a quality education, the Programme has not been fully successful. At the same time, using the more pragmatic measure of what was delivered in the face of a massive humanitarian crisis and unprecedented pressures on the Jordanian education system, the answer is that a great deal has been achieved. The evidence presented in this report identifies significant achievements on the part of the GOJ, UNICEF and its implementing partners, donors and the international community.

Recommendations for improvements should balance suitability (focusing on resulting in the intended outcome) with feasibility (focusing on whether recommendations could be implemented in practice) with acceptability (focusing on whether key stakeholders would support the implementation of the recommendation). There is a recognition that not everything that might be suitable and feasible (for example) is also acceptable. Some of the recommendations that follow openly acknowledge that not all three dimensions are present, in particular by identifying controversies and political constraints to acceptability; but external evaluations should not recommend only what is acceptable to all stakeholders. As was acknowledged in many of the interviews conducted for this evaluation, the vision of the programme should not be constrained by what is immediately achievable. The recommendations stemming from this evaluation are presented in the sections that follow and the chapter concludes with a brief discussion of 'quick wins.'

\subsection{Build upon significant accomplishments}

Despite substantial pressures and resource constraints, the EER delivered significant achievements. The MOE, UNICEF, implementing partners and donors have enabled: structured formal education for 130,000 children, alternative educational approaches for 35,000 children, double-shifting of 98 additional schools to expand access, training for 2,100 teachers, significant resource mobilisation, and more. As children in focus group discussions confirmed, formal, non-formal and informal education has provided much needed learning, structure and purpose in their lives. There are many good examples where the Jordanian community and international organisations have mobilised to support the response, whether it be a community pooling money to buy Syrian children winter clothes or organisations providing alternative education provision for both Syrian and Jordanian children. There are many - often impressive - elements of the response that can be built on. The core features to continue to build upon include the following:

- The GOJ has made available human and physical resources, provided Syrians access to Jordanian formal schools and supported efforts to mobilise donors.

- UNICEF has provided technical expertise; coordinated with a diverse group of 
stakeholders that includes the government, donors and implementing partners; managed implementing partners; and mobilised and channelled funding.

- Implementing partners have provided expertise and implemented programmes rapidly.

- Donors have provided significant amounts of funding to enable the education response and have contributed to policy dialogue.

\subsection{Develop a clearly articulated medium-term (5 to 10-year) vision}

The Syrian crisis is not close to ending, and even when there is a peace settlement, it could be many years before there is adequate stability and infrastructure for many Syrians to return home. Jordan and the international community will be responsible for providing education for the Syrian refugees well into the future, likely for a decade or more. Despite efforts to build principals of 'resilience' into recent planning documents, there has been no real transition yet. This evaluation finds that neither key planning documents nor interviewees articulate a clear vision capable of responding to the serious challenges posed. There has been no explicit medium-term strategy developed specifically for education. Short-term programmes, with short-term funding cycles, and that lack a longer-term plan are no longer appropriate for addressing education needs. Short-term funding cycles that lead to temporary plans and breaks in programme delivery were repeatedly raised in stakeholder interviews as an impediment to sustainably addressing problems. There is now a need for concrete plans showing how services and infrastructure would be organised to deliver this vision. Interviews for this evaluation revealed that in the early years of the crisis, there was understandable reluctance from many stakeholders to plan for longer than the short term; however, there is now increasing recognition of the medium-term timescale of the crisis, likely rendering medium-term planning more 'acceptable'.
Good practice suggests a core part of this would be to develop a Theory of Change, drafted by the sector (UNICEF, ESWG, MOE) but developed nationally (MOPIC, GOJ) and shared with funders. At a 'high level' this would include: how the education emergency response as a whole should be structured in 5 to 10 years' time, and how this sits within the wider national context of education reforms to support a vibrant Jordanian economy, an inclusive society, and realistic, meaningful futures for Syrian refugees. At an operational level, the vision would include: a flexible and adaptable programme capable of accommodating an uncertain future, a small number of prioritised programmes and a strengthened monitoring capacity to learn what is working and what is not.

\subsection{Develop a medium-term outlook to funding}

Funding for refugee education is currently dependent upon resources from the Jordanian government and short-term donor commitments. It will not be enough to support mediumterm needs for education access and quality. Therefore, in support of the 10-year strategy, sustainability requires the securing of appropriate medium-term funding and in order to build resilient institutions. Funding education for Syrians in Jordan will require a phased strategy that is sustainable over time and can be adapted as circumstances change.

First, instead of short-term commitments, donors should implement a longer-term commitment cycle that enables medium-term programmes and planning - for example, a 10-year commitment instead of annual budgets. The GOJ should also grant longer-term approvals to partners implementing education programmes. This would give implementing partners the ability to provide services more efficiently, without gaps or bureaucratic delays.

Second, there should be analysis of how to secure and use most efficiently multiple types of funding from multiple bodies. In addition to 


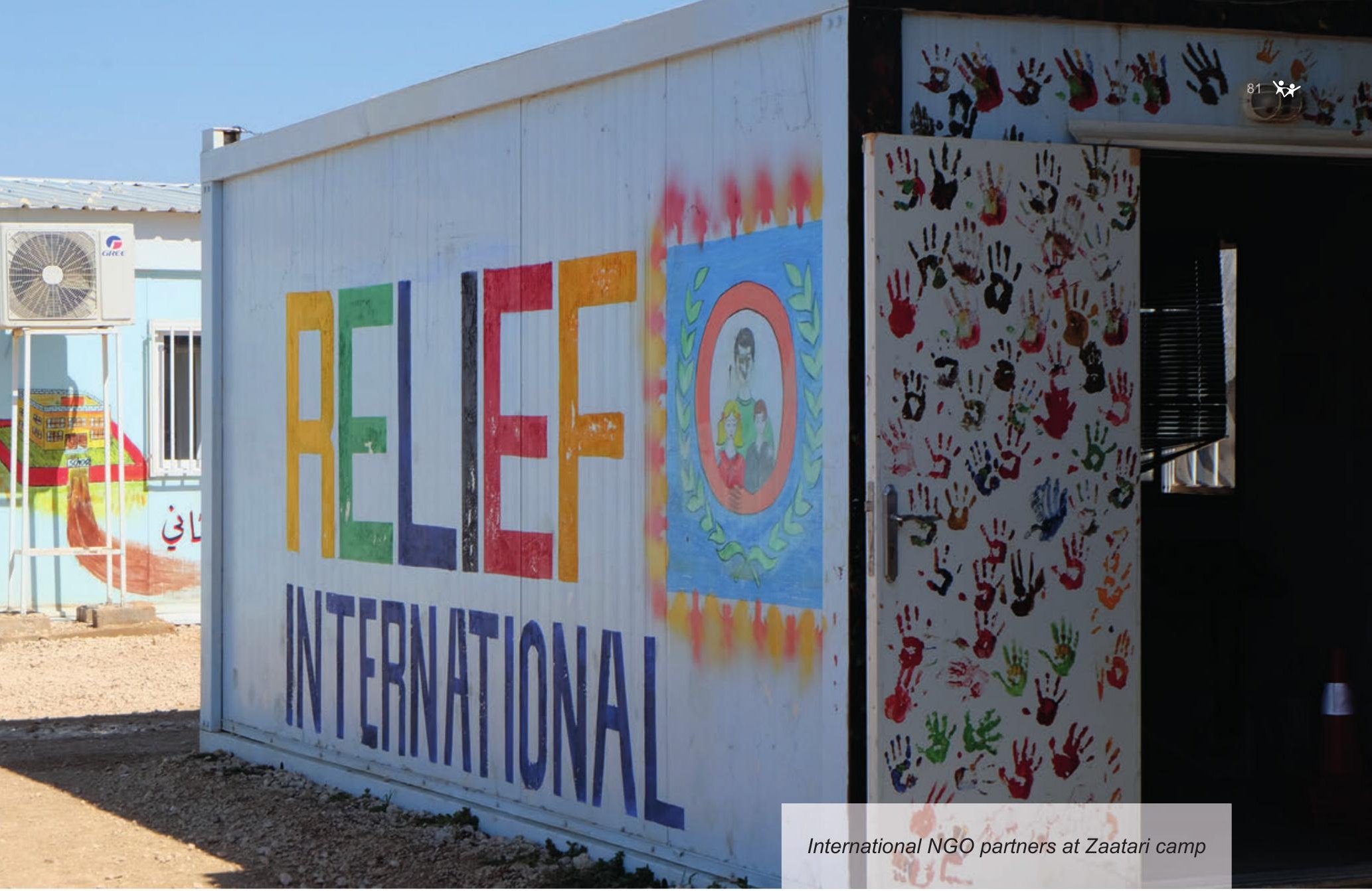

a 10-year commitment from donors and using resources in the Jordanian school system (such as MOE management, buildings and operational budgets), education could be further funded over time through other sources. One option is that the GOJ could finance education through taxation if Syrians were permitted to work in the formal employment market (Syrians are not currently permitted to work in Jordan). (This option is proposed, recognising the controversy about Syrians working in Jordan. Before pursuing this option, there would need to be further economic analysis about how to absorb Syrians in a way that is most beneficial to the Jordanian economy.) Another funding option is to use Public Private Partnerships (PPPs) to pay for school infrastructure. In this model, the private sector builds infrastructure, and the government rents it back. PPPs have been used in other countries (including Egypt, the UK and Spain) under circumstances in which governments lack the capital to build school infrastructure. Jordan's Ministry of Planning and International Coordination (MOPIC) should consider these measures.
Third, funding could be channelled to multiple bodies. Donors might consider placing more emphasis on direct support of MOE formal school operations and capacity building as the MOE will bear the bulk of responsibility for providing education over time, in addition to funding UN agencies and NGO partners. As some donors expressed concerns about accountability mechanisms within the GOJ, donors could fund the creation of such mechanisms for the activities they fund

\subsection{Prioritise expansion of formal education and creation of school spaces through investing in school infrastructure, teacher salaries and supportive planning}

Many children are not accessing the protection and skills development that full-time formal education affords. At least 97,000 Syrian children and 31,000 Jordanian children are not attending formal school. This evaluation found that there are multiple complex reasons for this, but a key 
underlying problem is the lack of school spaces. There are many children who are eligible to enter Jordanian formal classrooms who are being turned away or placed on waiting lists. According to a recent UNHCR Report, ${ }^{129}$ among families who gave reasons for their children's nonenrolment, 28 per cent cited a lack of available schools or space in a school. This is supported by interviews with MOE and UN officials, as well as focus groups with parents for this evaluation. There are simply not enough school spaces, and there is an urgent need to address this.

Substituting ad hoc alternative programming for these children with insufficient instructional hours, lack of certified pathways and nonstandardised curricula is not sufficient. There is need to develop a solid approach to education for these children with adequate instructional time (800 hours per year, per the OECD average), a certified pathway and qualityassured educational standards.

The fundamental, most central ingredients to expansion of school spaces are school infrastructure, teacher salaries and supportive planning.

To meet school infrastructure needs, there are several options. Despite the perceived difficulties raised by interviewees, each of these, and double-shifting in particular, merit further consideration:

- More double shifts: In the near term, the fastest option to expand capacity is for the MOE to open more double-shifted schools for Syrians in MOE buildings, with donors funding UNICEF and partners to manage rehabilitation of schools. Currently, only a small proportion (526 or about 15 per cent) of Jordan's 3,555 host community schools are double-shifted, and so there is significant room for rapid expansion. The GOJ should reconsider its former position of opposing creating additional double shifts to meet short-term needs. (Additional measures to improve quality in double-shifted schools are discussed in Recommendation 7.9.)

- Prefabricated schools: New prefabricated schools could be built quickly in areas with high concentrations of Syrians. This is the approach that UNICEF has taken in Turkey, building 40 prefabricated double-shifted schools for Syrian children; each school took 45 days to build (and with UNICEF 11 in just 100 days). Costs of prefabricated buildings vary, but can be significantly less expensive than permanent buildings. The GOJ should reconsider its former reluctance towards prefabricated schools.

- Renting and repurposing: Other buildings not built as schools could be rented and repurposed.

- School buses: Investment in transportation could expand efficient use of available school spaces. Some schools are not crowded, and there are many school buildings with capacity for a second shift. Taking advantage of the schools with capacity would require medium-term investment in transportation by donors.

- New infrastructure: In the longer term, donors and the GOJ should continue to invest in building quality school infrastructure. Building long-term quality schools cannot be done quickly enough to address the needs of out-of-school children or reduce the crowding that exists. This longer-term approach should be done in parallel with the short-term approach of quick expansion.

In terms of teachers, there is not a shortage of people who could be hired as teachers in Jordan if there were commitment and means to pay them for the medium-term. Jordanians on the civil service waiting list could be hired, and 
there are many Syrian teachers who could also be hired on a medium-term basis. At the salary of 400JD per month, 1,000 teachers could be hired for $\$ 6.8$ million per year plus administration costs. At the salary of 200JD per month being used currently to hire Jordanian teachers for the Syrians, 2,000 teachers could be hired per year for $\$ 6.8$ million plus administration costs. Donors should provide a 10 -year direct funding commitment for teacher salaries to scale up formal education provision, create manageable class sizes necessary for quality education, and provide a living wage to the teachers who will teach the students over the next decade.

Finally, supportive planning would contribute to formal education expansion. Ongoing initiatives to develop a database that maps capacity across school districts should be able to provide the data needed to target school infrastructure expansion. UNICEF and its partners should continue to play a crucial role in outreach and enrolment, as well as providing catch-up and remedial support.

From the evidence and discussions supporting this evaluation, it is clear that although this recommendation is suitable as a means to meet children's rights to education, and operationally feasible, it is currently not acceptable to all stakeholders and would require investments in both advocacy and fundraising for it to succeed.

\subsection{Create formal education at the scale needed for Syrian and Jordanian children who have missed years of school}

A large number (an estimated 65,000 , as discussed in Chapter 3) of Syrian out-of-school children do not qualify for formal education under MOE current policies, as they have been out of school for longer than three years. Furthermore, there are an additional 31,000 out-of-school Jordanian children. It is not feasible or desirable to try to integrate these tens of thousands of children into traditional Jordanian classrooms, when they would much older than most of the other children. Placing older children in younger classrooms is demotivating for the older children and disruptive to the grade-appropriate children. A new education approach needs to be developed for these older children.

Something akin to the certified formal MOE curriculum, offered and adapted for different age groups (e.g. starting the first grade curriculum for a cohort of 9 and 10 year olds, or offering the fourth grade curriculum for 12 and 13 year olds) should be developed and approved by the MOE and UNICEF. There are enough such children that special classrooms could be set up for them. The infrastructure needs for these classrooms are similar to those discussed in Recommendation 7.4 , with the possibility of using second shifts in current school buildings, newly constructed prefabricated buildings, or repurposed rented buildings. This should be done on an urgent basis, during the 2015-2016 school year. UNICEF and its partners can be responsible for outreach and enrolment.

\subsection{Create a new delivery model to support short-term rapid expansion of education while building long-term sustainable options for out-of-school children}

The current formal education system, by solely relying on scaling up existing approaches, cannot quickly accommodate 97,000 additional Syrian children (and in addition the 31,000 out-of-school Jordanian children). A new management and delivery model is needed. Some approaches that were appropriate in 2012 are no longer appropriate in 2015. The current management capacity of the MOE and UN agencies for the education response is stretched to the limit, and the GOJ and UNICEF should consider the best delivery mode to meet the additional mandates faced.

The first option is to rely fully on the MOE system to expand educational provision for out-of-school 
Therefore the third, and recommended, option is a hybrid model in which UNICEF would take a significant management role in the beginning, with a phased plan to transition to Jordanian MOE management over the next five years. In this model, UNICEF would oversee the creation of new certified programmes, in line with the MOE formal curriculum, for children who have missed years of school, in partnership with the MOE and NGOs. UNICEF, the MOE and NGO partners would implement the programmes in the beginning, and management and implementation would fully transition to MOE in phases. Advantages of this approach are that it could develop tailored programmes quickly that meet the needs of Syrians in adapting to a new school system, while also building capacity for Jordan, and that it would be more sustainable as MOE takes on more management over time. Education programmes could help Syrian children adapt to the Jordanian curriculum, develop participatory structures for Syrian parents and teachers that facilitate community engagement, and meet the psychosocial and other particular needs of a refugee population. Associated risks are that coordination of policies would be challenging, and phasing management responsibilities and accountability would be complex. The hybrid model provides a viable compromise and balances between the urgent need to scale up education and a sustainable approach by which the GOJ would manage the programme in the long term.

Alternative education would provide additional benefits in creating this bridge, if it were developed more strategically. Alternative programmes have the advantage of being able to be set up quickly and to be tailored to needs of the Syrian children. Some of the classrooms already set up through the alternative programmes could transition to offer the formal curriculum, tailored for the needs of the Syrians. However, to be a more effective bridge, there need to be planned pathways between alternative education and formal education.

\subsection{Invest in MOE capacity and provide direct operational support to the MOE}

The MOE currently has only a few staff who bear huge responsibility for the massive provision of education for the Syrian refugees. Resources are constrained, with the GOJ taking loans to cover refugee education and health costs. The MOE will need the human capacity to plan, manage, coordinate and lead education. International investment in human capacity for managing the crisis has been disproportionately oriented towards salaries and skills for UN agencies and NGO partners, not for the government. Regardless of the management model chosen to scale up education provision, it remains important to enable the MOE to provide quality formal education in the coming decade. The MOE already has a foundation of capacities in place, with a robust and expanding education system. However, sustainability requires avoiding the risk that knowledge, skills and resources remain in the international community, leaving Jordanian institutions either untouched or even worse off. Sustainability involves finding ways to share intelligence and data, provide joint training and support, strengthen Jordanian infrastructure, work more closely with national organisations and civic bodies, and strengthen cohesion across Syrian and Jordanian communities. This may involve trading off between immediacy of implementation, on the one hand, and long-term institution strengthening on the other.

Based on stakeholder interviews as well as good practice in capacity building in other situations, ways of developing MOE capacity (while relying on donor funding, UNICEF and external technical advice, and GOJ leadership) include:

- Seconding international technical staff or paying salaries of additional Jordanian technical staff for the MOE.

- Developing and ensuring the use of data systems. The Education Management 
Information System ${ }^{130}$ under development is evidence of further progress.

- Improving financial accountability systems. Stakeholder interviews identified accountability as a barrier to donor confidence about government capacity to manage bilateral transfers.

- Strengthening implementation and resource planning and improving communications strategies.

- Developing school monitoring programmes. While the MOE has school monitoring for first shifts, there is a need to expand capacity and develop explicit guidance circumstances involving refugees.

\subsection{Improve the performance of double-shifted schools to meet the specific needs of both Jordanians and Syrians}

In addition to the 20 double-shifted schools in refugee camps, 526 schools in Jordan are already double-shifted in order to meet the needs of Jordanians and Syrians. This evaluation recommends creating more double-shifted schools to expand access rapidly.

There are significant and continuing debates in Jordan among refugee education stakeholders about double shifts. On the one hand, double shifts have enabled an expansion of the number of school spaces, reduced crowding, responded to the concerns of parents of Jordanians who have objected to the disruptive addition of children not familiar with the Jordanian curriculum in classrooms, and have been described in focus groups as preferable by some Syrian parents, who view separate classes with other Syrians as better suited to their overwhelmed children's needs. On the other hand, the double shifts have been criticised by UN officials and donors as not offering the same quality as the single-shift schools and for setting a precedent of separate education for two communities, potentially creating a longerterm situation of a segregated society. At issue here are both the quality of double shifts and the separation of the two communities.

There are ways to use both single and double shift approaches effectively, shaped to the particular needs of the Jordanian host community and the Syrian refugees. Based on the analysis of this evaluation, there are circumstances in which all approaches are appropriate: integration, separation, single shifts and double shifts. In particular, there are circumstances in which double-shifting and separate education provide a short- or mediumterm route to managing a difficult situation and can be used to promote quality, well-being, coping and fairness. Below are recommended guidelines for using double shifts.

\section{When and how to use double shifts instead of single shifts: In cases in which schools} have become overcrowded with a fast intake of additional children, the unfortunate choice is between a crowded single shift and two less-crowded double shifts. As described in Chapter 3, double-shifting has been an effective approach that expands capacity and maintains quality in other countries when (1) resources and teachers are the same between shifts, and (2) instructional time is maintained, for example, by increasing the number of school days to accommodate shortened days. Double shifts are perceived to be a cause of poor quality in Jordan. However, the problems unique to the double shifts in Jordan are solvable, in particular if double-shifting schools offers an immediate way of expanding access for out-ofschool children and reducing school crowding. Based on findings from stakeholder interviews, school visits and interviews, and good practice 
elsewhere, recommendations for keeping equivalent quality in the shifts include (with the primary stakeholder in parenthesis):

- Add days to the school year to accommodate for shortened instructional time, to reach the OECD average of 800 hours per year of instructional time (MOE). In double shifts, instructional time was decreased from 45 minutes per lesson to 30 minutes per lesson.

- Mix up experienced and less experienced teachers in both shifts, rather than placing all newly hired teachers in second shifts as is the current practice (MOE).

- Invest in MOE school monitoring to better support double shifts (MOE, with donor funding and UNICEF technical advising).

- Ensure that the libraries, laboratories, special needs rooms and other facilities are available during both shifts (MOE, with donor funding).

- Mix Jordanian and Syrian children in both shifts (except under conditions described below), rather than educating the Jordanians in the morning and Syrians in the afternoon (MOE).

- Keep class sizes at manageable levels so that teachers are not overwhelmed (MOE, with donor funding to expand school spaces and pay teacher salaries).

- Provide adult supervision and security in the changing of the shifts to reduce bullying (MOE, with UNICEF technical advising to develop policies and training materials).

- Pay equivalent teacher salaries to teachers in both shifts (MOE with donor funding).

\section{When and how to educate the refugees} separately from the host community: When students from both communities are at the same educational level and have equivalent needs, educating them separately might contribute to a lack of social cohesion in communities. One UN official described the risk of creating 'separate but unequal' educational opportunities. However, there are circumstances under which separate education in double shifts could benefit both communities:

- When this is a short-term solution because of the crisis, with longer-term plans in place for integrated schools. Implementing wider use of separated double shifts would need to be accompanied by a plan to transition to integrated classrooms and increased infrastructure. Short-term conditions for when separate classrooms for Syrians are appropriate include when:

- Syrians have particular needs for remedial support when transitioning to a new curriculum.

- Syrians have greater needs for psychosocial support from specially trained teachers.

- In the medium term, when the Syrian children have been out of school for a year or more, and integration would imply placing them in a classroom that is not age appropriate, disruptive for both the Syrians and the Jordanians. It fundamentally weakens a rights-based approach if the rights of vulnerable Jordanians to not have crowded classrooms, with age-inappropriate classmates, are viewed as being more important than the rights of vulnerable Syrians. (As discussed in Recommendation 7.5 , a separate approach should be designed for children who have missed years of school, with formal education offered to ageclusters.)

\subsection{Improve the quality and safety of the learning environment in MoE formal schools in camps and host communities}

The focus group discussions and interviews, documentary reviews for this evaluation, as well as the results of previous evaluations all highlight significant problems with quality and safety in education: the classroom environment; low outcomes on the Jordanian Tawjihi test; 
teachers struggling with difficult classroom environments; short instructional time in double shifts; lack of pathways to further education and adulthood; bullying; and lack of systematic psychosocial support.

Understandably, quality of education has taken lower priority than access to education during the early stages of the emergency. However, the moment is now right to refocus energy around improving the quality of the learning experience for both Syrian and affected Jordanian children. Improving the quality of education is now an agreed priority. It is important that this is associated with a defined implementation, resource and communication plan. This requires a shared response from all stakeholders, with leadership from UNICEF and MOE and medium-term funding commitments from donors. Potential routes to quality and safety improvement, in addition to those listed above, are described below.

First, the MOE, donors and UNICEF should take steps to improve quality of teaching. The following steps are listed in order of priority (not all may be fully feasible given limited resources), with rationale for prioritisation discussed:

- Reducing class sizes. There is an assumption among stakeholders that teacher quality should be addressed primarily with training. However, no matter how much training is provided and practice monitored, teachers in Jordan struggle when they manage classrooms with up to 60 children, a situation exacerbated by the challenges that refugee children present. In the face of infrastructure shortages, the largest class sizes in the most crowded schools could be reduced by the MOE with the creation of additional double shifts and the hiring of additional Jordanian or Syrian teachers, funded by donors.

- School monitoring and mixing experienced and inexperienced teachers. Secondshift teachers are reported to be new and inexperienced. Expanded school monitoring, mixing experienced and inexperienced teachers, or the creation of teacher communities of practice could improve quality. This step could require few resources.

- Paying teachers adequately. Second-shift teachers receive less pay than single shift teachers. Teachers will be demotivated and have high turnover without adequate payment. At the same time, the MOE has not had trouble finding university graduates willing to be hired for the reduced salary of 200JD per month (instead of 400JD per month). Furthermore, given that many Syrians have no means of livelihood, even the reduced salary may be very welcome.

- Training. Teachers and service providers need more training on how to teach in large classrooms and deal with student behavioural problems and psychosocial issues. This training needs to be ongoing, and teachers would need to be observed and provided with feedback to ensure their success in the classroom. At the same time, training here is listed as the last priority. Training is an expensive item in the 3RP budget ( $\$ 6$ million per year, in comparison with the estimated $\$ 6.8$ million per year to pay $1,000-2,000$ annual teacher salaries). Furthermore, teachers hired are university graduates, and any training provided to them for the refugee crisis would be minimal in comparison to their university degrees; teachers in focus groups reported training already given as inadequate. This evaluation recommends that having enough teachers in manageable classroom conditions is a greater priority than a minimal amount of training.

Second, the MOE should permit better use of Syrian teachers, as they are an underutilised resource; UNICEF and donors should make this an advocacy priority. Syrian teachers have experience with a similar curriculum, and understand the circumstances that the Syrian children have come from; focus groups with parents and children found an important positive role that the Syrian teachers played with the 

address in a systematic manner the bullying in camps and host communities, which has emerged as a significant issue in focus groups. Currently, schools deal with bullying by relying on initiatives led by individual principals and teachers, as opposed to implementing policies and programmes to change school culture, perceptions and behaviours. A programme addressing all teachers and students in the MOE system, Jordanians, Syrians and others is needed. A 'quick win' could be if the MOE gave guidance to schools to have teachers monitor school grounds before school, between shifts and as the school day finishes - times identified in the focus groups for prevalent bullying. For example, UNICEF and its partners could develop programmatic guidelines and a training video, and the MOE could distribute these in schools.

Sixth, parents' voices should be engaged with in formal schools, especially in camps. PTAs are weak in host communities, and a feedback loop needs to be established in camps for parents to engage and help make improvements. The EER is generally poor at increasing accountability and participation from the Syrians themselves. Such an approach could also help to address parental attitude and student motivation, cited as key problem in Zaatari's boys' school. Improving communication between parents and schools was identified as a priority in the Zaatari JENA report, and in the host communities JENA report communication with parents was identified as a focal issue for access to formal and non-formal education.

Finally, classroom conditions, including the physical environment, furniture and electricity, would benefit from investment by donors, overseen by the MOE and UN agencies. Such investment would leave Jordan with a benefit, in addition to the costs that it accrues.

\subsection{Improve access and quality by addressing social challenges and targeting the different needs of girls and boys}

This evaluation found that access is linked to multiple complex factors involving provision of education, the location of the child and the characteristics of the family. Improving access requires addressing some of the social challenges that Syrian children and families are facing. A key issue is adults not being able to work, worsening family poverty, and making families reliant on child labour by boys and girls, and resorting to early marriage for their girls. These wider societal issues are too big to be resolved by education stakeholders alone, but the latter (UNICEF and MOE in particular) are especially well placed to sustain and increase existing advocacy from an education perspective. This evaluation can support such efforts.

Girls and boys face distinct gendered challenges that require targeted responses, both in the classroom and outside. While schools and implementing partners have received gender sensitivity training, and cross-sectoral coordination is facilitated by gender focus points, there is still a need to address gaps in gender-sensitive programming. Existing approaches have been limited. The ESWG, led by the gender focal points, should develop a re-energised approach to gender. This should include the wider societal determinants that shape educational experience and achievement. Specific recommendations, with distinct approaches for both boys and girls, should be mobilised through the wider education stakeholders.

\section{Develop approaches that enable boys to attend school and remain engaged. Boys have lower access to formal education at all levels, and are more likely than girls to become detached from the education system. This may be because of factors outside of the education system such as greater expectations on boys to work to support families, bullying, not}


seeing education as a bridge to adulthood, or because boys have greater problems adapting. Furthermore, teachers in focus groups perceive that boys have greater difficulties in the classroom, with lower engagement and more behavioural problems. This evaluation saw few programmes targeted specifically to boys, with most gender programming reserved for girls.

\section{Continue and expand programmes targeted}

at girls. Girls face a number of challenges that affect access to education. They experience high levels of early marriage, often with much older men, and pregnancy disrupts their education. Families' concerns about safety limits girls' transportation and mobility, and girls can face sexual and gender-based violence. Schools need to maintain an emphasis on genderappropriate WASH facilities and supplies. Programmes for married girls and young mothers to return to education need to be extended. Work with communities regarding girls' education is a promising practice.

\subsection{Prioritise programmes and spending based on options analyses}

Education planning has been hampered by not being informed by an explicit, detailed analysis of costs, benefits, priorities and outcomes. There is no systematic approach to options appraisals which would allow comparisons to be drawn and more informed choices made. Cost estimates have varied and are seen by some stakeholders as unconvincing. Long and costly lists of programmes that lack a medium-term vision, supporting priorities and implementation plans have been described as 'wish lists'. Resources are too limited to cover all of the desired programmes. There is a lack of data, especially on standardised costs, which weakens efforts to prioritise. Therefore, UNICEF, the MOE and ESWG should develop a formal system of priorities and options appraisals based on cost and outcome data.
There are several policy areas that would particularly benefit from options appraisals:

- Marginal cost versus average cost per child of providing formal education. UNICEF, the MOE and donors should work together to develop a cost estimate for the marginal cost of providing an additional school space, rather than the average cost of educating Jordanian children overall - for example, creating a cost estimate for using double shifts, additional costs for electricity and maintenance, different target class sizes and teacher salaries.

- Infrastructure costs for expanding school provision. UNICEF, the MOE and donors should analyse the least expensive options to expand school spaces quickly: double shifts, repurposed rented space, prefabricated buildings and building new schools.

- $\quad$ Alternative versus formal education. With limited budgets, the MOE, UNICEF and donors should assess the balance between spending to support alternative education programmes or directly funding expansion of formal education by paying for MOE infrastructure and teacher salaries. While alternative approaches are needed as a bridge, unless re-strategised they also run the risk of creating an expensive set of high-quality programmes that serve few children and do not lead to full-time certified education, at the expense of funding expanding formal education.

- Teacher salaries versus teacher training. While teacher training was universally reported as an important need, the 3RP budgeted amounts for teacher training could cover the cost of salaries for 1,0002,000 teachers, which would expand access, reduce crowding and create more manageable classroom environments for both teachers and students. 


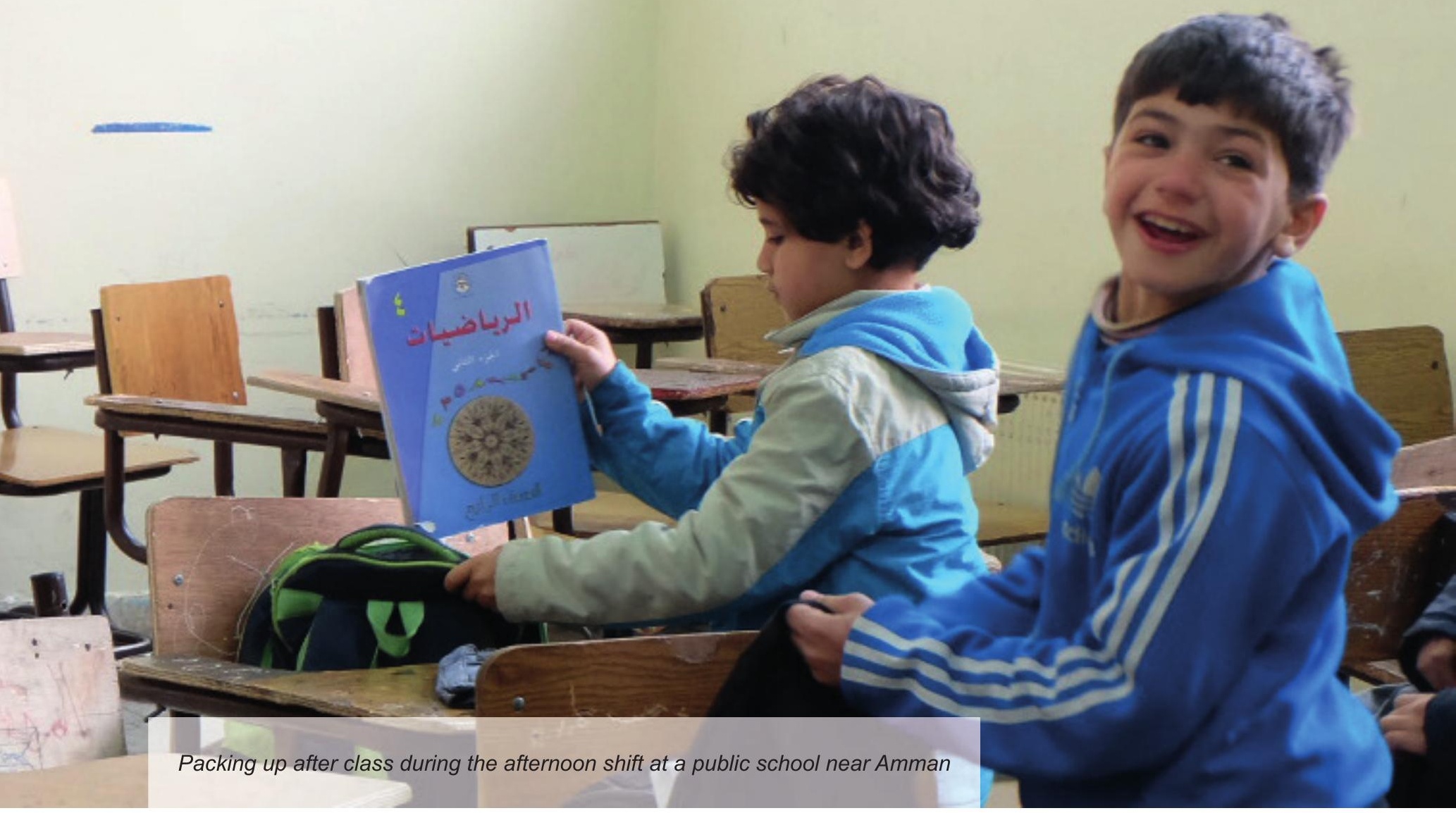

\subsection{Strengthen sectoral coordination and management}

As the EER continues to evolve and resilience and development become more important, the existing management capacities in the system are being challenged. However, there exists an effective and improving coordination system which should be strengthened. ESWG is underpowered for the task and there are weak mechanisms to maximise synergies, reduce duplication, secure economies of scale and share infrastructure; to share infrastructural, training and other HR costs; to coordinate procurement, to scale up effective practice, share data collection and pool capacities toward shared outcomes. Therefore this evaluation recommends the strengthening of the existing system of coordination rather than its radical restructuring. While one option would be to reduce the coordinating role of UNICEF and direct funding by donors to the MOE and NGOs, this would leave the education sector vulnerable to the diseconomies of fragmentation; UNICEF plays a key role in sustaining a platform of policies for sustainability. There are several specific recommended steps to strengthen coordination:

First, UNICEF should work with implementing partners and other stakeholders to ensure that the ESWG is adequately resourced and that staff are allocated sufficient time to participate fully in the working group and its related activities. Implementing partners also reported being too heavily committed to running programmes to have sufficient time to engage with the wider needs of the sector (in particular participating in the ESWG). In interviews, and from observations, staff throughout the sector appeared visibly tired, and staff turnover appeared to be further indication that the urgent might squeeze out the important. Opportunities for capacity building, training, information sharing and collaboration all struggled to compete with the urgency of delivering.

Second, UNICEF should work with implementing partners to ensure that standardised cost and performance data are available and used to make 
allocation decisions. Over time this should also promote greater competition between providers.

Third, as the role of the GOJ is crucial to successful coordination, the MOE should take a more active role in the ESWG and, along with UNICEF, should seek to ensure that projects build on demonstrated success. The MOE and MOPIC should help align education providers and should monitor the quality of education and use this to drive improvement. MOE and MOPIC are under considerable pressure to deliver existing programmes while being ready to respond to a rapidly changing environment. This requires a more clearly articulated medium-term vision.

Fourth, at the sectoral level, UNICEF should enhance its capacities (for example in relation to monitoring and evaluation) in order to adapt to changing circumstances. This has had particular consequences for efficiency, and the evaluation identifies several areas to address:

- The use of data is insufficient to maximise allocative and productive efficiency

- Only limited progress towards developing an outcomes oriented approach

- No value for money strategy in place

- Insufficient learning and agility to respond.

Finally, in support of the recommended mediumterm strategy, the MOE, MOPIC and UNICEF should create clearly articulated implementation plans. Current strategy documents often lack associated implementation and resource plans. Plans without priorities and implementation plans may not make the implications for donors and implementing organisations clear.

\subsection{Ensure flexibility and adaptability as circumstances change}

In the complex context of the Syrian conflict and the refugee crisis in Jordan, vital unknowns that affect planning are numerous. The evaluation team's perception is of a sector that is driven by events and by the pressing need to deliver interventions. The need to constantly react limits flexibility in response to new events. Key unknowns include not only the actual numbers of refugees in Jordan but also the time they are likely to spend in the country. In addition, resource availability from donors and government policies is uncertain. Given such uncertainty, planning needs to explicitly recognise these key unknowns. Robust planning would be supported by two kinds of thinking:

- Scenario planning to create plans relevant in multiple future scenarios. Scenario planning seeks to identify specific and achievable actions in different hypothetical but plausible futures, and is especially useful when there are significant uncertainties, but also strongly held values and commitments.

- A maturity model to agree what excellence might look like and how to approach it. A maturity model addresses where programmes are now, where they could be, and the incremental steps that would move them towards excellence.

\subsection{Develop a phased implementation plan with 'quick wins"}

There are many recommendations here, with different balances of suitability, feasibility and acceptability. Some are costly, and others involve issues about which stakeholders lack consensus. Therefore, this evaluation recommends a phased implementation plan, with rapid use made of 'quick wins', considered to be steps that could have the greatest impact most quickly during the 2015-2016 school year. Suggested quick wins include:

- Create additional double shifts to expand school spaces rapidly, accompanied by adding school days to the school year for double-shifted schools and outreach (MOE and UNICEF).

- An MOE policy for bullying in schools 
(developed by UNICEF), including teachers acting as school monitors before and after school and between shifts.

- Implement a policy of offering the MOE formal curriculum to age-appropriate clusters of out-of-school children (e.g. the first grade curriculum to 8 year olds in separate double shifts) (MOE and UNICEF).

- Donor prioritisation of school infrastructure and teacher salaries for formal education expansion, with a medium-term commitment 


\section{References}

Abuqudairi, A. 2014. 'Childhood lost: Syria's youth toil in Jordan.' Al Jazeera, 28 June. As of 16 September 2015:

http://www.aljazeera.com/news/ middleeast/2014/06/childhood-lost-syria-youthtoil-jordan-201462413920688310.html

AFAD. 2013. Syrian Refugees in Turkey, 2013. Field Survey Results. Republic of Turkey Prime Ministry: Disaster and Emergency Management Presidency.

Al-Daameh, M. 2013. 'Jordan, World Bank agree loan to assist kingdom with refugee influx'.

Asharq Al-Aswat. As of August 2016:

http://www.aawsat.net/2013/07/article55311567/ jordan-world-bank-agree-loan-to-assist-kingdomwith-refugee-influx

Altimari, M.N. 2014. Scoping Mission Report Jordan. HAP International.

Ammon. 2014. 'Prince El-Hassan: Syrian refugees crisis requires national-level thinking,' 7 December. As of 16 September 2015:

http://en.ammonnews.net/article. aspx?articleno=27251\#.VJICqV4BA

Bester, A. 2012. Results-Based Management in the United Nations Development System: Progress and Challenges. A Report Prepared for the United Nations Department of Economic and Social Affairs, for the Quadrennial Comprehensive Policy Review. Final Report July 2012. New York: UN. As of 16 September 2015: http://www.un.org/esa/coordination/pdf/rbm report 10 july.pdf

Binnendijk, A. 2011. Results Based Management in the Development Co-operation Agencies:

A Review of Experience. Background Report. Paris: DAC Working Party on Aid Evaluation, OECD.
Boyden, J. 2000. 'Conducting Research with War-Affected and Displaced Children: Ethics and Methods.' Cultural Survival Quarterly, Summer: 71-73.

Bray, M. 2008. Double-shift Schooling:

Design and Operation for Cost-effectiveness, Commonwealth Secretariat, London.

Chapman, R. 2011. Jordan Fiscal Reform Project II: Education Public Expenditures Working Paper, August 2011. USAID/Jordan Economic Growth Office. As of 16 September 2015:

http://www.frp2.org/english/Portals/0/PDFs/ Education\%20PEP\%20Working\%20Paper.pdf

Chatty, D., H. Ahmadzadeh, M. Çorabatır, L. Hashem, J. Al Husseini \& S. Wahby. 2014. Ensuring Quality Education for Young Refugees from Syria (12-25 years): A Mapping Exercise. Refugee Studies Centre, University of Oxford. As of September 2015:

http://www.rsc.ox.ac.uk/research/ensuringquality-education-for-young-refugees-from-syria

Davies, R. 2013. Planning Evaluability Assessments. A Synthesis of the Literature with Recommendations. DFID Working Paper 40.

DFID. 2011. DFID's Approach to Value for Money (VfM). As of 16 September 2015:

https://www.gov.uk/government/uploads/system/ uploads/attachment data/file/67479/DFIDapproach-value-money.pdf

DFID. 2014. Operational Plan 2011-2016: Jordan. December 2014. As of 16 September 2015: https://www.gov.uk/government/uploads/system/ uploads/attachment_data/file/389275/Jordan.pdf 
Education Sector Working Group. 2013. Joint Education Needs Assessment. As of June 2015: https://data.unhcr.org/syrianrefugees/download. php?id=2347

Education Sector Working Group. 2015. Jordan Minimum Standards for Education in Emergencies. INEE ESWG, Jordan.

Farrell, J.P. \& E. Schiefelbein. 1974. 'Expanding the scope of educational planning: The experience of Chile'. Interchange (5): 18-30.

Fuller, B., L. Dellagnelo, A. Strath, E. Santana Barretto Bastos, M. Holanda Maia, K. Socorro Lopes de Matos, A. L. Portela, and S. Lerche Vieira. 1999. 'How to Raise Children's Early Literacy? The Influence of Family, Teacher, and Classroom in Northeast Brazil'. Comparative education review 43(1): 1-35.

Graham, A., M. Powell, N. Taylor, D. Anderson \& R. Fitzgerald. 2013. Ethical Research Involving Children. Florence: UNICEF Office of Research - Innocenti.

Hashemite Kingdom of Jordan. 2014. Jordan Response Plan, October 2014. As of June 2015: http://www.jo.undp.org/content/dam/jordan/docs/ Publications/JRP+Final+Draft+2014.12.17.pdf

Healy, S. \& S. Tiller. 2013. 'A review of the humanitarian response to the Syrian refugee crisis in Jordan, 2012-13. Analysis of the emergency response capacity of the humanitarian system - Case study 3'. Medicins sans Frontieres. As of August 2016: www.msf.org/sites/msf.org/files/jordan case study final external.pdf

Healy, S., \& S. Tiller. 2013. 'Out of the Spotlight and Hard to Reach: Syrian Refugees in Jordan's Cities.' Humanitarian Exchange 59. As of 16 September 2015:

http://www.odihpn.org/humanitarian-exchangemagazine/issue-59/out-of-the-spotlight-and-hardto-reach-syrian-refugees-in-jordans-cities
Hugo, G. 2005. Migrants in Society: Diversity and Cohesion. Report prepared for the Global Commission on International Migration. As of 16 September 2015:

http://iom.ch/jahia/webdav/site/myjahiasite/ shared/shared/mainsite/policy_and_research/ gcim/tp/TP6.pdf

IMC \& UNICEF. 2013. Mental health/ psychosocial and child protection assessment for Syrian adolescents refugees in Za'atari refugee camp, Jordan. July 2013. Available online at: http://reliefweb.int/sites/reliefweb.int/files/ resources/IMC $\% 20$ MHPSS $\% 20$ and $\% 20$ CP $\% 20$ Assessment $\% 20$ Zaatari\%20July $\% 202013 \% 20$ final $\% 20(1)$.pdf

IMC \& UNICEF. 2014. 'Mental Health Psychosocial and Child Protection for Syrian Adolescent Refugees in Jordan'. As of August 2016: https://data.unhcr.org/syrianrefugees/download. php?id=7729

INEE. 2016. 'INEE Minimum Standards'. As of August 2016:

http://www.ineesite.org/en/minimum-standards

Inter-Agency Education Sector. 2014. 'Turkey: RRP6 Monthly Update-October. Education'. As of June 2015: http://reliefweb.int/sites/reliefweb. int/files/ resources/October2014dashboardEducation.pdf

Inter-Agency Working Group on Children's Participation (IAWGCP). 2007. Minimum Standards for Consulting with Children. Bangkok: IAWGCP. As of July 2014:

http://images.savethechildren.it/f/download/ Policies/st/standard-partecipazione.pdf

Jordan Education Sector Working Group. 2016. 'Terms of Reference'. As of August 2016: https://data.unhcr.org/syrianrefugees/download. php?id $=5678$ 
Jordan Times. 2015. 'Jordan preparing threeyear plan for refugee crisis - Fakhoury'. 23 March 2015. As of August 2016:

http://www.jordantimes.com/news/local/jordanpreparing-three-year-plan-refugee-crisis\%E2\%80\%94-fakhoury

Linden, T.. 2001. Double-Shift Secondary Schools: Possibilities and Issues. Washington, DC: World Bank..

Ling, T. 2012. 'Evaluating complex and unfolding interventions in real time.' Evaluation 18(1), 79-91.

MercyCorps. 2015. 'Against all odds: An equal opportunity to learn for refugee children with disabilities'. May 27 2015. As of August 2016: http://www.mercycorps.org/articles/jordan-syria/ against-all-odds-equal-opportunity-learn-refugeechildren-disabilities

Middle East and North Africa Out-Of-School Children Initiative \& UNICEF. 2014. Jordan Country Report On Out-Of-School Children. UNICEF MENA Regional Office.

Ministry of Education, Hashemite Kingdom of Jordan. 2015. 'Tawjihi results show slight rise in pass rate from last year.' As of 16 September 2015:

http://MOE.gov.jo/en/NewsDetails. aspx?NewsID=46

Moore, G., et al. 2014. Process Evaluation of Complex Interventions: Medical Research Council Guidance. London: MRC.

MOPIC \& UN in Jordan. 2004. The Millennium Development Goals Report (MDGR) for Jordan. As of 16 September 2015: http://www.jo.undp.org/content/jordan/en/home/ library/mdg/publication 2.html

MOPIC. 2013. Needs Assessment Review of the Impact of the Syrian Crisis on Jordan, 2013. MOPIC, Jordan.

MOPIC. 2014a. National Resilience Plan 20142016. MOPIC, Jordan.
MOPIC. 2014b. Jordan Response Plan 2015 for the Syrian Crisis: Final Draft. As of 16 September 2015:

http://www.jo.undp.org/content/dam/jordan/docs/ Publications/JRP+Final+Draft+2014.12.17.pdf

Morand, M., K. Mahoney, S. Bellour \& J. Rabkin. 2013. 'The implementation of UNHCR's policy on refugee protection and solutions in urban areas, Global survey 2012'. UN Refugee Agency. As of 16 September 2015:

http://www.unhcr.org/516d658c9.html

National Audit Office, UK. 2015. 'Principles of Good Commissioning.' As of 16 September 2015: http://www.nao.org.uk/successful-commissioning/ general-principles/principles-of-goodcommissioning/

National Center for Education Statistics. 2012. Trends in International Mathematics and Science Study. As of 16 September 2015:

https://nces.ed.gov/timss

No Lost Generation. 2014. No Lost Generation: One Year Report. September 2014. As of 16 September 2015:

http://childrenofsyria.info/download/5750/

Nusche, D. 2009. What Works in Migrant Education? A Review of Evidence and Policy Options. Paris: OECD. As of June 2015: http://www.oecd-ilibrary.org/content/ workingpaper/227131784531

OECD. 1986. 'Glossary of Terms Used in Evaluation', in Methods and Procedures in Aid Evaluation: A Compendium of Donor Practice and Experience. OECD: Paris.

OECD. 1991. 'The DAC Principles for the Evaluation of Development Assistance.' As of June 2015: http://www.oecd.org/dac/ evaluation/2755284.pdf

OECD. 2000. 'Glossary of Evaluation and Results Based Management (RBM) Terms.' As of June 2015: http://www.oecd.org/dac/2754804.pdf 
OECD. 2009. Education at a Glance 2009:

OECD Indicators. Paris: OECD. As of June 2015: http://www.oecd.org/edu/skills-beyond-school/ educationataglance2009oecdindicators.htm

OECD. 2015. 'DAC Criteria for Evaluating Development Assistance.' As of 16 September 2015:

http://www.oecd.org/dac/evaluation/ daccriteriaforevaluatingdevelopmentassistance. $\underline{\mathrm{htm}}$

OECD. n.d. The Paris Declaration on Aid Effectiveness and the Accra Agenda for Action (2005/2008). As of 16 September 2015: http://www.oecd.org/dac/effectiveness/34428351. pdf

Office of the High Commissioner for Human Rights. 1989. Convention on the Rights of the Child. As of 16 September 2015:

http://www.ohchr.org/en/professionalinterest/ pages/crc.aspx

Programme d'Analyse des Systems Educatifs des Etats et Gouvernements Members de la CONFENEM (PASEC). 2003. Le Programme de Formation Initiale des Maitres et de la Double Vacation an Guinee. Dakar: Conference des Ministres de l'Education (CONFENEM) des Pays Ayant le francais en Partage.

PhotoVoice. 2015. 'About Us'. As of 16 September 2015:

http://www.photovoice.org/about

Regional Refugee \& Resilience Plan (3RP). 2015. Regional Refugee \& Resilience Plan in Response to the Syrian Crisis. As of 16 September 2015: http://www.3rpsyriacrisis.org/

Save the Children Jordan. 2014. Back to School Campaign Report. June-October 2014. Save the Children Jordan.
Save the Children.2015. 'Resource Centre'. As of August 2015:

http://resourcecentre.savethechildren.se/library/ inter-agency-emergency-standard-operatingprocedures-prevention-and-response-gender-based

Stoianova, V. 2014. ICVA's Review of NGO's Experience with the Syria-Related Pooled Funds. International Council of Voluntary Agencies.

UN. 2013. 'Syria Regional Response Plan 6, Jordan'. As of 16 September 2015:

http://www.unhcr.org/syriarrp6/docs/syria-rrp6jordan-response-plan.pdf

UN. 2014. '2014 Syria Regional Response Plan, Mid-Year Update'. As of 16 September 2015: http://www.unhcr.org/syriarrp6/midyear/

UN. 2015. 'UN Education for All (EFA)'. As of 16 September 2015:

http://www.un.org/en/globalissues/briefingpapers/ efal

UNEG. 2005. UNEG Standards for Evaluation in the UN System. New York: UNEG. As of 16 September 2014:

http://www.unevaluation.org/papersandpubs/ documentdetail.jsp?doc id $=22$

UNEG. 2008a. UNEG Ethical Guidelines for Evaluation. March. New York: UNEG.

UNEG. 2008b. UNEG Code of Conduct for Evaluation in the UN System. New York: UNEG. As of July 2014:

http://www.unevaluation.org/document/detail/100

UNEG. 2011. Integrating Human Rights and Gender Equality in Evaluation - Towards UNEG Guidance. As of August 2016:

http://www.uneval.org/document/detail/980

UNESCO. 2011. Education Counts: Towards the Millennium Development Goals. As of 16 September 2015:

http://unesdoc.unesco.org/ images/0019/001902/190214e.pdf 
UNESCO. 2013. Syrian Refugees Education Country Profile Draft 2, 3/12/13, Jordan. UNESCO Jordan.

UNESCO. 2015. Education for Sustainable Development. As of 16 September 2015: http://www.unesco.org/new/en/education/themes/ leading-the-international-agenda/education-forsustainable-development/

UNESCO-IBE. 2011. UNESCO-IBE and UNESCO Institute for Statistics (UIS): Global Survey on Instructional Time - 2012. As of August 2016: http://www.ibe.unesco.org/fileadmin/user upload/ KPM/timedata2012/jordan infoavailable 2000.pdf

UNESCO Institute for Statistics. 2005. Children Out of School. Measuring Exclusion from Primary Education. As of 16 September 2015: www.uis.unesco.org/Library/Documents/oosc05en.pdf

UNESCO Office in Amman. 2016.. 'Education Management Information System'. As of August 2016:

http://www.unesco.org/new/en/amman/ education/ict-in-education/educationmanagement-information-system/

UNHCR. 2004. Executive Committee of the High Commissioner's Programme, Protracted Refugee Situations, UN High Commissioner for Refugees (UNHCR), EC/54/SC/CRP.14, 2004. As of 16 September 2015: http://www.unhcr.org/40ed5b384.html

UNHCR. 2009. UNHCR policy on refugee protection and solutions in urban areas. September 2009. As of 16 September 2015: http://www.unhcr.org/4ab356ab6.html

UNHCR. 2014a. 'Child Protection Sub Working Group - Jordan'. As of 16 September 2015: http://data.unhcr.org/ syrianrefugees/working group. php?Page $=$ Country\&Locationld $=107 \& \mid d=27$
UNHCR. 2014b. Child Protection Sub Working Group. Jordan: RRP6 Monthly Update - April. Protection. As of 16 September 2015: http://data.unhcr.org/syrianrefugees/download. php?id $=5873$

UNHCR. 2014c. Lebanon Education Update. December 2014. As of 16 September 2015: http://data.unhcr.org/syrianrefugees/download. php?id=8147

UNHCR. 2014d. Living in the Shadows. As of 16 September 2015:

http://unhcr.org/jordan2014urbanreport/homevisit-report.pdf

UNHCR. 2015a. 'Syria Refugee Response: InterAgency Information Sharing Portal.' As of 16 September 2015:

http://data.unhcr.org/syrianrefugees/country. php?id=107\#

UNHCR. 2015b. South Sudan Situation Emergency Response: Supplementary Appeal 2015. As of 16 September 2015: http://www.unhcr.org/54e747999.pdf

UNHCR. 2015c. 'Syria Regional Refugee Response.' As of June 2015:

https://data.unhcr.org/syrianrefugees/regional.php

UNHCR. 2015d. 'Syria Regional Refugee

Response. Jordan: Gender Equality.' As of 16

September 2015:

http://data.unhcr.org/

syrianrefugees/working group. php?Page $=$ Country\&Locationld $=107 \& \mid d=70$

UNHCR TRACKS. 2015. 'Picture this.' As of 16 September 2015:

http://tracks.unhcr.org/2014/10/picture-this/

UNICEF. 2010a. Core Commitments for Children in Humanitarian Action. New York: UNICEF. As of 16 September 2015:

http://www.unicef.org/cholera/Chapter 1 intro/05 UNICEF Core\%20Commitments for Children in Humanitarian Action.pdf 
UNICEF. 2010b. Working For an Equal Future. UNICEF Policy on Gender Equality and the Empowerment of Girls and Women. As of 16 September 2015:

http://www.unicef.org/gender/files/UNICEF Gender_Policy_2010.pdf

UNICEF. 2012. 'Support to the emergency education response for displaced Syrian children and host communities in Jordan.' ENPI 2012/297-963 \& ENPI 2013/321-638 (UNICEF REF. SC/2012/0378), second intermediate report prepared for The European Union.

UNICEF. 2013a. Access to Education for Syrian Refugee Children and Youth in Jordan Host Communities. Joint Education Needs Assessment Report.

UNICEF. 2013b. 'Protecting children from violence, exploitation and abuse.' As of 16 September 2015:

http://www.unicef.org/protection/57929 57972. $\underline{\mathrm{html}}$

UNICEF. 2013c. Shattered Lives: Challenges and Priorities for Syrian Children and Women in Jordan. Amman: UNICEF. As of 16 September 2015:

http://www.unicef.org/infobycountry/files/

Shattered Lives June10.pdf

UNICEF. 2013d. Syrian Refugee Children in Jordan: Assessments Desk Review on the Situation of Syrian Refugee Children Living in Jordan. UNICEF Jordan.

UNICEF. 2014a. Syria Crisis Monthly Humanitarian Situation Report, 14 July - 15 August 2014. New York: UNICEF.

UNICEF. 2014b. A Study on Early Marriage in Jordan. As of 16 September 2015: www.unicef.org/mena/UNICEFJordan EarlyMarriageStudy2014.pdf
UNICEF. 2014c. No Lost Generation - Protecting the Futures of Children Affected by the Crisis in Syria (Strategic Overview), ReliefWeb. As of 16 September 2014:

http://www.unicef.org/appeals/files/No Lost_Generation_Strategic_Overview January 2014.pdf

UNICEF. 2014d. UNICEF Annual Report $2013-$ Jordan. Amman: UNICEF. As of 16 September 2015:

http://www.unicef.org/about/annualreport/files/ Jordan COAR 2013.pdf

UNICEF. n.d. Makani: All Children in Jordan Accessing Learning (internal document).

UNICEF Evaluation Office. 2002. 'Children Participating in Research, Monitoring And Evaluation (M\&E) - Ethics and Your Responsibilities as a Manager.' Evaluation Technical Notes (1). April. As of 16 September 2014:

http://www.unicef.org/evaluation/files/TechNote1 Ethics.pdf

UNICEF Jordan. 2015. '”Makani' (My Space) initiative to expand learning opportunities for vulnerable children in Jordan'. 12 March 2015. As of August 2016:

http://www.unicef.org/jordan/media 10093.html

UNICEF Jordan Country Office. 2015. Guidance Note on 'Makani' - 'My Space' Approach (internal document).

UNICEF/REACH. 2014. Access to Education for Syrian Refugee Children in Zaatari Camp, Jordan. Joint Education Needs Assessment Report. As of September 2014: https://data.unhcr.org/syrianrefugees/download. php?id=7394

USAID. 2015. 'Syria - Complex Emergency. Fact Sheet \#4, Fiscal Year (FY) 2015'. As of August 2016:

https://www.usaid.gov/sites/default/files/ documents/1866/syria ce fs04 01-22-2015.pdf 
Vähämäki, J., M. Schmidt \& J. Molander. 2011. Review: Results Based Management in Development Cooperation. Riksbankens Jubileumsfond, Stockholm

Wash Emergency Assistance In Jordan Schools. 2014. Report On Activities From Phase I: Assessment Of WASH Facilities In Schools. UNICEF \& JEN.

WASH Working Group. 2014. Jordan: RRP6 WASH Sector Achievements January to September 2014. As of 16 September 2015: http://data.unhcr.org/syrianrefugees/download. php?id=7141

Watkins, K., \& S.A. Zyck. 2014. Living in Hope, Hoping for Education. London: Overseas Development Institute.
World Bank. 2010. Education Reform for Knowledge Economy I Program: Overview. As of 16 September 2015:

http://www.worldbank.org/projects/P075829/ education-reform-knowledge-economyprogram?lang=en\&tab=overview

World Bank. 2014. Learning in the Face of Adversity, The UNRWA Education Program for Palestine Refugees, Conference Edition: October 2014. As of June 2015:

http://unispal.un.org/

UNISPALNSF/0/48E2EA199AE 1317E85257D8F005759A6

World Commission on Environment and Development, 1987. Our Common Future. Oxford: Oxford University Press. As of 16 September 2015:

http://www.un-documents.net/wced-ocf.htm 



\section{Annex 1: Evaluation questions and summary findings}

\section{Relevance}

Evaluation question

Overview of findings

How strategies target needs

Relevance and appropriateness in responding to needs of displaced Syrians and vulnerable Jordanians

Responses to unforeseen contingencies, and timeliness when coordinating with other agencies

Strategy contribution to formal education and supporting services

- Contribution to policy dialogue

- How strategies target needs of service providers

- Alignment of Jordanian, UN and wider regional strategies

- $\quad$ Alignment of donors and partners

Evaluation

Achievement of goals in RRP6, 3RP and relevant government plans

Equitable access to appropriate education
Activities in 2012-2013 were highly relevant, creating a rapid response to educational needs enabling many displaced Syrian children to enrol in school with supporting services. The scope and protracted nature of the crisis were not foreseen. As time passed, strategic development did not keep pace with events.

While there are high-level documents (RRP6 and 3RP) that describe objectives, needs and activities, there is no single document that explicitly lays out a strategy or Theory of Change for the education response, formal education and supporting services.

The GOJ, donors, UNICEF and service providers developed structures to discuss policies, as well as the overarching strategy documents RRP6 and 3RP. Strategy development, contribution to policy dialogue, alignment with wider regional and national strategies, and alignment of donors and partners (GOJ, UN agencies, and services providers) were influenced and constrained by: complex, interacting components; rapidly changing circumstances; lack of consensus on time frame and approach to what is relevant in the longer term; being relevant to a largely urban crisis in a middle-income country; and tensions between a nationalitybased approach and a vulnerability or rights-based approach. These factors posed challenges to adapting the approach and activities in the face of changing circumstances and needs.

\section{Effectiveness}

\section{Objectives stated in RRP6 and 3RP included access to education, quality and protection of education and meeting the needs of vulnerable Jordanians. Findings for each are summarised in the lines below.}

Providing access to formal education for 130,000 Syrian children is a significant accomplishment of the education response. However, having at least 60,000-97,000 Syrian children out of school requires urgent action. Improving access depends on understanding determinants of access that include location, characteristics of education provision, and characteristics of the family and child. 
Learning environments promoting quality

Meeting the needs of vulnerable Jordanians and

Syrians

Strategic use of alternative education

Quality has been a secondary consideration to access. While Jordan has a robust school system, quality of education provided to the Syrian refugees has been below desired levels. Many of the quality challenges have affected Jordanians as well as refugees. While there are few ways of measuring quality, it is known that: test scores are low; classrooms are crowded; teachers struggle in difficult environments; facilities are in need of maintenance; Syrian students struggle in making the transition from Syrian to Jordanian curricula; there are inconsistent policies about grade placement; and unclear pathways to adulthood demotivate students. On the one hand, double-shifting has provided opportunities to meet the quality needs of the Syrians and Jordanians, but on the other double shifts in Jordan work worse than elsewhere.

Additional influxes of students into Jordan's formal system have crowded out resources for quality improvements for Jordanians, and classroom conditions have become more crowded. Vulnerable Jordanians feel that they receive less attention than vulnerable Syrians.

Alternative education has been provided for 35,000 Syrian refugee children, and providing such access has been more effective in camps than in host communities. Alternative provision is perceived to provide high-quality, relevant, child-centred and flexible education to Syrians. However, there is a lack of a clear framework for what alternative education is intended to achieve over the longer term or consistent monitoring of quality, meaning that standards and achievements are hard to measure.

\section{Efficiency}

Evaluation question

Identifying needs and developing services to address them, including how efforts were designed to meet learning needs assessments

Justifying budgets and securing funding

Coordinating and aligning activities

\section{Overview of findings}

Efficient allocation of resources within the Programme is linked to the identification of needs. Data were often relevant, sufficient and credible. However, further improvements are required to ensure that allocation of resources are prioritised to reflect the distribution of needs. Additionally, there appears to be no systematic options appraisal to ensure that the activities are designed to meet needs as efficiently as possible. There were no learning needs assessments available for the Syrians in Jordan.

Planning tools, as well as fund-raising tools, such as the RRP6 and 3RP, NRP 2014-2016 and JRP 2015 are limited by several factors including: cynicism regarding the amounts of funding requested, the view that it was not prioritised, and perceived lack of transparency for donors. Planning documents could be strengthened to mobilise funding and create a transparent account to donors. Donors, in response, should consider stable long-term funding to support longer-term investment in efficiencies.

UNICEF has played a positive role in supporting the ESWG at the sectoral level, and the GOJ at the national level, in achieving alignment of specific activities with overall strategic goals. The ESWG requires further support and re-energising while tensions remain between IPs and the MOE. Given the rapidly evolving situation this is understandable, but focusing coordination on efficiency improvements should be a high priority especially as the EER seeks greater focus on resilience and quality of provision. 
Procurement policies, controlling costs and delivering value for money

Delivering outcomes-focused management and a results-based approach

Using monitoring and evaluation to drive improvement and build capacity

Based on available data it is not possible to arrive at cost effectiveness analysis; producing such data should be the focus of future efforts. Many components required to deliver value for money are either in place or being developed, and these should be brought together and communicated across the EER within an overall strategy. UNICEF should embrace a more analytical approach to identifying and communicating steps required to embed VfM within existing activities. UNICEF lacks the data needed to demonstrate that the EER delivers value for money.

Overall, the relationship between UNICEF and implementing partners has been focused on transactions rather than outcomes. The short-term aim should be to improve the understanding of activity costs, but over time efforts should focus more on outcomes.

Data has been 'pulled through' into decisionmaking. There has been improving data on implementing partners' activities, and examples of learning and adaptation. However, a more effective system for measuring outcomes and quality is needed for the programme to move forward. In general, feedback and learning mechanisms were weak or underresourced, and outcome data is used only to a limited extent.

\section{Cross-cutting}

\section{Evaluation question}

Governance and coordination address cross-cutting issues

Meeting the needs of both genders

Promoting a protective learning environment and integrating a strategic approach to child protection

Coordination with health/nutrition and WASH

In line with Core Commitments for Children and human rights-based approach in programming

\section{Overview of findings}

Cross-sectoral collaboration has strengthened significantly over time thanks to investment in coordination structures and key initiatives. However, increased investment is needed to address gaps in operational synergies among EER partners and to expand activities addressing needs related to the full range of cross-cutting issues and sectors.

Efforts around gender mainstreaming and capacity building as well as enhanced coordination and disaggregated reporting show that gender is a Programme priority. However, crucial gaps remain and programmatic focus is still overwhelmingly on outreach and support for girls, undervaluing the specific and severe challenges boys are facing.

A lack of adequate psychosocial support in schools and unsafe environments mean that some children face severe risks. Promising initiatives such as Makani centres show a coherence of purpose between sectors and the capacity for coordinated action and innovation in this area; however strategic action is needed to address gaps in MOE schools in particular.

Effective practice efforts to build government capacity around WASH in schools and develop gender-appropriate facilities and infrastructure have had impressive reach and show coherence of approach and priorities between sectors. However, infrastructural problems persist in some schools, and in others, WASH facilities fall short on cleanliness. Health interventions and assessments in schools have reached many children. However, enhanced coordination is needed as access to healthcare remains a challenge and some children go to school hungry.

While Programme efforts have been impressive, with so many out of school and with compromised learning environments, it is ultimately falling short of fulfilling the Core Commitments for Children. In addition, a number of rights challenges remain related to gender and children with disabilities, as well as health and nutrition, child protection and WASH. 


\section{Sustainability}

Evaluation question

Factors that influence sustainability of programme outcomes

Contribution to national institutional capacity building

UNICEF's efforts to ensure that UNICEF staff and implementing partners had necessary capacity and expertise

How community voices have been fed into programming and the extent to which the EER contributes to empowerment of the Syrian community

\section{Overview of findings}

Factors that are important for ensuring continuity of education into the future include: making trade-offs; advocacy, policy dialogue and coordination; capacity building; funding; and community engagement. To date trade-offs in the face of time and resource constraints have not been explicitly acknowledged and should be a key part of planning. There has been significant policy dialogue and coordination; donors and UN agencies could make better use of their influence and pool advocacy resources. Funding plans to date have been short term, and require creative longer-term thinking.

Capacity building has started, but there are further needs to build MOE management capability, sustainably pay teachers, build infrastructure, and expand MOE quality assurance programmes for schools.

UNICEF responded to the refugee influx with expansion of human resources and staff skills. UNICEF have also invested in implementing partners, improving their systems and providing trainings, reflecting key priorities such as M\&E. Staff turnover and the preservation of institutional knowledge at UNICEF has been a challenge for UNICEF staff, highlighting the need for UNICEF management systems to be adaptable and agile enough to respond to the developing context in Jordan.

While the inclusion of Syrian and Jordanian community voices was not prioritised from the outset, interviews reflect stakeholders' acknowledgement of its importance going forward, for both community cohesion and appropriateness and sustainability of interventions. Existing initiatives such as the use of Syrian assistant teachers and inclusive approaches to alternative learning offer promising examples to build upon in this regard. 


\section{Annex 2: Further detail on methodology}

While methodology for this evaluation was summarised in the Introduction, the sections below provide further detail on particular aspects of the methodologies used.

\section{Sampling and geographical coverage}

The table below details sampling and geographical coverage by each method used in this evaluation:

Table A2.1: Methods, sampling and geographical coverage of the evaluation

\begin{tabular}{|c|c|c|}
\hline Method & Sample and further details & Geographical coverage \\
\hline Document review & $\begin{array}{l}\text { Academic literature, historical practice review, UNICEF } \\
\text { internal documents, and documents from partners }\end{array}$ & $\mathrm{n} / \mathrm{a}$ \\
\hline $\begin{array}{l}\text { Education stakeholder } \\
\text { interviews }\end{array}$ & $\begin{array}{l}39 \text { interviewees }{ }^{131} \text { with GOJ (MOE and MOPIC), UN } \\
\text { officials (UNHCR, UNICEF, UNESCO), donors (EU, } \\
\text { Canadian Development Aid and US Department of } \\
\text { State's Bureau of Population, Refugees, and Migration), } \\
\text { local and international NGOs, community and religious } \\
\text { groups operating in camp and urban settings, municipal } \\
\text { education authorities, education managers in the } \\
\text { Zaatari camp and Azraq camp, and four principals of } \\
\text { schools with a significant number of Syrian refugees as } \\
\text { students. }\end{array}$ & Amman, Zarqa, Azraq, Zaatari \\
\hline $\begin{array}{l}\text { Focus groups with } \\
\text { children, parents and } \\
\text { teachers }\end{array}$ & $\begin{array}{l}62 \text { focus groups. } \\
\text { The sample was evenly distributed in gender and } \\
\text { age groups. Purposive sampling of different groups } \\
\text { was used, with participants selected for their ability to } \\
\text { provide information about different contexts. This large } \\
\text { sample size and diverse sampling technique enabled } \\
\text { capturing a wide range of perspectives relating to } \\
\text { educating Syrian refugee children common themes, and } \\
\text { variations. }\end{array}$ & $\begin{array}{l}\text { In Zaatari and Azraq camp } \\
\text { focus groups included } 119 \\
\text { children, } 23 \text { parents and } 18 \\
\text { teachers/providers. } \\
\text { In host communities (in Irbid, } \\
\text { Amman, Zarqa, Karak and } \\
\text { Mafraq) focus groups included } \\
149 \text { children, } 28 \text { parents and } 28 \\
\text { teachers/service providers. }\end{array}$ \\
\hline $\begin{array}{l}\text { Participatory } \\
\text { photography }\end{array}$ & $\begin{array}{l}\text { Children aged } 12-17 \text { ( } 11 \text { children in Zaatari camp and } \\
36 \text { children in host communities). }\end{array}$ & $\begin{array}{l}\text { Children involved in } \\
\text { participatory photography were } \\
\text { from Zaatari camp and host } \\
\text { communities (Amman, Mafraq, } \\
\text { Zaraq, Karak, and Irbid). }\end{array}$ \\
\hline
\end{tabular}

131 Government of Jordan (MOE and MOPIC), UN officials (UNHCR, UNICEF, UNESCO), donors (EU, Canadian Development Aid and US Department of State's Bureau of Population, Refugees, and Migration), local and international NGOs, community and religious groups operating in camp and urban settings, municipal education authorities, education camp managers, and four principals of schools with a significant number of Syrian refugees as students. 


\begin{tabular}{|c|c|c|}
\hline Method & Sample and further details & Geographical coverage \\
\hline $\begin{array}{l}\text { Secondary analysis } \\
\text { of Zaatari and host } \\
\text { community Joint } \\
\text { Education Needs } \\
\text { Assessment (JENA) } \\
\text { survey data. }\end{array}$ & $\begin{array}{l}\text { The Zaatari Camp data included a relatively small } \\
\text { sample size, with a consequently smaller sample size } \\
\text { for particular groups. However, the sample size was } \\
\text { sufficiently large to allow multivariable analysis as a } \\
\text { predictor of attendance in formal education. Zaatari } \\
\text { Camp Needs Assessment used random sampling of } \\
\text { households with children aged } 3-18 \text { based on GPS } \\
\text { coordinates (sample size: } 1,125 \text { children). } \\
\text { In the host community data, data collectors took a } \\
\text { non-probability approach to the sampling of Syrian } \\
\text { households, based on a sampling frame from the } \\
\text { World Food Programme, a key-informant network, and } \\
\text { snowball sampling of households within communities } \\
\text { (sample size: } 9,085 \text { children from } 3,327 \text { households). }\end{array}$ & $\begin{array}{l}\text { Zaatari Camp } \\
\text { Host communities (Ajloun, } \\
\text { Amman, Aqabah, Balqa, Irbid, } \\
\text { Jarash, Karak, Maan, Madaba, } \\
\text { Mafraq } \\
\text { Tafilah and Zarqa) }\end{array}$ \\
\hline
\end{tabular}

\section{Preparatory work for participatory photography}

In order that participatory photography could be incorporated into some of the focus groups, the evaluation team delivered cameras to the children and conducted training on use and the themes for the pictures. The evaluation team developed the pictures prior to group discussions where the facilitator asked children to select the pictures most relevant to them. The pictures then framed the discussion and the questions. The evaluation team selected pertinent photographs from the children's collection and have interspersed these throughout the report.

\section{Limitations to the focus group and survey data analysis methodologies}

To conduct the qualitative interviews and collect the focus group data, a purposive sample was used to enable the evaluation team to understand experiences and develop a theory. However, there are several limitations to this purposive sampling method:
- Partner organisations recruited participants, possibly leading to sample bias. However, the evaluation sample size and diversity enabled review of a wide variation in perceptions.

- Focus group interviews were conducted on partner organisation premises, but interviewers established rapport and used various inquiry methods to minimise bias.

Limitations to the JENA data collections available to the evaluation for both Zaatari Camp and for host communities. ${ }^{132}$

- The Zaatari Camp data included a relatively small sample size, with a consequently smaller sample size for particular groups. However, the sample size was sufficiently large to allow multivariable analysis as a predictor of attendance in formal education.

- In the host community data, data collectors took a non-probability approach to the sampling of Syrian households. This is not an appropriate approach for formal statistical inference; however it was a pragmatic and practical approach to identifying the relevant population given the context. 
- There are limitations associated with the questions about non-formal and informal education, as lack of clarity in data collection rendered it is impossible to distinguish between among responses.

- Definitions of key terms were not used correctly in questions; in particular 'enrolment' and 'attendance' were used in improper contexts.

- Concerns were also raised about the reliability of the work of enumerators in the field.

\section{Ethical issues}

RAND's research for this evaluation was designed, reviewed and conducted in a way that ensures adherence to a set of Research Ethics Principles, established in order to protect the interests of research participants and researchers and to maintain the integrity and objectivity that are central to the corporate mission and values. RAND's ethical guidelines were complemented by guidelines set out by the United Nations Evaluation Group (UNEG) ${ }^{133,134,135}$ and reinforced by the principles laid out in guidelines for conducting research involving children. ${ }^{136}$, 137, 138, 139, 140 The evaluation team took concrete measures to ensure that the focus groups with parents, providers, boys and girls are carried out in a way that upholds best ethical practice to protects the interests of participants. The HSPC is charged with ensuring the ethical treatment of individuals who are participants in all of the corporation's projects, and serves as the Institutional Review Board to review research involving human subjects.

The evaluation team worked closely with UNICEF throughout the process to maintain an understanding of stakeholders' perspectives, which were collected primarily through interviews and documentary reviews.

\section{Human rights and gender equality}

The evaluation adheres to the UN principles for integrating human rights and gender equality in evaluation. ${ }^{141}$ Accordingly children, parents and teachers were included in the data collection methods described above and PhotoVoice methods were selected specifically because they are especially well-suited to understanding children's perspectives. Focus groups were also designed to engage boys and girls differently. The process was therefore participatory and inclusive.

The evaluation collected data to understand both men and women, and boys and girls. Field visits included both girls schools and boys schools and both parents and teachers were interviewed or participated in focus groups. The use of mixed methods as described above also coheres with the UN guidance on good practice.

The EER Programme is focused on realising the rights of children to an education and therefore assessing the success of the Programme is directly relevant to the UN's role in protecting human rights and gender equality
UNEG (2008a)

UNEG (2005).

UNEG (2008b)

UNICEF (2010a).

Graham et al. (2013)

IAWGCP (2007).

UNICEF Evaluation Office (2002).

Boyden (2000).

UNEG (2011). 



\section{Annex 3: Summaries of challenges, effective practices and remaining needs in key areas of the evaluation}

Table A3.1: Summary table - relevance

\section{Relevance challenges} components

- Rapidly changing circumstances

- Lack of consensus on time frame and approach to what is relevant in the longer term

- Being relevant to a largely urban crisis in a middleincome country

\section{Effective practice addressing \\ Remaining needed steps} needs

- Key documents that include the RRP6, 3RP, NRP and JRP

- Targeting urgent needs early in the crisis

- A shift in the field to beginning to address longer-term concerns through programmes stakeholders
- Develop an explicit Theory of Change and medium-term strategy, with buy-in from

- Ensure strategic adaptation over time as circumstances change

- $\quad$ Prioritise planning and roadmap to align high-level goals with programmes

- $\quad$ Shift from specific programmes to a system-wide approach

- $\quad$ Shift to more appropriate programming in urban areas in addition to camps

- Implement vulnerability-based approaches instead of nationalitybased approaches 
Table A3.2: Summary table - effective provision of access to formal education opportunities

\section{Effectiveness challenges affecting access}

- $\quad$ Remaining large numbers of Syrian and Jordanian out-ofschool children

- Enrolment policies inhibit out-ofschool children from registering for formal education

- Absence of a formal education programme for children aged 6-12 who have been out of school for several years

- No 'map' available of school spaces, need for school spaces, and transportation needs to inform planning, with tailored plans per governorate

- Insufficient part-time tailored programmes for child labourers and married children

- Patchy security at schools, between shifts, during transportation

- Limited suitable opportunities for formal programmes for children who are behind their age group, for example, the formal curriculum offered at older ages

- Overcrowded schools lack materials and spaces

- Lack of transportation inhibits attendance in camps and host communities

- Parents lack awareness and information about education

- Prohibitive costs of transport and materials needed to attend school

\section{Effective practice addressing} needs

- GOJ opening up Jordanian formal schools to Syrians

- Second shifts expanded school spaces

- Outreach enrolment and retention campaigns

- Timely opening of schools in Zaatari and Azraq

- Upgrading and building schools, investing in infrastructure

- Catch-up programmes and remedial programmes to get children in school who have missed school, as well as the supports that keep them there, such as home-schooling

- Exploration of mechanisms to enable families to survive without child labour (e.g. parental employment, conditional cash transfers)

- Provision of education kits addressed need for basic materials

\section{Remaining needed steps}

- $\quad$ Build more schools in camps and host communities to increase capacity

- Expand double shifts to increase classroom spaces

- Continue support for the EMIS school mapping in order to effectively target resources for expansion per governorate

- Focus resources on geographical areas with the lowest rates of attendance (in camps and host communities) and work to identify the areaspecific barriers to attendance

- Invest in transport provision for areas with limited education services, in camps and urban areas

- Ensure all transport options are safe for girls and boys and work with school staff to address security issues at school

- Continue advocacy and strategic efforts to provide full-time, certified education with pathways for younger children who cannot enter an age-appropriate formal classroom

- $\quad$ Provide more education kits to families to limit costs associated with going to school

- Continue and expand enrolment and information initiatives to ensure that parents and children are aware of their education options

- Expand initiatives which allow child labourers and young mothers to attend school 
Table A3.3: Summary table - effective approaches to improve the quality of formal education provision

\begin{tabular}{l|l}
$\begin{array}{l}\text { Effectiveness challenges } \\
\text { affecting quality }\end{array}$ & $\begin{array}{l}\text { Effective practice } \\
\text { addressing needs }\end{array}$
\end{tabular}

\section{Monitoring quality}

- JENA a limited resource in terms of data quality and scope

- Lack of consistent, systematised monitoring of quality in schools, especially in second shifts

- Insufficient monitoring of quality indicators that reflect attendance, teaching methods, learning outcomes, teacher training, development and performance

- Not all implementing partners monitor education activities to ensure alignment with MOE policies and guidelines; too few appropriately trained staff

- Workplans for measuring quality are sporadic due to emergency status

\section{Systemic and institutional issues}

- No clear, established minimum standards

- Short school days and reduced instructional time in double-shifted schools

- Class sizes at unmanageable levels in places and overwhelmed

- Poor learning environments in camps

- Inadequate resources for facilities, training for inexperienced teachers, especially in second shifts

- Unequal and inadequate employment terms for second-shift teachers

- Syrian assistant teachers under-used

- Remaining differences in curriculum and certification which make it difficult for students and teachers to adapt

- Under-developed, inconsistent policies about grade placement for children

- Unclear pathways into adulthood and self-development

- $\quad$ Limited programmes to address needs of children who were out of school several months or more

- Too few special programmes (e.g. remedial classes) to prepare students for Tawjihi and improve pass rates of the Tawjihi secondary exit exam for Syrians

\section{Monitoring quality}

- Introduced JENA to identify education needs

- Supported the implementation of EMIS

- Some monitoring from implementing partners

\section{Systemic and institutional} issues

- $\quad$ Agreed INEE/ESWG minimum standards for quality

- MOE providing national leadership with a strategic plan

- UNICEF and ESWG provided coordination and timely support to MOE

- Sustained engagement of international donor community

- Developed an agreed approach to bullying and violence

- MOE schools expanded infrastructure capacity rapidly

- Renovations of schools to improve facilities

Using double-shifting to reduce classroom crowding and tailor classes to the needs of a refugee population

- Use of Syrian assistant teachers to help in the classroom, provide homework support and reduce bullying

- $\quad$ Teacher training delivered for classroom management

\section{Remaining needed steps}

\section{Monitoring quality}

- Expand MOE capacity to monitor quality in formal education

- Invest in training of school staff MOE officials and NGO partners to monitor quality indicators

- Conduct and expand regular and comparable JENA surveys in camps and host communities

\section{Systemic and institutional issues}

- Invest in advocacy to encourage an emphasis on quality of education in the formal system, including in second shifts

- Ensure the development and dissemination of quality guidelines, based on INEE standards

- Invest in advocacy and training around the INEE standards

- Develop and disseminate guidance for teachers and principals which explicitly recognises refugee needs

- Ensure full-time education with instructional time in accordance with international averages in all schools

- $\quad$ Continue to invest in expanding spaces through building and double-shifting targeted at areas most in need

- Ensure fair and sustainable working terms and salaries for all teachers, regardless of shift

- Expand the use of Syrian assistant teachers in camps and host communities to improve learning environments and understanding

- $\quad$ Expand and improve teacher training programmes to manage diverse classes and differences in curricula

- Develop clear, consistent national policy on grade placement and progression

- Invest in and expand remedial and other supportive programmes for students who need special assistance and attention 
Table A3.4: Summary table - addressing the needs of children excluded from formal education opportunities accessing alternative learning activities in community and camp settings

\section{Effectiveness challenges -} alternative learning

- $\quad$ No clear basis for prioritising which programmes to invest in given the multiple needs

- Lack of coordinating pathways between formal and alternative education

- Lack of certification to provide routes from education to employment

- Lack of unified curriculum and quality standards

- $\quad$ Lack of adequate instructional time

- Restrictive enrolment policies

- Poor data to compare instructional time, learning outcomes and costs of alternative and formal programmes

- Absence of non-formal provision for those aged 6-11

- Lack of adequate referral pathways from other services in host communities inhibit the identification of out of school children

\section{Effective practice addressing} needs

- Makani centres: a promising approach to integrating psychosocial services alongside education services

- Emerging framework for alternatives to formal education (associated with the planning for Makani) $)^{143}$

- Developing programmes for multiple needs

- Developing inclusive programmes for Syrians and Jordanians

- Fast response in scaling up to the crisis

- $\quad$ Perceived quality of teaching and programming among parents and children

- Development of coordinated curricula for some programmes

- Use of Syrian staff
Remaining needed steps

- Invest in an evidence-based strategy to prioritise programmes to invest in

- Take the lead on advocacy with MOE and donors to establish coordinated pathways and progression between alternative education and formal education

- Introduce certification to provide routes to future learning and employment

- Design and implement a unified, validated curriculum for efficiency and quality standards

- Provide formal or non-formal programmes for younger children who have been out of school for 3 or more years

- Ensure adequate instructional time wherever possible

- Develop an age-appropriate non-formal system that caters to those under 12 years old

- Lead coordination of referral pathways from other sectors and services to ensure out of school children are identified, especially in host community settings

- Establish consistent quality standards, indicators and monitoring

- Improve data collection so that data on instructional time, learning outcomes, and costs of alternative programmes and formal programmes is available for comparison 
Table A3.5: Summary table - efficiency

\section{Efficiency challenges}

- Allocative efficiency (delivering goods and services that accurately reflect the balance of need)

- $\quad$ The MOPIC requirement that at least $30 \%$ of the targeted population are Jordanians is determined by the need to strengthen acceptability but it lacks any evidence as a basis for allocative efficiency

- $\quad$ Productive efficiency (producing goods and services to the required standard for the lowest cost)

- $\quad$ There are no systematic options appraisal processes in place to ensure that the activities are designed to meet needs as efficiently as possible

- In a rapidly evolving sector with multiple players the coordinating capacity of ESWG, for example, is under-powered to ensure that efficiency is achieved through reducing duplication, learning lessons from good practice, pooling training resources and so forth.

- There is a limited understanding of the meaning of Value for Money, the steps required to deliver this, and consequently no systematic value for money strategy.
Effective practice addressing needs

- $\quad$ Processes in place for ensuring a balanced portfolio of activities in the education sector based upon reliable evidence of needs and available means to meet these needs

- Good examples of needs analyses being used to shape services

- Data should be much improved by the implementation of the UNESCO OpenEMIS software in Jordan.

- Cost data are available and used to drive efficiency and outcome data are used to identify examples of efficient delivery which are then spread to others

\section{Remaining needed steps}

- Improve planning to ensure that allocation of resources are prioritized to reflect the distribution of needs.

- $\quad$ Further develop key planning documents to support needs analyses to limit cynicism about their accuracy and completeness

- Utilise EMIS software to support efforts to better understand outcomes and in particular to measure what value is delivered through educational services (including the quality of the services provided) and to target benefits equally based on these data.

- $\quad$ Strengthen advocacy efforts with MOPIC to to ensure that policy supports allocative efficiency

- Develop systematic options appraisal processes to ensure that activities are designed to meet needs as efficiently as possible

- $\quad$ Utilise comparative cost data to identify unjustified variations and thus the lowest possible costs. Standardised costs would be an effective way to help achieve this and this could be written into agreements between UNICEF and implementing partners.

- Ensure adequate coordination via the ESWG for efficiency through reducing duplication, learning lessons from good practice, pooling training resources and so forth.

- Build capacity and knowledge around Value for Money, the steps required to deliver this, in order to move towards a systematic value for money strategy.

- Focus in the short term on the efficient delivery of outputs (e.g. the cost per 'classroom hour'), but over time shift to an emphasis on outcomes (the quality of education outcomes). Transactions (e.g. to maintain trust, share understanding, appreciate challenges faced by other partners) are important but should not be pursued at the expense of an outcomes focused approach. 


\section{Summary tables - cross-cutting issues}

\section{Table A3.6: Summary table - gendered differences and approaches}

\section{Challenges specific to gender}

Girls

- Early marriage and pregnancy

- Confinement to the home

- Verbal mistreatment in schools

- SGBV

Boys

- Lower school attendance

- Greater rates of child labour

- Family responsibilities

- Violence and abuse in and around school

- $\quad$ Separation from parents following police crackdowns

- Demotivation and frustration in school

- Poorer behaviour and performance in school

- Recruitment to armed groups

- Preference for vocational training

\section{Effective practice addressing} needs

- Integrating gender equality into planning documents

- Cross-sectoral gender focal points

- Gender-disaggregated data

- Community engagement around child marriage

- Programmes for girls to continue education after marriage

- Gender-appropriate WASH facilities in camps and host communities

- Cash transfers for child labourers to attend school

\section{Remaining needed steps}

- $\quad$ Extend programmes for married girls and young mothers to return to education

- Continue work with communities and parents to change attitudes to girls' education

- $\quad$ Provide additional gendered programming targeted at boys

- Offer programmes to reduce child labour

- Deliver educational programmes for child labourers

- $\quad$ Provide teacher training and monitoring to address mistreatment in schools

- Improve security around schools and anti-bullying programmes

Table A3.7: Summary table - children with special needs and disabilities

Challenges specific to children with disabilities

- Social stigma

- Transport and distance to school

- Teacher capacity

- Lack of national policy to direct schools and integrate children with disabilities

- Lack of systematic referral systems

- $\quad$ Lack of reporting by partners on children with disabilities

- Short-term funding cycles and approval periods

\section{Effective practice addressing} needs

- Inclusive education training manual for teachers

- Decentralised training programme for teachers

- Shadow teachers

- Efforts to establish crossgovernment strategy and dialogue

\section{Remaining needed steps}

- $\quad$ Strengthen advocacy and capacity building activities for a national strategy on inclusive education for children with disabilities

- Take the lead on coordination for improved referral mechanisms between all relevant actors on the field

- $\quad$ Ensure all implementing partners disaggregate reporting on children with disabilities

- Focus on long-term capacity building and policy development to address needs of Jordanian and Syrian children with disabilities 
Table A3.8: Summary table - health and nutrition

\begin{tabular}{|c|c|c|}
\hline $\begin{array}{l}\text { Challenges specific to health and } \\
\text { nutrition }\end{array}$ & $\begin{array}{l}\text { Effective practice addressing } \\
\text { needs }\end{array}$ & Remaining needed steps \\
\hline 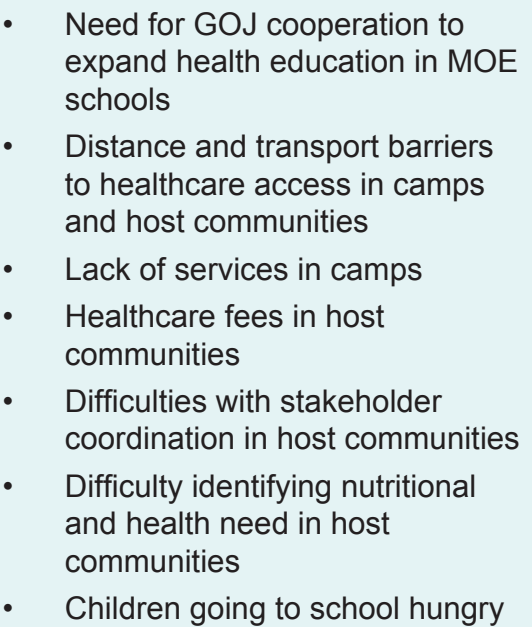 & $\begin{array}{l}\text { - Systematic school-level health } \\
\text { assessments and vaccinations in } \\
\text { schools and camps } \\
\text { - Inclusive healthcare } \\
\text { interventions in alternative } \\
\text { centres }\end{array}$ & $\begin{array}{l}\text { Improve coordination to fill } \\
\text { data and service gaps in host } \\
\text { communities } \\
\text { - Provide of transport to improve } \\
\text { access to health services in } \\
\text { camp and host communities } \\
\text { Establish school snack } \\
\text { programmes for vulnerable } \\
\text { Syrians and Jordanians } \\
\text { Engage Mol to expand health } \\
\text { education provision }\end{array}$ \\
\hline
\end{tabular}

Table A3.9: Summary table - child protection and psychosocial support

\begin{tabular}{|c|c|c|}
\hline $\begin{array}{l}\text { Challenges specific to child } \\
\text { protection and psychosocial } \\
\text { needs }\end{array}$ & $\begin{array}{l}\text { Effective practice addressing } \\
\text { needs }\end{array}$ & Remaining needed steps \\
\hline 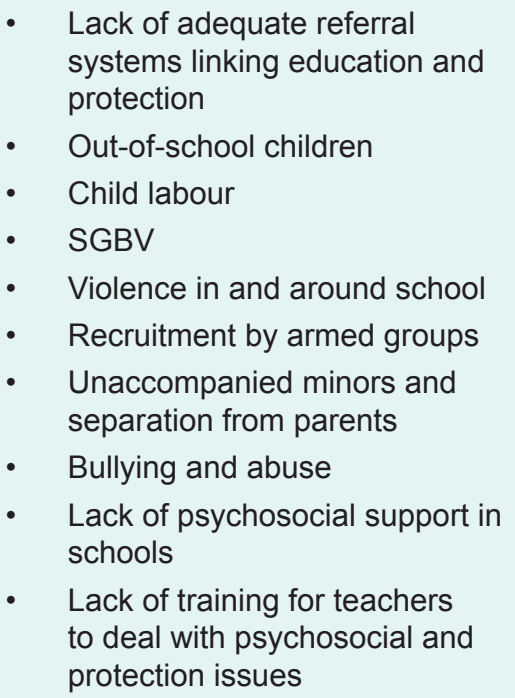 & $\begin{array}{l}\text { Focus on identifying need: } \\
\text { violence in schools survey and } \\
\text { work on case management } \\
\text { system } \\
\text { - } \quad \text { Makani centres integrate } \\
\text { protection and education } \\
\text { priorities } \\
\text { - Syrian assistant teachers } \\
\text { support classrooms and protect } \\
\text { children } \\
\text { National Ma'an and Tarbiyah } \\
\text { initiatives aim to change } \\
\text { attitudes }\end{array}$ & $\begin{array}{l}\text { - Invest in referral mechanisms } \\
\text { linking protection and education } \\
\text { Capacity building in schools } \\
\text { for psychosocial support: } \\
\text { teacher training and provision of } \\
\text { adequate school counsellors } \\
\text { - Expand Syrian assistant teacher } \\
\text { programme to provide protection } \\
\text { in, around and on the way to } \\
\text { schools } \\
\text { Establish coordinated strategy } \\
\text { across MOE schools to address } \\
\text { bullying and violence }\end{array}$ \\
\hline
\end{tabular}


Table A3.10: Summary table - WASH

\begin{tabular}{|c|c|c|}
\hline Challenges specific to WASH & $\begin{array}{l}\text { Effective practice addressing } \\
\text { needs }\end{array}$ & Remaining needed steps \\
\hline $\begin{array}{l}\text { - Continued infrastructural issues } \\
\text { in camp schools create safety } \\
\text { risks } \\
\text { Cleanliness issues limit use of } \\
\text { WASH facilities in schools } \\
\text { Health and hygiene concerns } \\
\text { lead to drop outs } \\
\text { Unreliable access to water } \\
\text { in refugee homes in host } \\
\text { communities may affect } \\
\text { attendance }\end{array}$ & $\begin{array}{l}\text { Sustainable investments in } \\
\text { capacity building: infrastructure, } \\
\text { WASH standards and expert } \\
\text { secondments to GOJ } \\
\text { Evidence based planning: needs } \\
\text { assessment in host community } \\
\text { schools } \\
\text { Gender-sensitive programming } \\
\text { and progress on gender- } \\
\text { appropriate facilities } \\
\text { WASH educational messages } \\
\text { delivered in formal and } \\
\text { alternative education settings }\end{array}$ & 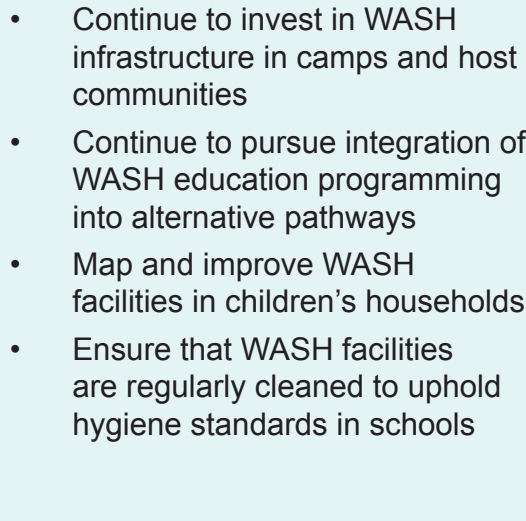 \\
\hline
\end{tabular}

Table A3.11: Summary table - sustainability

\section{Sustainability challenges}

- Political and strategic difficulty of acknowledging necessary trade-offs

- Incoherence of advocacy efforts and messaging among donor group and UN agencies

- Loss of institutional knowledge within UNICEF as a result of fast-paced staff turnover

- Building MOE capacity around management, infrastructure, quality assurance and staff salaries

- Engaging community voices in programming and ensuring buyin from Syrian and Jordanian parents

- Resource constraints and shortterm funding cycles, preventing longer-term strategic thinking at all levels of Response

\section{Effective practice addressing} needs

- UNICEF's added-value in advocating for EER needs with GOJ

- Major expansion of staffing and skills resources of UNICEF and partners

- Training provision for implementing partners

- Investments in implementing partner capacity and systems

- Engaging Syrians in programming and utilising existing skills through the Syrian assistant teachers initiative

- Inclusive education and spaces provided in alternative learning centres

\section{Remaining needed steps}

- $\quad$ Provide leadership to acknowledge necessary tradeoffs which must be made in Programme planning

- Utilise leadership role to establish dialogue and coordination among key influencers (UN agencies, donors) to ensure targeted advocacy efforts

- Ensure UNICEF internal management systems are adaptable enough to cope with high-paced change and staff turnover

- Invest in MOE to build management capability, pay teachers a sustainable wage, build infrastructure and expand quality assurance programmes

- Strengthen advocacy for longerterm thinking among the donor group, to lengthen funding cycles

- Increase investment in community empowerment, ensuring that beneficiary voices are incorporated at planning and implementation stages of all programming 
Table A3.12: Summary table - recommendations

\begin{tabular}{|c|c|c|}
\hline Recommendation & $\begin{array}{l}\text { Related DAC } \\
\text { Criteria }\end{array}$ & Supportive Findings \\
\hline $\begin{array}{l}\text { 1. Build upon } \\
\text { significant } \\
\text { accomplishments }\end{array}$ & $\begin{array}{ll}\text { - } & \text { Relevance } \\
\text { - } & \text { Effectiveness } \\
\text { - } & \text { Efficiency } \\
\text { - } & \text { Sustainability }\end{array}$ & $\begin{array}{l}\text { The MOE, UNICEF, implementing partners and donors have } \\
\text { enabled: structured formal education for } 130,000 \text { children, } \\
\text { alternative educational approaches for } 35,000 \text { children, } \\
\text { double-shifting of } 98 \text { additional schools to expand access, } \\
\text { training for 2,100 teachers, significant resource mobilisation, } \\
\text { and more. } \\
\text { Formal, non-formal and informal education has provided much } \\
\text { needed learning, structure, and purpose for children. }\end{array}$ \\
\hline $\begin{array}{l}\text { 2. Develop a clearly } \\
\text { articulated medium- } \\
\text { term } 5-10 \text { year } \\
\text { vision }\end{array}$ & $\begin{array}{ll}\text { - } & \text { Relevance } \\
\text { - } & \text { Effectiveness } \\
\text { - } & \text { Efficiency } \\
\text { - } & \text { Sustainability }\end{array}$ & $\begin{array}{l}\text { - Jordan and the international community will be responsible for } \\
\text { providing education for the Syrian refugees well into the future, } \\
\text { likely for a decade or more. } \\
\text { Neither key planning documents nor interviewees articulated } \\
\text { a clear medium-term vision capable of responding to these } \\
\text { serious challenges. } \\
\text { Short-term funding cycles and programmes are inefficient and } \\
\text { unable to sustainably address problems in the medium term. }\end{array}$ \\
\hline $\begin{array}{l}\text { 3. Develop a medium- } \\
\text { term outlook to } \\
\text { funding }\end{array}$ & $\begin{array}{ll}\text { - } & \text { Efficiency } \\
\text { - } & \text { Sustainability }\end{array}$ & $\begin{array}{l}\text { Funding for refugee education is currently dependent upon } \\
\text { resources from the Jordanian government and short-term } \\
\text { donor commitments. These will not be enough to support } \\
\text { medium-term needs for education access and quality. } \\
\text { - Meeting access and quality goals requires seeking medium- } \\
\text { term funding through other approaches, e.g. public private } \\
\text { partnerships or taxing Syrian labour in formal employment. } \\
\text { Lack of accountability systems prevents more direct funding to } \\
\text { the GOJ in support of public services like education. }\end{array}$ \\
\hline $\begin{array}{l}\text { 4. Prioritise expansion } \\
\text { of formal } \\
\text { education and } \\
\text { creation of school } \\
\text { spaces through } \\
\text { investing in school } \\
\text { infrastructure, } \\
\text { teacher salaries and } \\
\text { transportation }\end{array}$ & $\begin{array}{ll}\text { - } & \text { Relevance } \\
\text { - } & \text { Effectiveness } \\
\text { - } & \text { Sustainability }\end{array}$ & $\begin{array}{l}\text { - At least 97,000 Syrian children and 31,000 Jordanian children } \\
\text { are not attending formal school. } \\
\text { School space provision rests on school infrastructure, teachers } \\
\text { and supportive programmes. } \\
\text { Double shifts are the fastest way to expand infrastructure. } \\
\text { With only } 15 \text { per cent of Jordan's schools double-shifted, there } \\
\text { is room for expansion. Building prefabricated schools quickly } \\
\text { and cost-effectively has been a successful approach used by } \\
\text { UNICEF in Turkey. Renting and repurposing, transportation } \\
\text { and new infrastructure are other approaches. } \\
\text { Donors could cover salaries of } 1,000-2,000 \text { teachers for } \$ 6.8 \\
\text { million per year plus administration. } \\
\text { Supportive planning approaches of database building and } \\
\text { outreach are ongoing. }\end{array}$ \\
\hline $\begin{array}{l}\text { 5. Create formal } \\
\text { education at the } \\
\text { scale needed } \\
\text { for Syrian and } \\
\text { Jordanian children } \\
\text { who have missed } \\
\text { years of school }\end{array}$ & $\begin{array}{ll}\text { - } & \text { Effectiveness } \\
\text { - } & \text { Sustainability }\end{array}$ & $\begin{array}{l}\text { - An estimated } 65,000 \text { of the Syrian out-of-school children do } \\
\text { not qualify for formal education under MOE current policies, as } \\
\text { they have been out of school for longer than three years. An } \\
\text { additional 31,000 Jordanian children are out of school. } \\
\text { These children cannot be integrated into Jordanian } \\
\text { classrooms. Placing older children in younger classrooms is } \\
\text { demotivating and disruptive. } \\
\text { A formal, certified programme for these children in age- } \\
\text { appropriate clusters is needed, something similar to non- } \\
\text { formal education for younger children. This is needed at scale. }\end{array}$ \\
\hline
\end{tabular}




\begin{tabular}{|c|c|c|}
\hline Recommendation & $\begin{array}{l}\text { Related DAC } \\
\text { Criteria }\end{array}$ & Supportive Findings \\
\hline $\begin{array}{l}\text { 6reate a new } \\
\text { delivery model to } \\
\text { support short-term } \\
\text { rapid expansion } \\
\text { of education while } \\
\text { building long-term } \\
\text { sustainable options } \\
\text { for out-of-school } \\
\text { children }\end{array}$ & $\begin{array}{ll}\text { - } & \text { Relevance } \\
\text { - } & \text { Effectiveness } \\
\text { - } & \text { Efficiency } \\
\text { - } & \text { Sustainability }\end{array}$ & $\begin{array}{l}\text { The current management capacity of the MOE and UN } \\
\text { agencies for the education response is stretched to the limit, } \\
\text { and new management models are needed for the required } \\
\text { expansion. } \\
\text { Management model options include expansion of the MOE } \\
\text { system, development of UN-managed system like UNWRA } \\
\text { for the Palestinians, or a hybrid model that begins with UN } \\
\text { management and transitions to the government. } \\
\text { A hybrid model would have the advantage of being quick to } \\
\text { set up, while moving towards sustainable management by the } \\
\text { MOE in the medium term. }\end{array}$ \\
\hline $\begin{array}{l}\text { 7. Invest in MOE } \\
\text { capacity and } \\
\text { provide direct } \\
\text { operational support } \\
\text { to the MOE }\end{array}$ & $\begin{array}{ll}\text { - } & \text { Efficiency } \\
\text { - } & \text { Sustainability }\end{array}$ & $\begin{array}{l}\text { The MOE has only a few staff with responsibility for refugee } \\
\text { education. Resources are constrained, with the GOJ taking } \\
\text { loans to cover education and health costs for the refugees. } \\
\text { International investment in human capacity for managing the } \\
\text { crisis has been disproportionately focused towards salaries } \\
\text { and skills for UN agencies and NGO partners, and not for the } \\
\text { government. } \\
\text { It is important to enable the MOE to provide quality formal } \\
\text { education in the coming decade. The MOE has a foundation } \\
\text { of capacities in place, with a robust and expanding education } \\
\text { system. } \\
\text { While the MOE has school monitoring for first shifts, there is } \\
\text { a need to expand capacity and develop explicit guidance for } \\
\text { circumstances specific to refugees. } \\
\text { Accountability systems are a barrier to donor confidence to } \\
\text { direct funding of government public service provision. }\end{array}$ \\
\hline $\begin{array}{l}\text { 8. Improve the } \\
\text { performance of } \\
\text { double-shifted } \\
\text { schools to meet } \\
\text { specific needs of } \\
\text { both Jordanians } \\
\text { and Syrians }\end{array}$ & $\begin{array}{ll}\text { - } & \text { Effectiveness } \\
\text { - } & \text { Sustainability }\end{array}$ & $\begin{array}{l}\text { Double shifts in Jordan have reduced instructional time, } \\
\text { unequal resources for both shifts, and inexperienced and } \\
\text { lower-paid teachers in second shifts for Syrians. } \\
\text { Double shifts have been effective in providing quality } \\
\text { education in other countries when resources were similar } \\
\text { between shifts and when there was adequate instructional } \\
\text { time. } \\
\text { Using double shifts in Jordan for the Syrians has been } \\
\text { controversial because of concerns about unequal education } \\
\text { and social cohesion. } \\
\text { Some Syrian parents indicated separate shifts are better } \\
\text { suited to the needs of their overwhelmed children as they } \\
\text { adapt to new circumstances and a new curriculum. } \\
\text { Applying recommended guidelines for when and how to use } \\
\text { single shifts, double shifts, separation and integration could } \\
\text { solve some of the problems with quality and social cohesion. }\end{array}$ \\
\hline
\end{tabular}




\begin{tabular}{|c|c|c|}
\hline Recommendation & $\begin{array}{l}\text { Related DAC } \\
\text { Criteria }\end{array}$ & Supportive Findings \\
\hline $\begin{array}{l}\text { 9. Improve the } \\
\text { quality and safety } \\
\text { of the learning } \\
\text { environment in } \\
\text { MOE formal schools } \\
\text { in camps and host } \\
\text { communities }\end{array}$ & - Effectiveness & $\begin{array}{l}\text { - Findings from this evaluation highlight significant problems } \\
\text { with quality and safety in education: crowded classrooms; low } \\
\text { outcomes on the Jordanian Tawjihi test; teachers struggling } \\
\text { with a traumatised group of students; short instructional time } \\
\text { in double shifts; lack of pathways to further education and } \\
\text { adulthood; bullying; and lack of systematic psychosocial } \\
\text { support. } \\
\text { Quality of education has taken lower priority than access to } \\
\text { education during the early stages of the emergency. However, } \\
\text { improving the quality of education is now an agreed priority. } \\
\text { - Teacher quality could be supported by: reducing class sizes, } \\
\text { school monitoring, mixing experienced and inexperienced } \\
\text { teachers, paying teachers adequately and training. } \\
\text { - Syrian teachers are an underutilised resource. } \\
\text { Curriculum standards and pathways between alternative } \\
\text { education, formal education, higher education and } \\
\text { employment are lacking. } \\
\text { Psychosocial support was not provided in a systematic way } \\
\text { across host communities and camps. } \\
\text { Bullying is a significant problem in schools, and there are no } \\
\text { systematic approaches to address it. } \\
\text { The EER is poor at including the voices of the Syrians } \\
\text { themselves in education. } \\
\text { Classrooms are in need of repairs. }\end{array}$ \\
\hline $\begin{array}{l}\text { 10. Improve access } \\
\text { and quality by } \\
\text { addressing social } \\
\text { challenges and } \\
\text { targeting the } \\
\text { different needs of } \\
\text { girls and boys }\end{array}$ & $\begin{array}{ll}\text { - } & \text { Effectiveness } \\
\text { - } & \text { Sustainability }\end{array}$ & $\begin{array}{l}\text { - This evaluation found that access is related to multiple } \\
\text { complex factors involving the provision of education, the } \\
\text { location of the child and the characteristics of the family. } \\
\text { Girls and boys face distinct gendered challenges that require } \\
\text { targeted responses, both in the classroom and outside. Boys } \\
\text { have lower access to formal education at all levels, and have } \\
\text { greater levels of child labour. Girls experience high levels of } \\
\text { early marriage, and families' concerns about safety limits girls' } \\
\text { transportation and mobility. } \\
\text { While schools and implementing partners have received } \\
\text { gender sensitivity training, and cross-sectoral coordination } \\
\text { is facilitated by gender focus points, there is still a need to } \\
\text { address gaps in gender sensitive programming. Existing } \\
\text { approaches have been limited. }\end{array}$ \\
\hline $\begin{array}{l}\text { 11. Prioritise } \\
\text { programmes and } \\
\text { spending based on } \\
\text { options analyses }\end{array}$ & - Efficiency & $\begin{array}{l}\text { Education planning lacks explicit, detailed analysis of costs, } \\
\text { benefits, priorities and outcomes. There is no systematic } \\
\text { approach to options appraisals. } \\
\text { Long and costly lists of programmes that lack a medium-term } \\
\text { vision, supporting priorities and implementation plans have } \\
\text { been described as 'wish lists'. } \\
\text { Resources are too limited to cover all of the desired } \\
\text { programmes. }\end{array}$ \\
\hline
\end{tabular}


Recommendation

12. Strengthen sectoral coordination and management

13. Ensure flexibility and adaptability as circumstances change

Relevance

- Effectiveness

- Efficiency

- Sustainability

14. Develop a phased - Relevance implementation plan . Effectiveness with 'quick wins'

\section{Supportive Findings}

- There exists an effective and improving coordination system which should be strengthened.

- $\quad$ ESWG is underpowered for the task, and there are weak mechanisms to maximise synergies, reduce duplication, secure economies of scale and share infrastructure; to share infrastructural, training and other HR costs; to coordinate procurement, scale up effective practice, share data collection and pool capacities toward shared outcomes.

- Vital unknowns affect planning, requiring adaption of the education strategy over time.

- Approaches such as scenario planning and maturity models have been effective in other situations of planning under conditions of uncertainty.

- Among many recommendations, steps are needed to have impact for many children quickly.

- A prioritised, costed, optioned implementation plan is needed. 


\section{Annex 4: Comparison (where possible) of budgeting requirements between $3 R P$ and RRP6}

\begin{tabular}{|c|c|c|}
\hline & $\begin{array}{l}\text { Refugee response and resilience } \\
\text { response requirements (3RP) }\end{array}$ & RRP6 \\
\hline $\begin{array}{l}\text { Early childhood development in } \\
\text { vulnerable communities }\end{array}$ & $\$ 5,783,750$ & $\$ 1,220,520$ \\
\hline Teacher training programme & $\$ 6,295,000$ & $\$ 1,115,081$ \\
\hline $\begin{array}{l}\text { Alternative certified education } \\
\text { pathways project }\end{array}$ & $\$ 22,396,000$ & $\$ 12,129,727$ \\
\hline Violence-free schools & $\$ 346,600$ & \\
\hline $\begin{array}{l}\text { Equal access to education } \\
\text { opportunities for Jordanians }\end{array}$ & $\$ 41,579,096$ & $\begin{array}{l}\$ 8,970,035 \text { Jordanian public } \\
\text { schools supported with additional } \\
\text { learning spaces }\end{array}$ \\
\hline Expansion of higher education & $\$ 57,250,000$ & $\begin{array}{l}\$ 5,950,800 \text { Youth benefit from } \\
\text { secondary and higher education }\end{array}$ \\
\hline $\begin{array}{l}\text { MOE enhanced institutional capacity } \\
\text { project }\end{array}$ & $\$ 650,000$ & \\
\hline Formal education & & $\$ 12,137,292$ \\
\hline $\begin{array}{l}\text { Children with specific needs access } \\
\text { educational and psychosocial } \\
\text { services }\end{array}$ & & $\$ 1,206,600$ \\
\hline $\begin{array}{l}\text { Essential learning materials } \\
\text { including basic stationery and other } \\
\text { education supplies }\end{array}$ & & $\$ 3,840,211$ \\
\hline
\end{tabular}

